\begin{tabular}{|c|l|}
\hline Title & Lightlike hy persurfaces al ong spacelike submanifolds in anti-de Sitter space \\
\hline Author(s) & Izumiya, Shyuichi \\
\hline Citation & Journal of mathematical physics, 56(11), 112502 \\
\hline https:/doi.org/10.1063/L.4936148 \\
\hline Issue Date & 2015-11-25 \\
\hline Doc URL & http://hdl.handle.net/2115/60523 \\
\hline Type & article \\
\hline File Information & 1.4936148.pdf \\
\hline
\end{tabular}

Instructions for use 


\title{
Lightlike hypersurfaces along spacelike submanifolds in anti-de Sitter space
}

\author{
Shyuichi Izumiyaa) \\ Department of Mathematics, Hokkaido University, Sapporo 060-0810, Japan
}

(Received 7 March 2015; accepted 8 November 2015; published online 25 November 2015)

\begin{abstract}
Anti-de Sitter space is the Lorentzian space form with negative curvature. In this paper, we consider lightlike hypersurfaces along spacelike submanifolds in antide Sitter space with general codimension. In particular, we investigate the singularities of lightlike hypersurfaces as an application of the theory of Legendrian singularities. (C) 2015 AIP Publishing LLC. [http://dx.doi.org/10.1063/1.4936148]
\end{abstract}

\section{INTRODUCTION}

Anti-de Sitter (AdS) space is one of the Lorentz space forms which has rich geometric properties. It is defined as a pseudo-sphere with negative curvature in semi-Euclidean space with index 2 which admits the biggest symmetry in Riemannian or Lorentz space forms. Anti-de Sitter space can be naturally considered as a Lorentzian version (generalization) of hyperbolic space. Recently, we discovered interesting geometric properties of submanifolds in hyperbolic space as an application of the theory of Legendrian/Lagrangian singularities. ${ }^{13,15,14,12}$ Therefore, anti-de Sitter space might have rich geometric properties comparing with hyperbolic space. This is one of the motivations for the investigation of submanifolds in anti-de Sitter space from a mathematical viewpoint.

On the other hand, anti-de Sitter space plays important roles in theoretical physics such as the theory of general relativity, the string theory, and the brane world scenario. ${ }^{2,22,28,31}$ It is well known that Lorentzian space forms are classified into three types depending on the value of the scalar curvature. One of them is Lorentz-Minkowski space which has zero curvature. The Lorentz space form with positive curvature is de Sitter space. Anti-de Sitter space is a Lorentzian space form with negative curvature. Recently, submanifolds in Lorentz-Minkowski space or de Sitter space have been well investigated (cf. Refs. 10, 8, 16, 9, 19, and 20). However, there are not so many results on submanifolds in anti-de Sitter space, in particular from the viewpoint of singularity theory. ${ }^{11}$ The lightlike hypersurfaces (i.e., the light-sheets in physics) are important objects in theoretical physics because they provide good models for different types of horizons. ${ }^{4,25}$ A lightlike hypersurface is generally a ruled hypersurface along a spacelike submanifold with codimension two whose rulings are lightlike geodesics. In this paper, we consider lightlike hypersurfaces along spacelike submanifolds with general codimension in anti-de Sitter space. Moreover, the lightlike hypersurface in anti-de Sitter 5-space is a very important subject in the brane world scenario. ${ }^{17,2,28}$

In the meantime, tools in the theory of singularities have proven to be useful descriptions of geometrical properties of submanifolds immersed in different ambient spaces, from both the local and global viewpoints. ${ }^{13,10,12,16,9,21}$ The natural connection between geometry and singularities relies on the basic fact that the contacts of a submanifold with the models of the ambient space can be described by means of the analysis of the singularities of appropriate families of contact functions, or equivalently, of their associated Legendrian maps. ${ }^{1,26,32}$ When working in a Lorentz space form, the properties associated with the contacts of a given submanifold with lightcones have a special relevance. In Refs. 10, 16, and 18, a framework was constructed for the study of spacelike submanifolds with codimension two in Lorentz-Minkowski space or de Sitter space and a Lorentz invariant concerning their contacts with models related to lightlike hyperplanes was discovered. The geometry described in this framework is called the lightlike geometry of spacelike submanifolds

a)e-mail: izumiya@math.sci.hokudai.ac.jp 
with codimension two. By using the invariants of lightlike geometry, the singularities of lightlike hypersurfaces along spacelike submanifolds with codimension two in Lorentz-Minkowski space or de Sitter space were investigated in Refs. 8, 7, and 18. However, the situation is rather complicated for the general codimensional case. The main difference from the Euclidean space (or, hyperbolic space) case is the fiber of the canal hypersurface of a spacelike submanifold is neither connected nor compact. In order to avoid the above difficulty, we arbitrarily choose a timelike future directed unit normal vector field along the spacelike submanifold which always exists for an orientable manifold (cf. Sec. III). Then, we construct the unit spherical normal bundle relative to the above timelike unit normal vector field, which can be considered as a codimension two spacelike canal submanifold of the ambient space. Therefore, we can apply the idea of the lightlike geometry of spacelike submanifolds of the ambient space with codimension two. In this paper, we apply the idea of this framework and the theory of Legendrian singularities to investigate the singularities of lightlike hypersurfaces along spacelike submanifolds in anti-de Sitter space with general codimension.

In Sec. III, we construct the framework of the lightlike geometry of spacelike submanifolds with general codimension analogous to Ref. 12. The notion of lightlike hypersurfaces along spacelike submanifolds is introduced and the basic properties are investigated in Sec. IV. The notion of the anti-de Sitter height functions families is useful for the study of lightlike hypersurfaces (cf. Sec. IV). The critical value set of the lightlike hypersurface along a spacelike submanifold is called the lightlike focal set of the submanifold. In Sec. V, we show that the lightlike focal set of a spacelike submanifold is a point if and only if the lightlike hypersurface along the submanifold is a subset of a lightcone (Proposition 5.1). Therefore, an anti-de Sitter lightcone is a model hypersurface of lightlike hypersurfaces. The geometric meaning of the singularities of the lightlike hypersurface is described by the theory of contact of submanifolds with model hypersurfaces. Moreover, as an application of the theory of Legendrian singularities, we show that two lightlike hypersurfaces are locally diffeomorphic if and only if the types of the contact of spacelike submanifolds with lightcones are the same in the sense of Montaldi $^{26}$ under some generic conditions (Theorem 5.5). In Sec. VI, we describe the case of codimension two as a special case. We describe the detailed properties of lightlike focal sets of spacelike surfaces in anti-de Sitter 4-space. We also investigate spacelike curves in anti-de Sitter 4-space as the simplest case of a higher codimension in Sec. VII.

We shall assume throughout the whole paper that all the maps and manifolds are $C^{\infty}$ unless the contrary is explicitly stated.

\section{BASIC FACTS AND NOTATIONS ON SEMI-EUCLIDEAN SPACE WITH INDEX 2}

In this section, we prepare the basic notions on semi-Euclidean $(\mathrm{n}+2)$-space with index 2 . For details of semi-Euclidean geometry, see Ref. 27. Let $\mathbb{R}^{n+2}=\left\{\left(x_{-1}, x_{0}, x_{1}, \ldots, x_{n}\right) \mid x_{i} \in \mathbb{R}(i=\right.$ $-1,0, \ldots, n)\}$ be an $(\mathrm{n}+2)$-dimensional vector space. For any vectors $\boldsymbol{x}=\left(x_{-1}, x_{0}, x_{1}, \ldots, x_{n}\right)$ and $\boldsymbol{y}=\left(y_{-1}, y_{0}, y_{1}, \ldots, y_{n}\right)$ in $\mathbb{R}^{n+2}$, the pseudo-scalar product of $\boldsymbol{x}$ and $\boldsymbol{y}$ is defined to be $\langle\boldsymbol{x}, \boldsymbol{y}\rangle=$ $-x_{-1} y_{-1}-x_{0} y_{0}+\sum_{i=1}^{n} x_{i} y_{i}$. We call $\left(\mathbb{R}^{n+2},\langle\rangle,\right)$ semi-Euclidean $(\mathrm{n}+2)$-space with index 2 and write $\mathbb{R}_{2}^{n+2}$ instead of $\left(\mathbb{R}^{n+2},\langle\rangle,\right)$. We say that a non-zero vector $\boldsymbol{x}$ in $\mathbb{R}_{2}^{n+2}$ is spacelike, null, or timelike if $\langle\boldsymbol{x}, \boldsymbol{x}\rangle>0,\langle\boldsymbol{x}, \boldsymbol{x}\rangle=0$, or $\langle\boldsymbol{x}, \boldsymbol{x}\rangle<0$, respectively. The norm of the vector $\boldsymbol{x} \in \mathbb{R}_{2}^{n+2}$ is defined to be $\|x\|=\sqrt{|\langle\boldsymbol{x}, \boldsymbol{x}\rangle|}$. We define the signature of $\boldsymbol{x}$ by

$$
\operatorname{sign}(\boldsymbol{x})= \begin{cases}1 & \boldsymbol{x} \text { is spacelike } \\ 0 & \boldsymbol{x} \text { is null, } \\ -1 & \boldsymbol{x} \text { is timelike }\end{cases}
$$

For a non-zero vector $\boldsymbol{n} \in \mathbb{R}_{2}^{n+2}$ and a real number $c$, we define the hyperplane with pseudo-normal $n$ by

$$
H P(\boldsymbol{n}, c)=\left\{\boldsymbol{x} \in \mathbb{R}_{2}^{n+2} \mid\langle\boldsymbol{x}, \boldsymbol{n}\rangle=c\right\} .
$$

We call $H P(\boldsymbol{n}, c)$ a Lorentz hyperplane, a semi-Euclidean hyperplane with index 2 or a null hyperplane if $\boldsymbol{n}$ is timelike, spacelike, or null, respectively. 
We now define an anti-de Sitter $(n+1)$-space (briefly, $A d S(n+1)$-space) by

$$
A d S^{n+1}=\left\{\boldsymbol{x} \in \mathbb{R}_{2}^{n+2} \mid\langle\boldsymbol{x}, \boldsymbol{x}\rangle=-1\right\}=H_{1}^{n+1},
$$

a unit pseudo-( $n+1)$-sphere with index 2 by

$$
S_{2}^{n+1}=\left\{\boldsymbol{x} \in \mathbb{R}_{2}^{n+2} \mid\langle\boldsymbol{x}, \boldsymbol{x}\rangle=1\right\}
$$

and a (closed) nullcone with vertex $\boldsymbol{a}$ by

$$
\Lambda_{a}^{n+1}=\left\{\boldsymbol{x} \in \mathbb{R}_{2}^{n+2} \mid\langle\boldsymbol{x}-\boldsymbol{a}, \boldsymbol{x}-\boldsymbol{a}\rangle=0\right\} .
$$

In particular, we denote that $\Lambda^{*}=\Lambda_{0}^{n+1} \backslash\{\boldsymbol{0}\}$ and also call it the (open) nullcone. Our main subject in this paper is $A d S^{n+1}$. Since there are timelike closed curves in $A d S^{n+1}$, the causality of $A d S^{n+1}$ is violated. In order to avoid such a situation, it is usually considered the universal covering space $\widetilde{A d S}^{n+1}$ of $A d S^{n+1}$ in physics which is called the universal anti-de Sitter space. We remark that the local structure of these spaces are the same.

For any $\boldsymbol{x}_{1}, \ldots, \boldsymbol{x}_{n} \in \mathbb{R}_{2}^{n+2}$. We define a vector $\boldsymbol{x}_{1} \wedge \cdots \wedge \boldsymbol{x}_{n}$ by

$$
\boldsymbol{x}_{1} \wedge \cdots \wedge \boldsymbol{x}_{n}=\left|\begin{array}{ccccc}
-\boldsymbol{e}_{-1} & -\boldsymbol{e}_{0} & \boldsymbol{e}_{1} & \cdots & \boldsymbol{e}_{n} \\
x_{-1}^{1} & x_{0}^{1} & x_{1}^{1} & \cdots & x_{n}^{1} \\
\vdots & \vdots & \vdots & \vdots & \vdots \\
x_{-1}^{n} & x_{0}^{n} & x_{1}^{n} & \cdots & x_{n}^{n}
\end{array}\right|,
$$

where $\left\{\boldsymbol{e}_{-1}, \boldsymbol{e}_{0}, \boldsymbol{e}_{1}, \ldots, \boldsymbol{e}_{n}\right\}$ is the canonical basis of $\mathbb{R}_{2}^{n+2}$ and $\boldsymbol{x}_{i}=\left(x_{-1}^{i}, x_{0}^{i}, x_{1}^{i}, \ldots, x_{n}^{i}\right)$. We can easily check that

$$
\left\langle\boldsymbol{x}, \boldsymbol{x}_{1} \wedge \cdots \wedge \boldsymbol{x}_{n}\right\rangle=\operatorname{det}\left(\boldsymbol{x}, \boldsymbol{x}_{1}, \ldots, \boldsymbol{x}_{n}\right),
$$

so that $\boldsymbol{x}_{1} \wedge \cdots \wedge \boldsymbol{x}_{n}$ is pseudo-orthogonal to any $\boldsymbol{x}_{i}$ (for $i=1, \ldots, n$ ).

\section{SPACELIKE SUBMANIFOLDS IN ANTI-DE SITTER SPACE}

We introduce in this section the basic geometrical tools for the study of spacelike submanifolds in the anti-de Sitter $(n+1)$-space.

Consider the orientation of $\mathbb{R}_{2}^{n+2}$ provided by the condition that $\operatorname{det}\left(\boldsymbol{e}_{-1}, \boldsymbol{e}_{0}, \boldsymbol{e}_{1}, \ldots, \boldsymbol{e}_{n}\right)>0$. This orientation induces the orientation of $x_{-1} x_{0}$-plane, so that it gives a time orientation on $A d S^{n+1}$. If we consider the universal anti-de Sitter space $\widetilde{A d S}^{n+1}$, we can determine the future direction.

We consider a spacelike embedding $X: U \rightarrow A d S^{n+1}$ from an open subset $U \subset \mathbb{R}^{s}$ with $s+k=$ $n+1$. We write $M=\boldsymbol{X}(U)$ and identify $M$ and $U$ through the embedding $\boldsymbol{X}$. We say that $\boldsymbol{X}$ is spacelike if the tangent space $T_{p} M$ consists only of spacelike vectors (i.e., spacelike subspace) for any point $p \in M$. In this case, the pseudo-normal space $N_{p}(M)$ in $\mathbb{R}_{2}^{n+2}$ is a $k+1$-dimensional semi-Euclidean space with index 2 (cf. Ref. 27). We write $N(M)$ as the pseudo-normal bundle in $\mathbb{R}_{2}^{n+2}$ over $M$. On the pseudo-normal space $N_{p}(M)$, we have two kinds of pseudo-spheres

$$
\begin{aligned}
N_{p}(M ;-1) & =\left\{\boldsymbol{v} \in N_{p}(M) \mid\langle\boldsymbol{v}, \boldsymbol{v}\rangle=-1\right\}, \\
N_{p}(M ; 1) & =\left\{\boldsymbol{v} \in N_{p}(M) \mid\langle\boldsymbol{v}, \boldsymbol{v}\rangle=1\right\},
\end{aligned}
$$

so that we have two unit spherical normal bundles over $M$,

$$
N(M ;-1)=\bigcup_{p \in M} N_{p}(M ;-1) \text { and } N(M ; 1)=\bigcup_{p \in M} N_{p}(M ; 1) .
$$

Then, we have the Whitney sum decomposition

$$
T \mathbb{R}_{2}^{n+2} \mid M=T M \oplus N(M) .
$$

By definition, $\boldsymbol{X}(u)$ is one of the timelike unit normal vectors of $M$ at $p=\boldsymbol{X}(u)$, so that $\boldsymbol{X} \in N_{p}(M)$. Since $A d S^{n+1}$ is time oriented, we can arbitrarily choose an adopted unit timelike normal section $\boldsymbol{n}^{T}(u) \in N_{p}(M)$ pseudo-orthogonal to $\boldsymbol{X}(u)$ even globally. Here, we say that $\boldsymbol{n}^{T}$ is adopted if

$$
\operatorname{det}\left(\boldsymbol{X}(u), \boldsymbol{n}^{T}(u), \boldsymbol{e}_{1}, \ldots, \boldsymbol{e}_{n}\right)>0 .
$$


Therefore, we have the pseudo-orthonormal complement $\left(\left\langle\boldsymbol{X}(u), \boldsymbol{n}^{T}(u)\right\rangle_{\mathbb{R}}\right)^{\perp}$ in $N_{p}(M)$ which is a $(k-1)$-dimensional subspace of $N_{p}(M)$. We define a $(k-2)$-dimensional spacelike unit sphere in $N_{p}(M)$ by

$$
N_{1}(M)_{p}\left[\boldsymbol{n}^{T}\right]=\left\{\boldsymbol{\xi} \in N_{p}(M ; 1) \mid\left\langle\boldsymbol{\xi}, \boldsymbol{n}^{T}(p)\right\rangle=\langle\boldsymbol{\xi}, \boldsymbol{X}(u)\rangle=0, p=\boldsymbol{X}(u)\right\} .
$$

Then, we have a spacelike unit $k$-2-spherical bundle over $M$ with respect to $\boldsymbol{n}^{T}$ defined by

$$
N_{1}(M)\left[\boldsymbol{n}^{T}\right]=\bigcup_{p \in M} N_{1}(M)_{p}\left[\boldsymbol{n}^{T}\right] .
$$

Since we have $T_{(p, \xi)} N_{1}(M)\left[\boldsymbol{n}^{T}\right]=T_{p} M \times T_{\xi} N_{1}(M)_{p}\left[\boldsymbol{n}^{T}\right]$, we have the canonical Riemannian metric on $N_{1}(M)\left[\boldsymbol{n}^{T}\right]$. We denote the Riemannian metric on $N_{1}(M)\left[\boldsymbol{n}^{T}\right]$ by $\left(G_{i j}(p, \boldsymbol{\xi})\right)_{1 \leqslant i, j \leqslant n-1}$. We now arbitrarily choose (at least locally) a unit spacelike normal vector field $\boldsymbol{n}^{S}$, with $\boldsymbol{n}^{S}(u) \in N_{1}(M)_{p}\left[\boldsymbol{n}^{T}\right]$, where $p=\boldsymbol{X}(u)$. We call $\left(\boldsymbol{n}^{T}, \boldsymbol{n}^{S}\right)$ an adopted pair of normal vector fields along $M$. Clearly, the vectors $\boldsymbol{n}^{T}(u) \pm \boldsymbol{n}^{S}(u)$ are null. We define a mapping

$$
\mathbb{N G}\left(\boldsymbol{n}^{T}, \boldsymbol{n}^{S}\right): U \longrightarrow \Lambda^{*}
$$

by $\mathbb{N G}\left(\boldsymbol{n}^{T}, \boldsymbol{n}^{S}\right)(u)=\boldsymbol{n}^{T}(u)+\boldsymbol{n}^{S}(u)$. We call it the nullcone Gauss image of $M=\boldsymbol{X}(U)$ with respect to $\left(\boldsymbol{n}^{T}, \boldsymbol{n}^{S}\right)$. With the identification of $M$ and $U$ through $X$, we have the linear mapping provided by the derivative of the nullcone Gauss image $\mathbb{N} G\left(\boldsymbol{n}^{T}, \boldsymbol{n}^{S}\right)$ at each point $p \in M$ as follows:

$$
d_{p} \mathbb{N G}\left(\boldsymbol{n}^{T}, \boldsymbol{n}^{S}\right): T_{p} M \longrightarrow T_{p} \mathbb{R}_{1}^{n+1}=T_{p} M \oplus N_{p}(M) .
$$

Consider the orthogonal projections $\pi^{t}: T_{p} M \oplus N_{p}(M) \rightarrow T_{p}(M)$. We define

$$
d_{p} \mathbb{N} G\left(n^{T}, n^{S}\right)^{t}=\pi^{t} \circ d_{p}\left(\boldsymbol{n}^{T}+\boldsymbol{n}^{S}\right) .
$$

We call the linear transformation $S_{p}\left(\boldsymbol{n}^{T}, \boldsymbol{n}^{S}\right)=-d_{p} \mathbb{N} G\left(\boldsymbol{n}^{T}, \boldsymbol{n}^{S}\right)^{t}$ the $\left(\boldsymbol{n}^{T}, \boldsymbol{n}^{S}\right)$-shape operator of $M=$ $\boldsymbol{X}(U)$ at $p=\boldsymbol{X}(u)$. Let $\left\{\kappa_{i}\left(\boldsymbol{n}^{T}, \boldsymbol{n}^{S}\right)(p)\right\}_{i=1}^{s}$ be the eigenvalues of $S_{p}\left(\boldsymbol{n}^{T}, \boldsymbol{n}^{S}\right)$, which are called the nullcone principal curvatures with respect to $\left(\boldsymbol{n}^{T}, n^{S}\right)$ at $p=X(u)$. Then, the nullcone Gauss-Kronecker curvature with respect to $\left(\boldsymbol{n}^{T}, \boldsymbol{n}^{S}\right)$ at $p=\boldsymbol{X}(u)$ is defined by

$$
K_{N}\left(\boldsymbol{n}^{T}, \boldsymbol{n}^{S}\right)(p)=\operatorname{det} S_{p}\left(\boldsymbol{n}^{T}, \boldsymbol{n}^{S}\right) .
$$

We say that a point $p=\boldsymbol{X}(u)$ is an $\left(\boldsymbol{n}^{T}, \boldsymbol{n}^{S}\right)$-umbilical point if

$$
S_{p}\left(\boldsymbol{n}^{T}, \boldsymbol{n}^{S}\right)=\kappa\left(\boldsymbol{n}^{T}, \boldsymbol{n}^{S}\right)(p) 1_{T_{p} M} .
$$

We say that $M=\boldsymbol{X}(U)$ is totally $\left(\boldsymbol{n}^{T}, \boldsymbol{n}^{S}\right)$-umbilical if all points on $M$ are $\left(\boldsymbol{n}^{T}, \boldsymbol{n}^{S}\right)$-umbilical. Moreover, $M=\boldsymbol{X}(U)$ is said to be totally nullcone umbilical if it is totally $\left(\boldsymbol{n}^{T}, \boldsymbol{n}^{S}\right)$-umbilical for any adopted pair $\left(\boldsymbol{n}^{T}, \boldsymbol{n}^{S}\right)$.

We deduce now the nullcone Weingarten formula. Since $\boldsymbol{X}_{u_{i}}(i=1, \ldots, s)$ are spacelike vectors, we have a Riemannian metric (the first fundamental form) on $M=\boldsymbol{X}(U)$ defined by $d s^{2}=$ $\sum_{i=1}^{s} g_{i j} d u_{i} d u_{j}$, where $g_{i j}(u)=\left\langle\boldsymbol{X}_{u_{i}}(u), \boldsymbol{X}_{u_{j}}(u)\right\rangle$ for any $u \in U$. We also have the nullcone second fundamental invariant with respect to the normal vector field $\left(\boldsymbol{n}^{T}, \boldsymbol{n}^{S}\right)$ defined by $h_{i j}\left(\boldsymbol{n}^{T}, \boldsymbol{n}^{S}\right)(u)=$ $\left\langle-\left(\boldsymbol{n}^{T}+\boldsymbol{n}^{S}\right)_{u_{i}}(u), \boldsymbol{X}_{u_{j}}(u)\right\rangle$ for any $u \in U$. By similar arguments to those in the proof of Ref. 16, Proposition 3.2, we have the following proposition.

Proposition 3.1. We choose a pseudo-orthonormal frame $\left\{\boldsymbol{X}, \boldsymbol{n}^{T}, \boldsymbol{n}_{1}^{S}, \ldots, \boldsymbol{n}_{k-1}^{S}\right\}$ of $N(M)$, with $\boldsymbol{n}_{k-1}^{S}=\boldsymbol{n}^{S}$. Then, we have the following nullcone Weingarten formula:

(a) $\mathbb{N} G\left(\boldsymbol{n}^{T}, \boldsymbol{n}^{S}\right)_{u_{i}}=\left\langle\boldsymbol{n}_{u_{i}}^{T}, \boldsymbol{n}^{S}\right\rangle\left(\boldsymbol{n}^{T}+\boldsymbol{n}^{S}\right)+\sum_{\ell=1}^{k-2}\left\langle\left(\boldsymbol{n}^{T}+\boldsymbol{n}^{S}\right)_{u_{i}}, \boldsymbol{n}_{\ell}^{S}\right\rangle \boldsymbol{n}_{\ell}^{S}-\sum_{j=1}^{s} h_{i}^{j}\left(\boldsymbol{n}^{T}, \boldsymbol{n}^{S}\right) \boldsymbol{X}_{u_{j}}$.

(b) $\pi^{t} \circ \mathbb{N} G\left(n^{T}, n^{S}\right)_{u_{i}}=-\sum_{j=1}^{s} h_{i}^{j}\left(\boldsymbol{n}^{T}, \boldsymbol{n}^{S}\right) \boldsymbol{X}_{u_{j}}$.

Here, $\left(h_{i}^{j}\left(\boldsymbol{n}^{T}, \boldsymbol{n}^{S}\right)\right)=\left(h_{i k}\left(\boldsymbol{n}^{T}, \boldsymbol{n}^{S}\right)\right)\left(g^{k j}\right)$ and $\left(g^{k j}\right)=\left(g_{k j}\right)^{-1}$.

As a consequence of the above proposition, we have an explicit expression of the nullcone curvature by

$$
K_{N}\left(\boldsymbol{n}^{T}, \boldsymbol{n}^{S}\right)=\frac{\operatorname{det}\left(h_{i j}\left(\boldsymbol{n}^{T}, \boldsymbol{n}^{S}\right)\right)}{\operatorname{det}\left(g_{\alpha \beta}\right)} .
$$


Since $\left\langle-\left(\boldsymbol{n}^{T}+\boldsymbol{n}^{S}\right)(u), X_{u_{j}}(u)\right\rangle=0$, we have $h_{i j}\left(\boldsymbol{n}^{T}, \boldsymbol{n}^{S}\right)(u)=\left\langle\boldsymbol{n}^{T}(u)+\boldsymbol{n}^{S}(u), X_{u_{i} u_{j}}(u)\right\rangle$. Therefore, the nullcone second fundamental invariant at a point $p_{0}=\boldsymbol{X}\left(u_{0}\right)$ depends only on the values $\boldsymbol{n}^{T}\left(u_{0}\right)+\boldsymbol{n}^{S}\left(u_{0}\right)$ and $\boldsymbol{X}_{u_{i} u_{j}}\left(u_{0}\right)$, respectively. Thus, the nullcone curvatures also depend only on $\boldsymbol{n}^{T}\left(u_{0}\right)+\boldsymbol{n}^{S}\left(u_{0}\right), \boldsymbol{X}_{u_{i}}\left(u_{0}\right)$, and $\boldsymbol{X}_{u_{i} u_{j}}\left(u_{0}\right)$, which are independent of the derivations of the vector fields $\boldsymbol{n}^{T}$ and $\boldsymbol{n}^{S}$. We write $\kappa_{i}\left(\boldsymbol{n}_{0}^{T}, \boldsymbol{n}_{0}^{S}\right)\left(p_{0}\right)(i=1, \ldots, s)$ and $K_{N}\left(\boldsymbol{n}_{0}^{T}, \boldsymbol{n}_{0}^{S}\right)\left(u_{0}\right)$ as the nullcone curvatures at $p_{0}=\boldsymbol{X}\left(u_{0}\right)$ with respect to $\left(\boldsymbol{n}_{0}^{T}, \boldsymbol{n}_{0}^{S}\right)=\left(\boldsymbol{n}^{T}\left(u_{0}\right), \boldsymbol{n}^{S}\left(u_{0}\right)\right)$. We might also say that a point $p_{0}=\boldsymbol{X}\left(u_{0}\right)$ is $\left(\boldsymbol{n}_{0}^{T}, \boldsymbol{n}_{0}^{S}\right)$-umbilical because the nullcone $\left(\boldsymbol{n}^{T}, \boldsymbol{n}^{S}\right)$-shape operator at $p_{0}$ depends only on the normal vectors $\left(\boldsymbol{n}_{0}^{T}, \boldsymbol{n}_{0}^{S}\right)$. So, we denote that $h_{i j}\left(\boldsymbol{n}^{T}, \boldsymbol{\xi}\right)\left(u_{0}\right)=h_{i j}\left(\boldsymbol{n}^{T}, \boldsymbol{n}^{S}\right)\left(u_{0}\right)$ and $K_{N}\left(\boldsymbol{n}^{T}, \boldsymbol{\xi}\right)\left(p_{0}\right)=$ $K_{N}\left(\boldsymbol{n}_{0}^{T}, \boldsymbol{n}_{0}^{S}\right)\left(p_{0}\right)$, where $\boldsymbol{\xi}=\boldsymbol{n}^{S}\left(u_{0}\right)$ for some local extension $\boldsymbol{n}^{T}(u)$ of $\boldsymbol{\xi}$. Analogously, we say that a point $p_{0}=\boldsymbol{X}\left(u_{0}\right)$ is an $\left(\boldsymbol{n}_{0}^{T}, \boldsymbol{n}_{0}^{S}\right)$-parabolic point of $\boldsymbol{X}: U \longrightarrow \mathbb{R}_{1}^{n+1}$ if $K_{N}\left(\boldsymbol{n}_{0}^{T}, \boldsymbol{n}_{0}^{S}\right)\left(u_{0}\right)=0$. We also say that a point $p_{0}=\boldsymbol{X}\left(u_{0}\right)$ is a $\left(\boldsymbol{n}_{0}^{T}, \boldsymbol{n}_{0}^{S}\right)$-flat point if it is an $\left(\boldsymbol{n}_{0}^{T}, \boldsymbol{n}_{0}^{S}\right)$-umbilical point and $K_{N}\left(\boldsymbol{n}_{0}^{T}, \boldsymbol{n}_{0}^{S}\right)\left(u_{0}\right)=0$.

On the other hand, we define a map $\mathbb{N} G\left(\boldsymbol{n}^{T}\right): N_{1}(M)\left[\boldsymbol{n}^{T}\right] \longrightarrow \Lambda^{*}$ by $\mathbb{N} G\left(\boldsymbol{n}^{T}\right)(u, \boldsymbol{\xi})=\boldsymbol{n}^{T}(u)+\boldsymbol{\xi}$, which we call the nullcone Gauss image of $N_{1}(M)\left[\boldsymbol{n}^{T}\right]$. This map leads us to the notions of curvatures. Let $T_{(p, \xi)} N_{1}(M)\left[\boldsymbol{n}^{T}\right]$ be the tangent space of $N_{1}(M)\left[\boldsymbol{n}^{T}\right]$ at $(p, \boldsymbol{\xi})$. Under the canonical identification $\left(\mathbb{N G}\left(\boldsymbol{n}^{T}\right)^{*} T \mathbb{R}_{2}^{n+2}\right)_{(p, \boldsymbol{\xi})}=T_{\left(\boldsymbol{n}^{T}(p)+\boldsymbol{\xi}\right)} \mathbb{R}_{2}^{n+2} \equiv T_{p} \mathbb{R}_{2}^{n+2}$, we have

$$
T_{(p, \xi)} N_{1}(M)\left[\boldsymbol{n}^{T}\right]=T_{p} M \oplus T_{\xi} S^{k-2} \subset T_{p} M \oplus N_{p}(M)=T_{p} \mathbb{R}_{2}^{n+2},
$$

where $T_{\xi} S^{k-2} \subset T_{\xi} N_{p}(M) \equiv N_{p}(M)$ and $p=X(u)$. Let

$$
\Pi^{t}: \mathbb{N} G\left(\boldsymbol{n}^{T}\right)^{*} T \mathbb{R}_{2}^{n+2}=T N_{1}(M)\left[\boldsymbol{n}^{T}\right] \oplus \mathbb{R}^{k+1} \longrightarrow T N_{1}(M)\left[\boldsymbol{n}^{T}\right]
$$

be the canonical projection. Then, we have a linear transformation

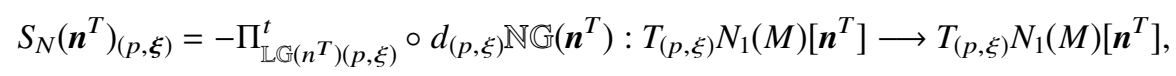

which is called the nullcone shape operator of $N_{1}(M)\left[\boldsymbol{n}^{T}\right]$ at $(p, \boldsymbol{\xi})$. Let $\kappa_{N}\left(\boldsymbol{n}^{T}\right)_{i}(p, \boldsymbol{\xi})$ be the eigenvalues of $S_{N}\left(\boldsymbol{n}^{T}\right)_{(p, \boldsymbol{\xi})},(i=1, \ldots, n-1)$. Here, we denote $\kappa_{N}\left(\boldsymbol{n}^{T}\right)_{i}(p, \boldsymbol{\xi}),(i=1, \ldots, s)$ as the eigenvalues belonging to the eigenvectors on $T_{p} M$ and $\kappa_{N}\left(\boldsymbol{n}^{T}\right)_{i}(p, \boldsymbol{\xi}),(i=s+1, \ldots, n-1)$ as the eigenvalues belonging to the eigenvectors on the tangent space of the fiber of $N_{1}(M)\left[\boldsymbol{n}^{T}\right]$. Then, we have the following proposition.

Proposition 3.2. We choose a (local) pseudo-orthonormal frame $\left\{\boldsymbol{X}, \boldsymbol{n}^{T}, \boldsymbol{n}_{1}^{S}, \ldots, \boldsymbol{n}_{k-1}^{S}\right\}$ of $N(M)$ with $\boldsymbol{n}_{k-1}^{S}=\boldsymbol{n}^{S}$. For $p_{0}=\boldsymbol{X}\left(u_{0}\right)$ and $\boldsymbol{\xi}_{0}=\boldsymbol{n}^{S}\left(u_{0}\right)$, we have $\kappa_{N}\left(\boldsymbol{n}^{T}\right)_{i}\left(p_{0}, \boldsymbol{\xi}_{0}\right)=\kappa_{i}\left(\boldsymbol{n}^{T}, \boldsymbol{n}^{S}\right)\left(u_{0}\right),(i=1, \ldots$, $s)$ and $\kappa_{N}\left(\boldsymbol{n}^{T}\right)_{i}\left(p_{0}, \boldsymbol{\xi}_{0}\right)=-1,(i=s+1, \ldots, n-1)$.

Proof. Since $\left\{\boldsymbol{X}, \boldsymbol{n}^{T}, \boldsymbol{n}_{1}^{S}, \ldots, \boldsymbol{n}_{k-1}^{S}\right\}$ is a pseudo-orthonormal frame of $N(M)$, we have $\left\langle\boldsymbol{X}\left(u_{0}\right), \boldsymbol{\xi}_{0}\right\rangle$ $=\left\langle\boldsymbol{n}^{T}\left(u_{0}\right), \boldsymbol{\xi}_{0}\right\rangle=\left\langle\boldsymbol{n}_{i}^{S}\left(u_{0}\right), \boldsymbol{\xi}_{0}\right\rangle=0$. Therefore, we have $T_{\boldsymbol{\xi}} S^{k-2}=\left\langle\boldsymbol{n}_{1}^{S}\left(u_{0}\right), \ldots, \boldsymbol{n}_{k-2}^{S}\left(u_{0}\right)\right\rangle$. By this orthonormal basis of $T_{\xi_{0}} S^{k-2}$, the canonical Riemannian metric $G_{i j}\left(p_{0}, \xi_{0}\right)$ is represented by

$$
\left(G_{i j}\left(p_{0}, \boldsymbol{\xi}\right)\right)=\left(\begin{array}{cc}
g_{i j}\left(p_{0}\right) & 0 \\
0 & I_{k-2}
\end{array}\right),
$$

where $g_{i j}\left(p_{0}\right)=\left\langle\boldsymbol{X}_{u_{i}}\left(u_{0}\right), \boldsymbol{X}_{u_{j}}\left(u_{0}\right)\right\rangle$.

On the other hand, by Proposition 3.1, we have

$$
-\sum_{j=1}^{s} h_{i}^{j}\left(\boldsymbol{n}^{T}, \boldsymbol{n}^{S}\right)\left(u_{0}\right) \boldsymbol{X}_{u_{j}}=\mathbb{N} G\left(\boldsymbol{n}^{T}, \boldsymbol{n}^{S}\right)_{u_{i}}\left(u_{0}\right)=d_{p_{0}} \mathbb{N} G\left(\boldsymbol{n}^{T}, \boldsymbol{n}^{S}\right)\left(\frac{\partial}{\partial u_{i}}\right),
$$

so that we have

$$
S_{N}\left(\boldsymbol{n}^{T}\right)_{\left(p_{0}, \xi_{0}\right)}\left(\frac{\partial}{\partial u_{i}}\right)=\sum_{j=1}^{s} h_{i}^{j}\left(\boldsymbol{n}^{T}, \boldsymbol{n}^{S}\right)\left(u_{0}\right) \boldsymbol{X}_{u_{j}}
$$

Therefore, the representation matrix of $S_{N}\left(\boldsymbol{n}^{T}\right)_{\left(p_{0}, \xi_{0}\right)}$ with respect to the basis

$$
\left\{\boldsymbol{X}_{u_{1}}\left(u_{0}\right), \ldots, \boldsymbol{X}_{u_{s}}\left(u_{0}\right), \boldsymbol{n}_{1}^{S}\left(u_{0}\right), \ldots, \boldsymbol{n}_{k-2}^{S}\left(u_{0}\right)\right\}
$$

of $T_{\left(p_{0}, \xi_{0}\right)}\left(N_{1}(M)\left[\boldsymbol{n}^{T}\right]\right)$ is of the form 


$$
\left(\begin{array}{cc}
h_{i}^{j}\left(\boldsymbol{n}^{T}, \boldsymbol{n}^{S}\right)\left(u_{0}\right) & * \\
0 & -I_{k-2}
\end{array}\right) .
$$

Thus, the eigenvalues of this matrix are $\lambda_{i}=\kappa_{i}\left(\boldsymbol{n}^{T}, \boldsymbol{n}^{S}\right)\left(u_{0}\right),(i=1, \ldots, s)$ and $\lambda_{i}=-1,(i=s+$ $1, \ldots, n-1)$. This completes the proof.

We call $\kappa_{N}\left(\boldsymbol{n}^{T}\right)_{i}(p, \xi),(i=1, \ldots, s)$ the nullcone principal curvatures of $M$ with respect to $\left(\boldsymbol{n}^{T}, \boldsymbol{\xi}\right)$ at $p \in M$. The nullcone Lipschitz-Killing curvature of $N_{1}(M)\left[\boldsymbol{n}^{T}\right]$ at $(p, \boldsymbol{\xi})$ is defined to be $K_{N}\left(\boldsymbol{n}^{T}\right)(p, \boldsymbol{\xi})=\operatorname{det} S_{N}\left(\boldsymbol{n}^{T}\right)_{(p, \xi)}$.

\section{LIGHTLIKE HYPERSURFACES IN ANTI-DE SITTER SPACE}

We define a hypersurface

$$
\mathbb{L} \mathbb{H}_{M}\left(\boldsymbol{n}^{T}\right): N_{1}(M)\left[\boldsymbol{n}^{T}\right] \times \mathbb{R} \longrightarrow A d S^{n+1}
$$

by

$$
\mathbb{L H}_{M}\left(\boldsymbol{n}^{T}\right)((p, \boldsymbol{\xi}), \mu)=\boldsymbol{X}(u)+\mu\left(\boldsymbol{n}^{T}+\boldsymbol{\xi}\right)(u)=\boldsymbol{X}(u)+\mu \mathbb{N} G\left(\boldsymbol{n}^{T}\right)(u, \boldsymbol{\xi}),
$$

where $p=\boldsymbol{X}(u)$, which is called the lightlike hypersurface along $M$ relative to $\boldsymbol{n}^{T}$. In general, a hypersurface $H \subset A d S^{n+1}$ is called a lightlike hypersurface if it is tangent to the lightcone at any regular point. We remark that $\mathbb{N H}_{M}\left(\boldsymbol{n}^{T}\right)\left(N_{1}(M)\left[\boldsymbol{n}^{T}\right] \times \mathbb{R}\right)$ is a lightlike hypersurface.

We introduce the notion of height functions on a spacelike submanifold, which is useful for the study of singularities of lightlike hypersurfaces. We define a family of functions

$$
H: M \times A d S^{n+1} \longrightarrow \mathbb{R}
$$

on a spacelike submanifold $M=\boldsymbol{X}(U)$ by $H(p, \lambda)=H(u, \lambda)=\langle\boldsymbol{X}(u), \lambda\rangle+1$, where $p=\boldsymbol{X}(u)$. We call $H$ the anti-de Sitter height function (briefly, AdS-height function) on the spacelike submanifold $M$. For any fixed $\lambda_{0} \in A d S^{n+1}$, we write $h_{\lambda_{0}}(p)=H\left(p, \lambda_{0}\right)$ and have the following proposition.

Proposition 4.1. Let $M$ be a spacelike submanifold and $H: M \times\left(A d S^{n+1} \backslash M\right) \rightarrow \mathbb{R}$ the AdSheight function on $M$. Suppose that $p_{0}=X\left(u_{0}\right) \neq \lambda_{0}$. Then, we have the following:

(1) $\quad h_{\lambda_{0}}\left(p_{0}\right)=\partial h_{\lambda_{0}} / \partial u_{i}\left(p_{0}\right)=0,(i=1, \ldots, s)$ if and only if there exist $\xi_{0} \in N_{1}(M)_{p_{0}}\left[\boldsymbol{n}^{T}\right]$ and $\mu_{0} \in \mathbb{R} \backslash\{0\}$ such that

$$
\lambda_{0}=\boldsymbol{X}\left(u_{0}\right)+\mu_{0} \mathbb{N G}\left(\boldsymbol{n}^{T}\right)\left(u_{0}, \boldsymbol{\xi}_{0}\right)=\mathbb{L} \mathbb{H} \mathbb{M}_{M}\left(\boldsymbol{n}^{T}\right)\left(\left(p_{0}, \boldsymbol{\xi}_{0}\right), \mu_{0}\right) .
$$

(2) $\quad h_{\lambda_{0}}\left(p_{0}\right)=\partial h_{\lambda_{0}} / \partial u_{i}\left(p_{0}\right)=\operatorname{det} \mathcal{H}\left(h_{\lambda_{0}}\right)\left(p_{0}\right)=0(i=1, \ldots, s)$ if and only if there exist $\xi_{0} \in$ $N_{1}(M)_{p_{0}}\left[\boldsymbol{n}^{T}\right]$ and $\mu_{0} \in \mathbb{R} \backslash\{0\}$ such that

$$
\lambda_{0}=\mathbb{L H}_{M}\left(\boldsymbol{n}^{T}\right)\left(\left(p_{0}, \boldsymbol{\xi}_{0}\right), \mu_{0}\right)
$$

and $1 / \mu$ is one of the non-zero nullcone principal curvatures $\kappa_{N}\left(\boldsymbol{n}^{T}\right)_{i}\left(p_{0}, \boldsymbol{\xi}_{0}\right),(i=1, \ldots, s)$.

Here, $\mathcal{H}\left(h_{\lambda_{0}}\right)\left(p_{0}\right)$ is the Hessian matrix of $h_{\lambda_{0}}$ at $p_{0}$.

(3) With condition (2), rank $\mathcal{H}\left(h_{\lambda_{0}}\right)\left(p_{0}\right)=0$ if and only if $p_{0}=\boldsymbol{X}\left(u_{0}\right)$ is a non-flat $\left(\boldsymbol{n}^{T}\left(u_{0}\right), \boldsymbol{\xi}_{0}\right)$ umbilical point.

Proof. (1) For $p=\boldsymbol{X}(u)$, the condition $h_{\lambda_{0}}(p)=\left\langle\boldsymbol{X}(u), \lambda_{0}\right\rangle+1=0$ means that

$$
\left\langle\boldsymbol{X}(u)-\lambda_{0}, \boldsymbol{X}(u)-\lambda_{0}\right\rangle=\langle\boldsymbol{X}(u), \boldsymbol{X}(u)\rangle-2\left\langle\boldsymbol{X}(u), \lambda_{0}\right\rangle+\left\langle\lambda_{0}, \lambda_{0}\right\rangle=-2\left(1+\left\langle\boldsymbol{X}(u), \lambda_{0}\right\rangle\right)=0,
$$

so that $\boldsymbol{X}(u)-\lambda_{0} \in \Lambda^{*}$. Since $\partial h_{\lambda_{0}} / \partial u_{i}(p)=\left\langle\boldsymbol{X}_{u_{i}}(u), \lambda_{0}\right\rangle$ and $\left\langle\boldsymbol{X}_{u_{i}}, \boldsymbol{X}\right\rangle=0$, we have $\left\langle\boldsymbol{X}_{u_{i}}(u), \lambda_{0}\right\rangle$ $=-\left\langle\boldsymbol{X}_{u_{i}}(u)-\lambda_{0}\right\rangle$. Therefore, $\partial h_{\lambda_{0}} / \partial u_{i}(p)=0$ if and only if $\boldsymbol{X}(u)-\lambda_{0} \in N_{p} M$. On the other hand, the condition $h_{\lambda_{0}}(p)=\left\langle\boldsymbol{X}(u), \lambda_{0}\right\rangle+1=0$ implies that $\left\langle\boldsymbol{X}(u), \boldsymbol{X}(u)-\boldsymbol{\lambda}_{0}\right\rangle=0$. This means that $\boldsymbol{X}(u)-\lambda_{0} \in T_{p} A d S^{n+1}$. Hence, $h_{\lambda_{0}}\left(p_{0}\right)=\partial h_{\lambda_{0}} / \partial u_{i}\left(p_{0}\right)=0(i=1, \ldots, s)$ if and only if $\boldsymbol{X}\left(u_{0}\right)-\lambda_{0} \in N_{p_{0}} M \cap \Lambda^{*} \cap T_{p_{0}} A d S^{n+1}$. Then, we denote that $\boldsymbol{v}=\boldsymbol{X}\left(u_{0}\right)-\lambda_{0} \in N_{p_{0}} M \cap \Lambda^{*} \cap$ $T_{p_{0}} A d S^{n+1}$. If $\left\langle\boldsymbol{n}^{T}\left(u_{0}\right), \boldsymbol{v}\right\rangle=0$, then $\boldsymbol{n}^{T}\left(u_{0}\right)$ belongs to a lightlike hyperplane in the Lorentz 
space $T_{p_{0}} A d S^{n+1}$, so that $\boldsymbol{n}^{T}\left(u_{0}\right)$ is lightlike or spacelike. This is in contradiction to the fact that $\boldsymbol{n}^{T}\left(u_{0}\right)$ is a timelike unit vector. Thus, $\left\langle\boldsymbol{n}^{T}\left(u_{0}\right), \boldsymbol{v}\right\rangle \neq 0$. We set

$$
\xi_{0}=\frac{-1}{\left\langle\boldsymbol{n}^{T}\left(u_{0}\right), \boldsymbol{v}\right\rangle} \boldsymbol{v}-\boldsymbol{n}^{T}\left(u_{0}\right)
$$

Then, we have

$$
\begin{aligned}
\left\langle\boldsymbol{\xi}_{0}, \boldsymbol{\xi}_{0}\right\rangle & =-2 \frac{-1}{\left\langle\boldsymbol{n}^{T}\left(u_{0}\right), \boldsymbol{v}\right\rangle}\left\langle\boldsymbol{n}^{T}\left(u_{0}\right), \boldsymbol{v}\right\rangle-1=1, \\
\left\langle\boldsymbol{\xi}_{0}, \boldsymbol{n}^{T}\left(u_{0}\right)\right\rangle & =\frac{-1}{\left\langle\boldsymbol{n}^{T}\left(u_{0}\right), \boldsymbol{v}\right\rangle}\left\langle\boldsymbol{n}^{T}\left(u_{0}\right), \boldsymbol{v}\right\rangle+1=0 .
\end{aligned}
$$

This means that $\xi_{0} \in N_{1}(M)_{p_{0}}(M)\left[\boldsymbol{n}^{T}\right]$. Since $-\boldsymbol{v}=\left\langle\boldsymbol{n}^{T}\left(u_{0}\right), \boldsymbol{v}\right\rangle\left(\boldsymbol{n}^{T}\left(u_{0}\right)+\boldsymbol{\xi}_{0}\right)$, we have $\lambda_{0}=$ $\boldsymbol{X}\left(u_{0}\right)+\mu_{0} \mathbb{N} G\left(\boldsymbol{n}^{T}\right)\left(p_{0}, \boldsymbol{\xi}_{0}\right)$, where $p_{0}=\boldsymbol{X}\left(u_{0}\right)$ and $\mu_{0}=\left\langle\boldsymbol{n}^{T}\left(u_{0}\right), \boldsymbol{v}\right\rangle$. For the converse assertion, suppose that $\lambda_{0}=\boldsymbol{X}\left(u_{0}\right)+\mu_{0} \mathbb{N} G\left(n^{T}\right)\left(p_{0}, \xi_{0}\right)$. Then $\lambda_{0}-X\left(u_{0}\right) \in N_{p_{0}}(M) \cap \Lambda^{*}$ and $\left\langle\lambda_{0}-\right.$ $\left.\boldsymbol{X}\left(u_{0}\right), \boldsymbol{X}\left(u_{0}\right)\right\rangle=\left\langle\mu_{0} \mathbb{N} G\left(\boldsymbol{n}^{T}\right)\left(p_{0}, \boldsymbol{\xi}_{0}\right), \boldsymbol{X}\left(u_{0}\right)\right\rangle=0$. Thus, we have $\lambda_{0}-\boldsymbol{X}\left(u_{0}\right) \in N_{p_{0}}(M) \cap \Lambda^{*} \cap$ $T_{p_{0}} A d S^{n+1}$. By the previous arguments, these conditions are equivalent to the condition that $h_{\lambda_{0}}\left(p_{0}\right)=\partial h_{\lambda_{0}} / \partial u_{i}\left(p_{0}\right)=0(i=1, \ldots, s)$.

(2) By a straightforward calculation, we have

$$
\frac{\partial^{2} h_{\lambda_{0}}}{\partial u_{i} \partial u_{j}}(u)=\left\langle\boldsymbol{X}_{u_{i} u_{j}}, \lambda_{0}\right\rangle .
$$

Under the conditions $\lambda_{0}=\boldsymbol{X}\left(u_{0}\right)+\mu_{0}\left(\boldsymbol{n}^{T}\left(u_{0}\right)+\xi_{0}\right)$, we have

$$
\frac{\partial^{2} h_{\boldsymbol{\lambda}_{0}}}{\partial u_{i} \partial u_{j}}\left(u_{0}\right)=\left\langle\boldsymbol{X}_{u_{i} u_{j}}\left(u_{0}\right), \boldsymbol{X}\left(u_{0}\right)\right\rangle+\mu_{0}\left\langle\boldsymbol{X}_{u_{i} u_{j}}\left(u_{0}\right),\left(\boldsymbol{n}^{T}\left(u_{0}\right)+\boldsymbol{\xi}_{0}\right)\right\rangle .
$$

Since $\left\langle\boldsymbol{X}_{u_{i}}, \boldsymbol{X}\right\rangle=0$, we have $\left\langle\boldsymbol{X}_{u_{i} u_{j}}, \boldsymbol{X}\right\rangle=-\left\langle\boldsymbol{X}_{u_{i}}, \boldsymbol{X}_{u_{j}}\right\rangle$. Therefore, we have

$$
\left(\frac{\partial^{2} h_{\lambda_{0}}}{\partial u_{i} \partial u_{\ell}}\left(u_{0}\right)\right)\left(g^{j \ell}\left(u_{0}\right)\right)=\left(\mu_{0} h_{i}^{j}\left(\boldsymbol{n}^{T}, \boldsymbol{n}^{S}\right)\left(u_{0}\right)-\delta_{i}^{j}\right),
$$

where $\boldsymbol{n}^{S}$ is the local section of $N_{1}(M)\left[\boldsymbol{n}^{T}\right]$, with $\boldsymbol{n}^{S}\left(u_{0}\right)=\boldsymbol{\xi}_{0}$. It follows that $\operatorname{det} \mathcal{H}(g)\left(p_{0}\right)=0$ if and only if $1 / \mu_{0}$ is an eigenvalue of $\left(h_{j}^{i}\left(\boldsymbol{n}^{T}, \boldsymbol{n}^{S}\right)\left(u_{0}\right)\right)$, which is equal to one of the nullcone principal curvatures $\kappa_{i}\left(\boldsymbol{n}^{T}, \boldsymbol{n}^{S}\right)\left(u_{0}\right)=\kappa_{N}\left(\boldsymbol{n}^{T}\right)_{i}\left(p_{0}, \boldsymbol{\xi}_{0}\right),(i=1, \ldots, s)$. By the above calculation, rank $\mathcal{H}\left(h_{\lambda_{0}}\right)\left(p_{0}\right)=0$ if and only if

$$
\left(h_{j}^{i}\left(\boldsymbol{n}^{T}, \boldsymbol{n}^{S}\right)\left(u_{0}\right)\right)=\frac{1}{\mu_{0}}\left(\delta_{i}^{j}\right),
$$

where $1 / \mu_{0}=\kappa_{N}\left(\boldsymbol{n}^{T}\right)_{i}\left(p_{0}, \boldsymbol{\xi}_{0}\right),(i=1, \ldots, s)$. This means that $p_{0}=\boldsymbol{X}\left(u_{0}\right)$ is an $\left(\boldsymbol{n}^{T}\left(u_{0}\right), \boldsymbol{\xi}_{0}\right)$ umbilical point.

In order to understand the geometric meaning of the assertions of Proposition 4.1, we briefly review the theory of Legendrian singularities. For detailed expressions, see Refs. 1 and 32. Let $\pi: P T^{*}\left(\mathbb{R}^{n+1}\right) \longrightarrow \mathbb{R}^{n+1}$ be the projective cotangent bundle with its canonical contact structure. We next review the geometric properties of this bundle. Consider the tangent bundle $\tau: T P T^{*}\left(\mathbb{R}^{n+}\right) \rightarrow$ $P T^{*}\left(\mathbb{R}^{n+1}\right)$ and the differential map $d \pi: T P T^{*}\left(\mathbb{R}^{n+1}\right) \rightarrow T \mathbb{R}^{n+1}$ of $\pi$. For any $X \in T P T^{*}\left(\mathbb{R}^{n+1}\right)$, there exists an element $\alpha \in T^{*}\left(\mathbb{R}_{1}^{n+1}\right)$ such that $\tau(X)=[\alpha]$. For an element $V \in T_{x}\left(\mathbb{R}^{n+1}\right)$, the property $\alpha(V)=0$ does not depend on the choice of representative of the class $[\alpha]$. Thus, we can define the canonical contact structure on $P T^{*}\left(\mathbb{R}^{n+1}\right)$ by

$$
K=\left\{X \in T P T^{*}\left(\mathbb{R}^{n+1}\right) \mid \tau(X)(d \pi(X))=0\right\} .
$$

We have a trivialization $P T^{*}\left(\mathbb{R}^{n+1}\right) \cong \mathbb{R}^{n+1} \times P^{n}(\mathbb{R})^{*}$, and call

$$
\left(\left(v_{0}, v_{1}, \ldots, v_{n}\right),\left[\xi_{0}: \xi_{1}: \cdots: \xi_{n}\right]\right)
$$

the homogeneous coordinates of $P T^{*}\left(\mathbb{R}^{n+1}\right)$, where $\left[\xi_{0}: \xi_{1}: \cdots: \xi_{n}\right]$ are the homogeneous coordinates of the dual projective space $P^{n}(\mathbb{R})^{*}$. It is easy to show that $X \in K_{(x,[\xi])}$ if and only if 
$\sum_{i=0}^{n} \mu_{i} \xi_{i}=0$, where $d \tilde{\pi}(X)=\sum_{i=0}^{n} \mu_{i} \partial / \partial v_{i}$. An immersion $i: L \rightarrow P T^{*}\left(\mathbb{R}^{n+1}\right)$ is said to be $a$ Legendrian immersion if $\operatorname{dim} L=n$ and $d i_{q}\left(T_{q} L\right) \subset K_{i(q)}$ for any $q \in L$. The map $\pi \circ i$ is also called the Legendrian map and the set $W(i)=$ image $\pi \circ i$, the wave front set of $i$. Moreover, $i$ (or, the image of $i)$ is called the Legendrian lift of $W(i)$.

Let $F:\left(\mathbb{R}^{k} \times \mathbb{R}^{n+1}, \mathbf{0}\right) \longrightarrow(\mathbb{R}, \mathbf{0})$ be a function germ. We say that $F$ is a Morse family of hypersurfaces if the map germ

$$
\Delta^{*} F=\left(F, \frac{\partial F}{\partial q_{1}}, \ldots, \frac{\partial F}{\partial q_{k}}\right):\left(\mathbb{R}^{k} \times \mathbb{R}^{n+1}, \mathbf{0}\right) \longrightarrow\left(\mathbb{R} \times \mathbb{R}^{k}, \mathbf{0}\right)
$$

is submersive, where $(q, x)=\left(q_{1}, \ldots, q_{k}, x_{0}, \ldots, x_{n}\right) \in\left(\mathbb{R}^{k} \times \mathbb{R}^{n+1}, \mathbf{0}\right)$. In this case, we have a smooth $n$-dimensional submanifold

$$
\Sigma_{*}(F)=\left\{(q, x) \in\left(\mathbb{R}^{k} \times \mathbb{R}^{n+1}, \mathbf{0}\right) \mid F(q, x)=\frac{\partial F}{\partial q_{1}}(q, x)=\cdots=\frac{\partial F}{\partial q_{k}}(q, x)=0\right\}
$$

and the map germ $\mathscr{L}_{F}:\left(\Sigma_{*}(F), \boldsymbol{0}\right) \longrightarrow P T^{*} \mathbb{R}^{n+1}$ defined by

$$
\mathscr{L}_{F}(q, x)=\left(x,\left[\frac{\partial F}{\partial x_{0}}(q, x): \cdots: \frac{\partial F}{\partial x_{n}}(q, x)\right]\right)
$$

is a Legendrian immersion. We call $F$ a generating family of $\mathscr{L}_{F}\left(\Sigma_{*}(F)\right)$, and the wave front set is given by $W\left(\mathscr{L}_{F}\right)=\pi_{n}\left(\Sigma_{*}(F)\right)$, where $\pi_{n}: \mathbb{R}^{k} \times \mathbb{R}^{n} \longrightarrow \mathbb{R}^{n}$ is the canonical projection. In the theory of unfoldings of function germs, the wave front set $W\left(\mathscr{L}_{F}\right)$ is called a discriminant set of $F$, which we also denote $\mathcal{D}_{F}$. Therefore, Proposition 4.1 asserts that the discriminant set of the AdS-height function $H$ is given by

$$
\mathcal{D}_{H}=\left\{\lambda \in A d S^{n+1} \mid \lambda=\boldsymbol{X}(u)+\mu\left(\boldsymbol{n}^{T} \pm \boldsymbol{\xi}\right)(u), p=\boldsymbol{X}(u) \in M, \boldsymbol{\xi} \in N_{1}(M)_{p}\left[\boldsymbol{n}^{T}\right], \mu \in \mathbb{R}\right\},
$$

which is the image of the lightlike hypersurface along $M$ relative to $\boldsymbol{n}^{T}$.

By assertion (2) of Proposition 4.1, a singular point of the lightlike hypersurface is a point $\lambda_{0}=\boldsymbol{X}\left(u_{0}\right)+\mu_{0}\left(\boldsymbol{n}^{T}+\boldsymbol{\xi}_{0}\right)\left(u_{0}\right)$ for $p_{0}=\boldsymbol{X}\left(u_{0}\right)$ and $\left.\mu_{0}=1 / \kappa_{N}\left(\boldsymbol{n}^{T}\right)_{i}\left(p_{0}, \boldsymbol{\xi}_{0}\right), i=1, \ldots, s\right)$. Then, we have the following corollary.

Corollary 4.2. The critical value of $\mathbb{L} \mathbb{H}_{M}\left(\boldsymbol{n}^{T}\right)$ is the point

$$
\lambda=\boldsymbol{X}(u)+\frac{1}{\kappa_{N}\left(\boldsymbol{n}^{T}\right)_{i}(p, \boldsymbol{\xi})} \mathbb{L} G\left(\boldsymbol{n}^{T}\right)(u, \boldsymbol{\xi}),
$$

where $p=\boldsymbol{X}(u)$ and $\kappa_{N}\left(\boldsymbol{n}^{T}\right)_{i}(p, \boldsymbol{\xi}) \neq 0$.

For a non-zero nullcone principal curvature $\kappa_{N}\left(\boldsymbol{n}^{T}\right)_{i}\left(p_{0}, \boldsymbol{\xi}_{0}\right) \neq 0$, we have an open subset $O_{i} \subset$ $N_{1}(M)\left[\boldsymbol{n}^{T}\right]$ such that $\kappa_{N}\left(\boldsymbol{n}^{T}\right)(p, \boldsymbol{\xi}) \neq 0$. Therefore, we have a non-zero nullcone principal curvature function $\kappa_{N}\left(\boldsymbol{n}^{T}\right): O_{i} \longrightarrow \mathbb{R}$. We define a mapping $\mathbb{L F}_{\kappa_{N}\left(\boldsymbol{n}^{T}\right)_{i}}: O_{i} \longrightarrow A d S^{n+1}$ by

$$
\mathbb{L F}_{\kappa_{N}\left(\boldsymbol{n}^{T}\right)_{i}}(p, \boldsymbol{\xi})=\boldsymbol{X}(u)+\frac{1}{\kappa_{N}\left(\boldsymbol{n}^{T}\right)_{i}(p, \boldsymbol{\xi})} \mathbb{N} G\left(\boldsymbol{n}^{T}\right)(u, \boldsymbol{\xi}),
$$

where $p=\boldsymbol{X}(u)$. We also define

$$
\mathbb{L} \mathbb{F}_{M}\left(\boldsymbol{n}^{T}\right)=\bigcup\left\{\mathbb{L F}_{\kappa_{N}\left(\boldsymbol{n}^{T}\right)_{i}}(p, \boldsymbol{\xi}) \mid(p, \boldsymbol{\xi}) \in N_{1}(M)\left[\boldsymbol{n}^{T}\right] \text { s.t. } \kappa_{N}\left(\boldsymbol{n}^{T}\right)_{i}(p, \boldsymbol{\xi}) \neq 0, i=1, \ldots, s\right\} .
$$

We call $\mathbb{L} \mathbb{F}_{M}\left(\boldsymbol{n}^{T}\right)$ the lightlike focal set of $M=X(U)$ relative to $\boldsymbol{n}^{T}$. By definition, the lightlike focal set of $M=\boldsymbol{X}(U)$ relative to $\boldsymbol{n}^{T}$ is the critical values set of the lightlike hypersurface $\mathbb{L H}_{M}\left(\boldsymbol{n}^{T}\right)\left(N_{1}(M)\left[\boldsymbol{n}^{T}\right] \times \mathbb{R}\right)$ along $M$ relative to $\boldsymbol{n}^{T}$.

We can show that the image of the lightlike hypersurface along $M$ is independent of the choice of the future directed timelike normal vector field $\boldsymbol{n}^{T}$ as a corollary of Proposition 4.1. have

Corollary 4.3. Let $\boldsymbol{n}^{T}$ and $\overline{\boldsymbol{n}}^{T}$ be future directed timelike unit normal fields along M. Then, we

$$
\mathbb{L H}_{M}\left(\boldsymbol{n}^{T}\right)\left(N_{1}(M)\left[\boldsymbol{n}^{T}\right] \times \mathbb{R}\right)=\mathbb{L} \mathbb{H}_{M}\left(\overline{\boldsymbol{n}}^{T}\right)\left(N_{1}(M)\left[\overline{\boldsymbol{n}}^{T}\right] \times \mathbb{R}\right) \text { and } \mathbb{L} \mathbb{F}_{M}\left(\boldsymbol{n}^{T}\right)=\mathbb{L} \mathbb{F}_{M}\left(\overline{\boldsymbol{n}}^{T}\right) .
$$


Proof. By Proposition 4.1, the images of the lightlike hypersurface along $M$ relative to $\boldsymbol{n}^{T}$ and $\bar{n}^{T}$ are the discriminant sets of the AdS-height function $H$ on $M$. Moreover, the focal set is the critical value set of the lightlike hypersurface along $M$ relative to $\boldsymbol{n}^{T}$. Since $H$ is independent of the choice of $\boldsymbol{n}^{T}$, we have the assertion.

We have the following proposition.

Proposition 4.4. Let $H$ be the AdS-height function on M. For any point $(u, \lambda) \in \Delta^{*} H^{-1}(0)$, the germ of $H$ at $(u, \lambda)$ is a Morse family of hypersurfaces.

Proof. We denote that

$$
\boldsymbol{X}(u)=\left(X_{-1}(u), X_{0}(u), X_{1}(u), \ldots, X_{n}(u)\right) \text { and } \lambda=\left(\lambda_{-1}, \lambda_{0}, \lambda_{1}, \ldots, \lambda_{n}\right) .
$$

We define an open subset $U_{-1}^{+}=\left\{\lambda \in A d S^{n+1} \mid \lambda_{-1}>0\right\}$. For any $\lambda \in U_{-1}^{+}$, we have

$$
\lambda_{-1}=\sqrt{1-\lambda_{0}^{2}+\lambda_{1}^{2}+\cdots \lambda_{n}^{2}}
$$

Thus, we have a local coordinate of $A d S^{n+1}$ given by $\left(\lambda_{0}, \lambda_{1}, \ldots, \lambda_{n}\right)$ on $U_{-1}^{+}$. By definition, we have

$$
H(u, \lambda)=-X_{-1}(u) \sqrt{1-\lambda_{0}^{2}+\sum_{i=1}^{n} \lambda_{i}^{2}}-X_{0}(u) \lambda_{0}+X_{1}(u) \lambda_{1}+\cdots+X_{n}(u) \lambda_{n} .
$$

We now prove that the mapping

$$
\Delta^{*} H=\left(H, \frac{\partial H}{\partial u_{1}}, \ldots, \frac{\partial H}{\partial u_{s}}\right)
$$

is non-singular at $(u, \lambda) \in \Delta^{*} H^{-1}(0)$. Indeed, the Jacobian matrix of $\Delta^{*} H$ is given by

$$
\left(\begin{array}{cccc}
X_{-1} \frac{\lambda_{0}}{\lambda_{-1}}-X_{0} & -X_{-1} \frac{\lambda_{1}}{\lambda_{-1}}+X_{1} & \cdots & -X_{-1} \frac{\lambda_{n}}{\lambda_{-1}}-X_{n} \\
X_{-1 u_{1}} \frac{\lambda_{0}}{\lambda_{-1}}-X_{0 u_{1}} & -X_{-1 u_{1}} \frac{\lambda_{1}}{\lambda_{-1}}+X_{1 u_{1}} & \cdots & -X_{-1 u_{1}} \frac{\lambda_{n}}{\lambda_{-1}}-X_{n u_{1}} \\
\vdots & \vdots & \ddots & \vdots \\
X_{-1 u_{s}} \frac{\lambda_{0}}{\lambda_{-1}}-X_{0 u_{s}} & -X_{-1 u_{s}} \frac{\lambda_{1}}{\lambda_{-1}}+X_{1 u_{s}} & \cdots & -X_{-1 u_{s}} \frac{\lambda_{n}}{\lambda_{-1}}-X_{n u_{s}}
\end{array}\right)
$$

where

$$
\mathbf{A}=\left(\begin{array}{ccc}
\left\langle\boldsymbol{X}_{u_{1}}, \lambda\right\rangle & \cdots & \left\langle\boldsymbol{X}_{u_{s}}, \lambda\right\rangle \\
\left\langle\boldsymbol{X}_{u_{1} u_{1}}, \lambda\right\rangle & \cdots & \left\langle\boldsymbol{X}_{u_{1} u_{s}}, \lambda\right\rangle \\
\vdots & \ddots & \vdots \\
\left\langle\boldsymbol{X}_{u_{s} u_{1}}, \lambda\right\rangle & \cdots & \left\langle\boldsymbol{X}_{u_{s} u_{s}}, \lambda\right\rangle
\end{array}\right)
$$

We now show that the rank of

$$
\mathbf{B}=\left(\begin{array}{cccc}
X_{-1} \frac{\lambda_{0}}{\lambda_{-1}}-X_{0} & -X_{-1} \frac{\lambda_{1}}{\lambda_{-1}}+X_{1} & \cdots & -X_{-1} \frac{\lambda_{n}}{\lambda_{-1}}-X_{n} \\
X_{-1 u_{1}} \frac{\lambda_{0}}{\lambda_{-1}}-X_{0 u_{1}} & -X_{-1 u_{1}} \frac{\lambda_{1}}{\lambda_{-1}}+X_{1 u_{1}} & \cdots & -X_{-1 u_{1}} \frac{\lambda_{n}}{\lambda_{-1}}-X_{n u_{1}} \\
\vdots & \vdots & \ddots & \vdots \\
X_{-1 u_{s}} \frac{\lambda_{0}}{\lambda_{-1}}-X_{0 u_{s}} & -X_{-1 u_{s}} \frac{\lambda_{1}}{\lambda_{-1}}+X_{1 u_{s}} & \cdots & -X_{-1 u_{s}} \frac{\lambda_{n}}{\lambda_{-1}}-X_{n u_{s}}
\end{array}\right)
$$

is $s+1$ at $(u, \lambda) \in \Sigma_{*}(H)$. Since $(u, \lambda) \in \Sigma_{*}(H)$, we have

$$
\lambda=\boldsymbol{X}(u)+\mu\left(\boldsymbol{n}^{T}(u)+\sum_{i=1}^{k-1} \xi_{i} \boldsymbol{n}_{i}(u)\right)
$$


with $\sum_{i=1}^{k-1} \xi_{i}^{2}=1$, where $\left\{\boldsymbol{X}, \boldsymbol{n}^{T}, \boldsymbol{n}_{1}^{S}, \ldots, \boldsymbol{n}_{k-1}^{S}\right\}$ is a pseudo-orthonormal (local) frame of $N(M)$. Without the loss of generality, we assume that $\mu \neq 0$ and $\xi_{k-1} \neq 0$. We denote that

$$
\boldsymbol{n}^{T}(u)=^{t}\left(n_{-1}^{T}(u), n_{0}^{T}(u), \ldots, n_{n}^{T}(u)\right), \boldsymbol{n}_{i}(u)=^{t}\left(n_{-1}^{i}(u), n_{0}^{i}(u), \ldots, n_{n}^{i}(u)\right) .
$$

It is enough to show that the rank of the matrix

$$
\boldsymbol{C}=\left(\begin{array}{cccc}
X_{-1} \frac{\lambda_{0}}{\lambda_{-1}}-X_{0} & -X_{-1} \frac{\lambda_{1}}{\lambda_{-1}}+X_{1} & \cdots & -X_{-1} \frac{\lambda_{n}}{\lambda_{-1}}-X_{n} \\
X_{-1 u_{1}} \frac{\lambda_{0}}{\lambda_{-1}}-X_{0 u_{1}} & -X_{-1 u_{1}} \frac{\lambda_{1}}{\lambda_{-1}}+X_{1 u_{1}} & \cdots & -X_{-1 u_{1}} \frac{\lambda_{n}}{\lambda_{-1}}-X_{n u_{1}} \\
\vdots & \vdots & \ddots & \vdots \\
X_{-1 u_{s}} \frac{\lambda_{0}}{\lambda_{-1}}-X_{0 u_{s}} & -X_{-1 u_{s}} \frac{\lambda_{1}}{\lambda_{-1}}+X_{1 u_{s}} & \cdots & -X_{-1 u_{s}} \frac{\lambda_{n}}{\lambda_{-1}}-X_{n u_{s}} \\
n_{-1}^{T} \frac{\lambda_{0}}{\lambda_{-1}}-n_{0}^{T} & -n_{-1}^{T} \frac{\lambda_{1}}{\lambda_{-1}}+n_{1}^{T} & \cdots & -n_{-1}^{T} \frac{\lambda_{n}}{\lambda_{-1}}-n_{n}^{T} \\
n_{-1}^{1} \frac{\lambda_{0}}{\lambda_{-1}}-n_{0}^{1} & -n_{-1}^{1} \frac{\lambda_{1}}{\lambda_{-1}}+n_{1}^{1} & \cdots & -n_{-1}^{1} \frac{\lambda_{n}}{\lambda_{-1}}-n_{n}^{1} \\
\vdots & \vdots & \ddots & \vdots \\
n_{-1}^{k-2} \frac{\lambda_{0}}{\lambda_{-1}}-n_{0}^{k-2} & -n_{-1}^{k-2} \frac{\lambda_{1}}{\lambda_{-1}}+n_{1}^{k-2} & \cdots & -n_{-1}^{k-2} \frac{\lambda_{n}}{\lambda_{-1}}-n_{n}^{k-2}
\end{array}\right)
$$

is $n+1$ at $(u, \lambda) \in \Sigma_{*}(H)$. We denote that

$$
\boldsymbol{a}_{i}={ }^{t}\left(x_{i}(u), x_{i u_{1}}(u), \ldots, x_{i u_{s}}(u), n_{i}^{T}(u), n_{i}^{1}(u), \ldots, n_{i}^{k-2}(u)\right) .
$$

Then, we have

$$
\boldsymbol{C}=\left(\boldsymbol{a}_{-1} \frac{\lambda_{0}}{\lambda_{-1}}-\boldsymbol{a}_{0},-\boldsymbol{a}_{-1} \frac{\lambda_{1}}{\lambda_{-1}}+\boldsymbol{a}_{1}, \ldots,-\boldsymbol{a}_{-1} \frac{\lambda_{n}}{\lambda_{-1}}+\boldsymbol{a}_{n}\right)
$$

It follows that

$$
\begin{aligned}
\operatorname{det} C & =\frac{\lambda_{-1}}{\lambda_{-1}} \operatorname{det}\left(\boldsymbol{a}_{0}, \boldsymbol{a}_{1}, \ldots, \boldsymbol{a}_{n}\right)+\frac{\lambda_{0}}{\lambda_{-1}} \operatorname{det}\left(\boldsymbol{a}_{-1} \boldsymbol{a}_{1}, \ldots, \boldsymbol{a}_{n}\right) \\
& -\frac{\lambda_{1}}{\lambda_{-1}}(-1) \operatorname{det}\left(\boldsymbol{a}_{-1}, \boldsymbol{a}_{0}, \boldsymbol{a}_{2}, \ldots, \boldsymbol{a}_{n}\right)-\cdots-\frac{\lambda_{n}}{\lambda_{-1}}(-1)^{n-1} \operatorname{det}\left(\boldsymbol{a}_{-1} \boldsymbol{a}_{0}, \boldsymbol{a}_{1}, \ldots, \boldsymbol{a}_{n-1}\right) .
\end{aligned}
$$

Moreover, we define $\delta_{i}=\operatorname{det}\left(\boldsymbol{a}_{-1}, \boldsymbol{a}_{0}, \boldsymbol{a}_{1}, \ldots, \boldsymbol{a}_{i-1}, \boldsymbol{a}_{i+1}, \ldots, \boldsymbol{a}_{n}\right)$ for $i=-1,0,1, \ldots, n$ and $\boldsymbol{a}=\left(-\delta_{-1}\right.$, $\left.-\delta_{0},-\delta_{1},(-1)^{2} \delta_{2}, \ldots,(-1)^{n-1} \delta_{n}\right)$. Then, we have

$$
\boldsymbol{a}=\boldsymbol{X} \wedge \boldsymbol{X}_{u_{1}} \wedge \cdots \wedge \boldsymbol{X}_{u_{s}} \wedge \boldsymbol{n}^{T} \wedge \boldsymbol{n}_{1} \wedge \cdots \wedge \boldsymbol{n}_{k-2}
$$

We remark that $\boldsymbol{a} \neq 0$ and $\boldsymbol{a}= \pm\|\boldsymbol{a}\| \boldsymbol{n}_{k-1}$. By the above calculation, we have

$$
\begin{aligned}
\operatorname{det} C & =\left\langle\left(\frac{\lambda-1}{\lambda_{-1}}, \frac{\lambda_{0}}{\lambda_{-1}}, \ldots, \frac{\lambda_{n}}{\lambda_{-1}}\right), \boldsymbol{a}\right\rangle=\frac{1}{\lambda_{-1}}\left\langle\boldsymbol{X}(u)+\mu\left(\boldsymbol{n}^{T}(u)+\sum_{i=1}^{k-1} \xi_{i} \boldsymbol{n}_{i}(u)\right), \boldsymbol{a}\right\rangle \\
& =\frac{1}{\lambda_{-1}} \times \pm \mu \xi_{k-1}\|\boldsymbol{a}\|= \pm \frac{\mu \xi_{k-1}\|\boldsymbol{a}\|}{\lambda_{-1}} \neq 0 .
\end{aligned}
$$

Therefore, the Jacobi matrix of $\Delta^{*} H$ is non-singular at $(u, \lambda) \in \Delta^{*} H^{-1}(0)$.

For other local coordinates of $A d S^{n+1}$, we can apply the same method for the proof as the above case. This completes the proof.

Here, we also consider the local coordinate $U_{-1}^{+}$. Since $H$ is a Morse family of hypersurfaces, we have a Legendrian immersion

$$
\mathscr{L}_{H}: \Sigma_{*}(H) \longrightarrow P T^{*}\left(A d S^{n+1}\right) \mid U_{-1}^{+}
$$

by the general theory of Legendrian singularities. By definition, we have

$$
\frac{\partial H}{\partial \lambda_{0}}(u, \lambda)=X_{-1}(u) \frac{\lambda_{0}}{\lambda_{-1}}-X_{0}(u), \frac{\partial H}{\partial \lambda_{i}}(u, \lambda)=-X_{-1}(u) \frac{\lambda_{i}}{\lambda_{-1}}+X_{i}(u),(i=1, \ldots, n) .
$$


It follows that

$$
\begin{aligned}
& {\left[\frac{\partial H}{\partial \lambda_{0}}(u, \lambda): \frac{\partial H}{\partial \lambda_{1}}(u, \lambda): \cdots: \frac{\partial H}{\partial \lambda_{n}}(u, \lambda)\right]} \\
& \quad=\left[X_{-1}(u) \lambda_{0}-X_{0}(u) \lambda_{-1}: X_{1}(u) \lambda_{-1}-X_{-1}(u) \lambda_{1}: \cdots: X_{n}(u) \lambda_{-1}-X_{-1}(u) \lambda_{n}\right] .
\end{aligned}
$$

Therefore, we have

$$
\mathscr{L}_{H}(u, \lambda)=\left(\lambda,\left[X_{-1}(u) \lambda_{0}-X_{0}(u) \lambda_{-1}: X_{1}(u) \lambda_{-1}-X_{-1}(u) \lambda_{1}: \cdots: X_{n}(u) \lambda_{-1}-X_{-1}(u) \lambda_{n}\right]\right),
$$

where

$$
\Sigma_{*}(H)=\left\{(u, \lambda) \mid \lambda=\mathbb{L} \mathbb{H}_{M}\left(\boldsymbol{n}^{T}\right)(p, \boldsymbol{\xi}, t)((p, \boldsymbol{\xi}), t) \in N_{1}(M)\left[\boldsymbol{n}^{T}\right] \times \mathbb{R}\right\} .
$$

We observe that $H$ is a generating family of the Legendrian submanifold $\mathscr{L}_{H}\left(\Sigma_{*}(H)\right)$ whose wave front is $\mathbb{L}_{M}\left(\boldsymbol{n}^{T}\right)\left(N_{1}(M)\left[\boldsymbol{n}^{T}\right] \times \mathbb{R}\right)$. Therefore we say that the AdS-height function $H$ on $M$ gives an AdS-canonical generating family for the Legendrian lift of $\mathbb{L}_{M}\left(\boldsymbol{n}^{T}\right)\left(N_{1}(M)\left[\boldsymbol{n}^{T}\right] \times \mathbb{R}\right)$. For other local coordinates of $A d S^{n+1}$, we have similar results to the above case.

\section{CONTACT WITH LIGHTCONES}

In this section, we consider the geometric meaning of the singularities of lightlike hypersurfaces in anti-de Sitter space from the view point of the theory of contact of submanifolds with model hypersurfaces in Ref. 26 . We begin with the following basic observations.

Proposition 5.1. Let $\lambda_{0} \in A d S^{n+1}$ and $M=X(U)$ a spacelike submanifold without points satisfying $K_{N}\left(\boldsymbol{n}^{T}\right)(p, \boldsymbol{\xi})=0$. Then, $M \subset \Lambda_{\lambda_{0}}^{n+1} \cap A d S^{n+1}$ if and only if $\left\{\lambda_{0}\right\}=\mathbb{L F}_{M}\left(\boldsymbol{n}^{T}\right)$ is the lightcone focal set. In this case, we have $\mathbb{L H}_{M}\left(\boldsymbol{n}^{T}\right)\left(N_{1}(M)\left[\boldsymbol{n}^{T}\right]\right) \subset \Lambda_{\lambda_{0}}^{n+1} \cap A d S^{n+1}$ and $M=\boldsymbol{X}(U)$ is totally nullcone umbilical.

Proof. By Proposition 3.1, $K_{N}\left(\boldsymbol{n}^{T}\right)\left(p_{0}, \boldsymbol{\xi}_{0}\right) \neq 0$ if and only if

$$
\left\{\left(\boldsymbol{n}^{T}+\boldsymbol{n}^{S}\right),\left(\boldsymbol{n}^{T}+\boldsymbol{n}^{S}\right)_{u_{1}}, \ldots,\left(\boldsymbol{n}^{T}+\boldsymbol{n}^{S}\right)_{u_{S}}\right\}
$$

is linearly independent for $p_{0}=\boldsymbol{X}\left(u_{0}\right) \in M$ and $\boldsymbol{\xi}_{0}=\boldsymbol{n}^{S}\left(u_{0}\right)$, where $\boldsymbol{n}^{S}: U \longrightarrow N_{1}(M)\left[\boldsymbol{n}^{T}\right]$ is a local section. By the proof of assertion (1) of Proposition 4.1, $M \subset \Lambda_{\lambda_{0}}^{n+1} \cap A d S^{n+1}$ if and only if $h_{\lambda_{0}}(u)=0$ for any $u \in U$, where $h_{\lambda_{0}}(u)=H\left(u, \lambda_{0}\right)$ is the AdS-height function on $M$. It also follows from Proposition 4.1 that there exists a smooth function $\eta: U \times N_{1}(M)\left[\boldsymbol{n}^{T}\right] \longrightarrow \mathbb{R}$ and section $\boldsymbol{n}^{S}: U \longrightarrow N_{1}(M)\left[\boldsymbol{n}^{T}\right]$ such that

$$
\boldsymbol{X}(u)=\lambda_{0}+\eta\left(u, \boldsymbol{n}^{S}(u)\right)\left(\boldsymbol{n}^{T}(u) \pm \boldsymbol{n}^{S}(u)\right) .
$$

In fact, we have $\eta\left(u, \boldsymbol{n}^{S}(u)\right)=-1 / \kappa_{N}\left(\boldsymbol{n}^{T}\right)_{i}(p, \boldsymbol{\xi}) i=1, \ldots, s$, where $p=\boldsymbol{X}(u)$ and $\boldsymbol{\xi}=\boldsymbol{n}^{S}(u)$. It follows that $\kappa_{N}\left(\boldsymbol{n}^{T}\right)_{i}(p, \boldsymbol{\xi})=\kappa_{N}\left(\boldsymbol{n}^{T}\right)_{j}(p, \boldsymbol{\xi})$, so that $M=\boldsymbol{X}(U)$ is totally nullcone umbilical. Therefore, we have

$$
\mathbb{L} \mathbb{H}_{M}\left(\boldsymbol{n}^{T}\right)\left(u, \boldsymbol{n}^{S}(u), \mu\right)=\lambda_{0}+\left(\mu+\eta\left(u, \boldsymbol{n}^{S}(u)\right)\left(\boldsymbol{n}^{T}(u) \pm \boldsymbol{n}^{S}(u)\right) .\right.
$$

Hence, we have $\mathbb{L H}_{M}\left(\boldsymbol{n}^{T}\right)\left(N_{1}(M)\left[\boldsymbol{n}^{T}\right] \times \mathbb{R}\right) \subset \Lambda_{\lambda_{0}}^{n+1}$. By Corollary 4.2, the critical value set of $\mathbb{L} \mathbb{H}_{M}\left(\boldsymbol{n}^{T}\right)\left(N_{1}(M)\left[\boldsymbol{n}^{T}\right] \times \mathbb{R}\right)$ is the lightlike focal set $\mathbb{L F}_{M}\left(\boldsymbol{n}^{T}\right)$. However, it is equal to $\lambda_{0}$ by the previous arguments.

For the converse assertion, suppose that $\lambda_{0}=\mathbb{L F}_{M}\left(\boldsymbol{n}^{T}\right)$. Then, we have

$$
\lambda_{0}=\boldsymbol{X}(u)+\frac{1}{\kappa_{N}\left(\boldsymbol{n}^{T}\right)_{i}(\boldsymbol{X}(u), \boldsymbol{\xi})} \mathbb{L} \mathbb{G}\left(\boldsymbol{n}^{T}\right)(u, \boldsymbol{\xi}),
$$

for any $i=1, \ldots, s$ and $(p, \boldsymbol{\xi}) \in N_{1}(M)\left[\boldsymbol{n}^{T}\right]$, where $p=\boldsymbol{X}(u)$. Thus, we have

$$
\kappa_{N}\left(\boldsymbol{n}^{T}\right)_{i}(\boldsymbol{X}(u), \boldsymbol{\xi})=\kappa_{N}\left(\boldsymbol{n}^{T}\right)_{j}(\boldsymbol{X}(u), \boldsymbol{\xi})
$$

for any $i, j=1, \ldots, s$, so that $M$ is totally nullcone umbilical. Since $\mathbb{L} \mathbb{G}\left(\boldsymbol{n}^{T}\right)(u, \boldsymbol{\xi})$ is null, we have $\boldsymbol{X}(u) \in \Lambda_{\lambda_{0}}^{n+1}$. This completes the proof. 
According to the above proposition, $\Lambda_{\lambda_{0}}^{n+1} \cap A d S^{n+1}$ is regarded as a model lightlike hypersurface in $A d S^{n+1}$. We define

$$
T\left(A d S^{n+1}\right) \lambda_{0}=\left\{\boldsymbol{x} \in \mathbb{R}_{2}^{n+2} \mid \boldsymbol{x}-\lambda_{0} \in T_{\lambda_{0}} A d S^{n+1}\right\},
$$

where $T_{\lambda_{0}} A d S^{n+1}$ is the tangent space of $A d S^{n+1}$ at $\lambda_{0} \in A d S^{n+1}$. We call $T\left(A d S^{n+1}\right) \lambda_{0}$ a tangent affine space of $A d S^{n+1}$ at $\lambda_{0} \in A d S^{n+1}$. It is easy to show that

$$
\Lambda_{\lambda_{0}}^{n+1} \cap A d S^{n+1}=T\left(A d S^{n+1}\right)_{\lambda_{0}} \cap A d S^{n+1} .
$$

We denote that $L C_{\lambda_{0}}\left(A d S^{n+1}\right)=\Lambda_{\lambda_{0}}^{n+1} \cap A d S^{n+1}=T\left(A d S^{n+1}\right)_{\lambda_{0}} \cap A d S^{n+1}$, which is called an $A d S$ lightcone with the vertex $\lambda_{0} \in A d S^{n+1}$. Therefore, the model lightlike hypersurface is an AdSlightcone.

We consider the contact of spacelike submanifolds with AdS-lightcones. Let

$$
\mathcal{H}: A d S^{n+1} \times A d S^{n+1} \longrightarrow \mathbb{R}
$$

be a function defined by $\mathcal{H}(\boldsymbol{x}, \boldsymbol{\lambda})=\langle\boldsymbol{x}, \boldsymbol{\lambda}\rangle+1$. Given $\lambda_{0} \in A d S^{n+1}$, we denote $\mathfrak{h}_{\lambda_{0}}(\boldsymbol{x})=\mathcal{H}\left(\boldsymbol{x}, \lambda_{0}\right)$, so that we have $\mathfrak{h}_{\lambda_{0}}^{-1}(0)=L C_{\lambda_{0}}\left(A d S^{n+1}\right)$. For any $p_{0}=\boldsymbol{X}\left(u_{0}\right) \in M, \mu_{0} \in \mathbb{R}$, and $\xi_{0} \in N_{1}(M)_{p}\left[\boldsymbol{n}^{T}\right]$, we consider the point $\lambda_{0}=\boldsymbol{X}\left(u_{0}\right)+\mu_{0}\left(\boldsymbol{n}^{T}\left(u_{0}\right)+\boldsymbol{\xi}_{0}\right)$. Then, we have

$$
\left.\mathfrak{h}_{\lambda_{0}} \circ \boldsymbol{X}\left(u_{0}\right)\right)=\mathcal{H} \circ\left(X \times 1_{A d S^{n+1}}\right)\left(u_{0}, \lambda_{0}\right)=H\left(p_{0}, \lambda_{0}\right)=0,
$$

where $\mu_{0}=1 / \kappa_{N}\left(\boldsymbol{n}^{T}\right)_{i}\left(p_{0}, \boldsymbol{\xi}_{0}\right), i=1, \ldots, s$. We also have relations

$$
\frac{\partial \mathfrak{h}_{\lambda_{0}} \circ \boldsymbol{X}}{\partial u_{i}}\left(u_{0}\right)=\frac{\partial H}{\partial u_{i}}\left(p_{0}, \lambda_{0}\right)=0, i=1, \ldots, s .
$$

These imply that the AdS-lightcone $\mathfrak{h}_{\lambda_{0}}^{-1}(0)=L C_{\lambda_{0}}\left(A d S^{n+1}\right)$ is tangent to $M=\boldsymbol{X}(U)$ at $p_{0}=\boldsymbol{X}\left(u_{0}\right)$. In this case, we call $L C_{\lambda_{0}}\left(A d S^{n+1}\right)$ a tangent $A d S$-lightcone of $M=\boldsymbol{X}(U)$ at $p_{0}=\boldsymbol{X}\left(u_{0}\right)$, which is denoted by $T L C_{\lambda_{0}}(M)_{p_{0}}$. Moreover, the tangent AdS-lightcone $T L C_{\lambda_{0}}(M)_{p_{0}}$ is called an osculating AdS-lightcone if $\lambda_{0}=\mathbb{L F}_{\kappa_{N}\left(n^{T}\right)_{i}\left(p_{0}, \xi_{0}\right)}\left(u_{0}\right) \in \mathbb{L F}_{M}$, for one of the nullcone principal curvatures $\kappa_{N}\left(\boldsymbol{n}^{T}\right)_{i}\left(p_{0}, \boldsymbol{\xi}_{0}\right)$. In this case, we call $\lambda_{0}$ the center of the nullcone principal curvature $\kappa_{N}\left(\boldsymbol{n}^{T}\right)_{i}\left(p_{0}, \boldsymbol{\xi}_{0}\right)$ $\left(u_{0}\right)$. Therefore, we can interpret that the lightlike focal set is the locus of the centers of nullcone principal curvatures. This fact is analogous to the notion of the focal sets of submanifolds in Euclidean space.

First, we consider a special contact of $M=\boldsymbol{X}(U)$ with AdS-lightcones. We say that $p_{0}=\boldsymbol{X}\left(u_{0}\right)$ is an AdS-lightlike $k$-ridge point if $h_{\lambda_{0}}$ has the $A_{k+2}$ singular point at $u_{0}$ for some $k \geq 1$, where $\lambda_{0} \in \mathcal{D}_{H}=\mathbb{L} \mathbb{H}_{M}\left(N_{1}(M) \times \mathbb{R}\right)$. We simply say that $p_{0}=\boldsymbol{X}\left(u_{0}\right)$ is an AdS-lightlike ridge point if it is the AdS-lightlike $k$-ridge point for some $k \geq 1$. For a function germ $f:\left(\mathbb{R}^{s}, \widetilde{u}_{0}\right) \longrightarrow \mathbb{R}, f$ has an $A_{k}$ singular point at $\widetilde{u}_{0}$ if $f$ is $\mathcal{K}$-equivalent to the germ $u_{1}^{k+1} \pm u_{2}^{2} \pm \cdots \pm u_{s}^{2}$. We say that two function germs $f_{i}:\left(\mathbb{R}^{s}, \widetilde{u}_{i}\right) \longrightarrow \mathbb{R}(i=1,2)$ are $\mathcal{K}$-equivalent if there exists a diffeomorphism germ $\Phi:\left(\mathbb{R}^{s}, \widetilde{u}_{1}\right) \longrightarrow\left(\mathbb{R}^{s}, \widetilde{u}_{2}\right)$ and a non-zero function germ $\rho:\left(\mathbb{R}^{s}, \widetilde{u}_{1}\right) \longrightarrow \mathbb{R}$ such that $f_{2} \circ \Phi(u)=$ $\rho(u) f_{1}(u)$. We consider the geometric meaning of AdS-lightlike ridge points. Let $F: A d S^{n+1} \longrightarrow \mathbb{R}$ be a submersion and $\boldsymbol{X}: U \longrightarrow A d S^{n+1}$ a spacelike embedding from an open set $U \subset \mathbb{R}^{s}$. We say that $M=\boldsymbol{X}(U)$ and $F^{-1}(0)$ have a corank $r$ contact at $p_{0}=\boldsymbol{X}\left(u_{0}\right)$ if the Hessian of the function $g(u)=F \circ \boldsymbol{X}(u)$ has corank $r$ at $u_{0}$. We also say that $M=\boldsymbol{X}(U)$ and $F^{-1}(0)$ have an $A_{k}$-type contact at $p_{0}=\boldsymbol{X}\left(u_{0}\right)$ if the function $g(u)=F \circ \boldsymbol{X}(u)$ has the $A_{k}$ singularity at $u_{0}$. By definition, if $M=\boldsymbol{X}(U)$ and $F^{-1}(0)$ have an $A_{k}$-type contact at $p_{0}=\boldsymbol{X}\left(u_{0}\right)$, then these have a corank 1 contact. For a regular curve $\gamma: I \longrightarrow A d S^{n+1}$, we say that $\gamma(I)$ and $F^{-1}(0)$ have a contact of order $k$ if $F \circ \gamma$ has the $A_{k}$ singularity at $s_{0}$. We have the following simple proposition.

Proposition 5.2. For any $p_{0}=X\left(u_{0}\right)$ and $\lambda_{0}=\mathbb{L F}_{\kappa_{N}\left(n^{T}\right)_{i}\left(p_{0}, \xi_{0}\right)}\left(u_{0}\right)$, there exists an integer $r$ with $1 \leq r \leq s$ such that $M=X(U)$ and $T L C_{\lambda_{0}}(M)_{p_{0}}$ have corank $r$ contact at $p_{0}=\boldsymbol{X}\left(u_{0}\right)$.

By Proposition 4.1, $M=X(U)$ and the osculating hypersphere $T L C_{\lambda_{0}}(M)_{p_{0}}$ has corank $s$ contact at an $\left(\boldsymbol{n}^{T}\left(u_{0}\right), \boldsymbol{\xi}_{0}\right)$-umbilical point. Therefore, the AdS-lightlike ridge point is not an $\left(\boldsymbol{n}^{T}\left(u_{0}\right), \boldsymbol{\xi}_{0}\right)$ umbilical point. 
By the general theory of unfoldings of function germs, the discriminant set $\mathcal{D}_{F}$ is non-singular at the origin if and only if the function $f=F \mid \mathbb{R}^{k} \times\{0\}$ has the $A_{1}$-type singularity (i.e., the Morse-type singularity). Therefore, we have the following proposition.

Proposition 5.3. With the same notations as in the previous proposition, the lightlike hypersurface $\mathbb{L} \mathbb{H}_{M}$ is non-singular at $\lambda_{0}=\mathbb{L} \mathbb{H}_{M}\left(\left(p_{0}, \xi_{0}\right), \mu_{0}\right)$ if and only if $M=X(U)$ and $T L C_{\lambda_{0}}(M)_{p_{0}}$ have the $A_{1}$-type contact at $p_{0}=\boldsymbol{X}\left(u_{0}\right)$.

We now consider the general contact of $M=X(U)$ with AdS-lightcones as an application of the theory of contact for submanifolds in Montaldi. ${ }^{26}$ Let $X_{i}$ and $Y_{i}, i=1,2$, be submanifolds of $\mathbb{R}^{n}$ with $\operatorname{dim} X_{1}=\operatorname{dim} X_{2}$ and $\operatorname{dim} Y_{1}=\operatorname{dim} Y_{2}$. We say that the contact of $X_{1}$ and $Y_{1}$ at $y_{1}$ is same type as the contact of $X_{2}$ and $Y_{2}$ at $y_{2}$ if there is a diffeomorphism germ $\Phi:\left(\mathbb{R}^{n}, y_{1}\right) \longrightarrow\left(\mathbb{R}^{n}, y_{2}\right)$ such that $\Phi\left(X_{1}\right)=X_{2}$ and $\Phi\left(Y_{1}\right)=Y_{2}$. In this case, we write $K\left(X_{1}, Y_{1} ; y_{1}\right)=K\left(X_{2}, Y_{2} ; y_{2}\right)$. Since this definition of contact is local, we can replace $\mathbb{R}^{n}$ by arbitrary $n$-manifold. Montaldi gives in Ref. 26 the following characterization of contact by using $\mathcal{K}$-equivalence.

Theorem 5.4. Let $X_{i}$ and $Y_{i}, i=1,2$, be submanifolds of $\mathbb{R}^{n}$ with $\operatorname{dim} X_{1}=\operatorname{dim} X_{2}$ and $\operatorname{dim} Y_{1}=$ $\operatorname{dim} Y_{2}$. Let $g_{i}:\left(X_{i}, x_{i}\right) \longrightarrow\left(\mathbb{R}^{n}, y_{i}\right)$ be immersion germs and $f_{i}:\left(\mathbb{R}^{n}, y_{i}\right) \longrightarrow\left(\mathbb{R}^{p}, 0\right)$ be submersion germs with $\left(Y_{i}, y_{i}\right)=\left(f_{i}^{-1}(0), y_{i}\right)$. Then,

$$
K\left(X_{1}, Y_{1} ; y_{1}\right)=K\left(X_{2}, Y_{2} ; y_{2}\right)
$$

if and only if $f_{1} \circ g_{1}$ and $f_{2} \circ g_{2}$ are $\mathcal{K}$-equivalent.

On the other hand, we now return to the review on the theory of Legendrian singularities. We introduce a natural equivalence relation among Legendrian submanifold germs. Let $F, G:\left(\mathbb{R}^{k} \times\right.$ $\left.\mathbb{R}^{n+1}, \mathbf{0}\right) \longrightarrow(\mathbb{R}, 0)$ be Morse families of hypersurfaces. Then, we say that $\mathscr{L}_{F}\left(\Sigma_{*}(F)\right)$ and $\mathscr{L}_{G}\left(\Sigma_{*}(G)\right)$ are Legendrian equivalent if there exists a contact diffeomorphism germ $H:\left(P T^{*} \mathbb{R}^{n+1}, z\right)$ $\longrightarrow\left(P T^{*} \mathbb{R}^{n+1}, z^{\prime}\right)$ such that $H$ preserves fibers of $\pi$ and that $H\left(\mathscr{L}_{F}\left(\Sigma_{*}(F)\right)\right)=\mathscr{L}_{G}\left(\Sigma_{*}(G)\right)$, where $z=\mathscr{L}_{F}(0), z^{\prime}=\mathscr{L}_{G}(0)$. By using the Legendrian equivalence, we can define the notion of Legendrian stability for Legendrian submanifold germs by the ordinary way (see, Ref. 1, Part III). We can interpret the Legendrian equivalence by using the notion of generating families. We denote $\mathcal{E}_{k}$ as the local ring of function germs $\left(\mathbb{R}^{k}, \mathbf{0}\right) \longrightarrow \mathbb{R}$ with the unique maximal ideal $\mathfrak{M}_{k}=\left\{h \in \mathcal{E}_{k} \mid h(0)=0\right\}$. Let $F, G$ : $\left(\mathbb{R}^{k} \times \mathbb{R}^{n+1}, \mathbf{0}\right) \longrightarrow(\mathbb{R}, \mathbf{0})$ be function germs. We say that $F$ and $G$ are $P$ - $\mathcal{K}$-equivalent if there exists a diffeomorphism germ $\Psi:\left(\mathbb{R}^{k} \times \mathbb{R}^{n+1}, \mathbf{0}\right) \longrightarrow\left(\mathbb{R}^{k} \times \mathbb{R}^{n+1}, \mathbf{0}\right)$ of the form $\Psi(x, u)=\left(\psi_{1}(q, x), \psi_{2}(x)\right)$ for $(q, x) \in\left(\mathbb{R}^{k} \times \mathbb{R}^{n+1}, \mathbf{0}\right)$ such that $\Psi^{*}\left(\langle F\rangle_{\mathcal{E}_{k+n+1}}\right)=\langle G\rangle_{\mathcal{E}_{k+n+1}}$. Here, $\Psi^{*}: \mathcal{E}_{k+n+1} \longrightarrow \mathcal{E}_{k+n+1}$ is the pull back $\mathbb{R}$-algebra isomorphism defined by $\Psi^{*}(h)=h \circ \Psi$. We say that $F$ is an infinitesimally $\mathcal{K}$ versal deformation of $f=F \mid \mathbb{R}^{k} \times\{\mathbf{0}\}$ if

$$
\mathcal{E}_{k}=T_{e}(\mathcal{K})(f)+\left\langle\frac{\partial F}{\partial x_{1}}\left|\mathbb{R}^{k} \times\{\mathbf{0}\}, \ldots, \frac{\partial F}{\partial x_{n+1}}\right| \mathbb{R}^{k} \times\{\mathbf{0}\}\right\rangle_{\mathbb{R}},
$$

where

$$
T_{e}(\mathcal{K})(f)=\left\langle\frac{\partial f}{\partial q_{1}}, \ldots, \frac{\partial f}{\partial q_{k}}, f\right\rangle_{\varepsilon_{k}}
$$

(see, Ref. 23). The main result in the theory of Legendrian singularities (Ref. 1, §20.8 and Ref. 32, THEOREM 2) is the following.

Theorem 5.5. Let $F, G:\left(\mathbb{R}^{k} \times \mathbb{R}^{n+1}, \mathbf{0}\right) \longrightarrow(\mathbb{R}, 0)$ be Morse families of hypersurfaces. Then, we have the following assertions:

(1) $\mathscr{L}_{F}\left(\Sigma_{*}(F)\right)$ and $\mathscr{L}_{G}\left(\Sigma_{*}(G)\right)$ are Legendrian equivalent if and only if $F$ and $G$ are $P-\mathcal{K}$ equivalent,

(2) $\mathscr{L}_{F}\left(\Sigma_{*}(F)\right)$ is Legendrian stable if and only if $F$ is an infinitesimally $\mathcal{K}$-versal deformation of $f=F \mid \mathbb{R}^{k} \times\{\mathbf{0}\}$.

Since $F$ and $G$ are function germs on the common space germ $\left(\mathbb{R}^{k} \times \mathbb{R}^{n+1}, \mathbf{0}\right)$, we do not need the notion of stably $P$-K $\mathcal{K}$-equivalences in this situation (Ref. 32, p. 27). For any map germ 
$f:\left(\mathbb{R}^{k}, \mathbf{0}\right) \longrightarrow\left(\mathbb{R}^{p}, \mathbf{0}\right)$, we define the local ring of $f$ by $Q_{r}(f)=\mathcal{E}_{k} /\left(f^{*}\left(\mathfrak{M}_{p}\right) \mathcal{E}_{n}+\mathfrak{M}_{k}^{r+1}\right)$. We have the following classification result of Legendrian stable germs (cf. Ref. 13, Proposition A.4) which is the key for the purpose in this section.

Proposition 5.6. Let $F, G:\left(\mathbb{R}^{k} \times \mathbb{R}^{n+1}, \mathbf{0}\right) \longrightarrow(\mathbb{R}, 0)$ be Morse families of hypersurfaces and $f=F\left|\mathbb{R}^{k} \times\{\mathbf{0}\}, g=G\right| \mathbb{R}^{k} \times\{\mathbf{0}\}$. Suppose that $\mathscr{L}_{F}\left(\Sigma_{*}(F)\right)$ and $\mathscr{L}_{G}\left(\Sigma_{*}(G)\right)$ are Legendrian stable. The following conditions are equivalent:

(1) $\left(W\left(\mathscr{L}_{F}\right), \mathbf{0}\right)$ and $\left(W\left(\mathscr{L}_{G}\right), \mathbf{0}\right)$ are diffeomorphic as set germs,

(2) $\mathscr{L}_{F}\left(\Sigma_{*}(F)\right)$ and $\mathscr{L}_{G}\left(\Sigma_{*}(G)\right)$ are Legendrian equivalent,

(3) $Q_{n+2}(f)$ and $Q_{n+2}(g)$ are isomorphic as $\mathbb{R}$-algebras.

We now describe the contacts of spacelike submanifolds in $A d S^{n+1}$ with AdS-lightcones. We denote $Q\left(\boldsymbol{X}, u_{0}\right)$ as the local ring of the function germ $\widetilde{h}_{\lambda_{0}}:\left(U, u_{0}\right) \longrightarrow \mathbb{R}$, where $\lambda_{0}=\mathbb{L} \mathbb{C}_{M}\left(u_{0}, \boldsymbol{\xi}_{0}, \mu_{0}\right)$. We remark that we can explicitly write the local ring as follows:

$$
Q_{n+2}\left(X, u_{0}\right)=\frac{C_{u_{0}}^{\infty}(U)}{\left\langle\left\langle X(u), \lambda_{0}\right\rangle+1\right\rangle_{C_{u_{0}}^{\infty}(U)}+\mathfrak{M}_{u_{0}}(U)^{n+2}},
$$

where $C_{u_{0}}^{\infty}(U)$ is the local ring of function germs on $U$ at $u_{0}$.

Let $\mathbb{L H}_{M_{i}}\left(\boldsymbol{n}_{i}^{T}\right):\left(N_{1}\left(M_{i}\right)\left[\boldsymbol{n}_{i}^{T}\right] \times \mathbb{R},\left(p_{i}, \boldsymbol{\xi}_{i}, \mu_{i}\right)\right) \longrightarrow\left(A d S^{n+1}, \lambda_{i}\right),(i=1,2)$ be two lightlike hypersurface germs of spacelike submanifold germs $\boldsymbol{X}_{i}:\left(U, u^{i}\right) \longrightarrow\left(A d S^{n+1}, p_{i}\right)$. Let $H_{i}:\left(U \times A d S^{n+1}\right.$, $\left.\left(u^{i}, \lambda_{i}\right)\right) \longrightarrow \mathbb{R}$ be the AdS-height function germ of $\boldsymbol{X}_{i}$. Then, we have the following theorem.

Theorem 5.7. Let $\boldsymbol{X}_{i}:\left(U, u^{i}\right) \longrightarrow\left(A d S^{n+1}, p_{i}\right), i=1,2$, be spacelike submanifold germs such that the corresponding Legendrian submanifold germs $\mathscr{L}_{H_{i}}\left(\Sigma_{*}\left(H_{i}\right)\right)$ are Legendrian stable. We denote that $\boldsymbol{X}_{i}(U)=M_{i}$. Then, the following conditions are equivalent:

(1) $\left(\mathbb{L} \mathbb{H}_{M_{1}}\left(N_{1}\left(M_{1}\right)\left[\boldsymbol{n}_{1}^{T}\right] \times \mathbb{R}\right), \lambda_{1}\right)$ and $\left(\mathbb{L} \mathbb{H}_{M_{2}}\left(N_{1}\left(M_{2}\right)\left[\boldsymbol{n}_{2}^{T}\right] \times \mathbb{R}\right), \lambda_{2}\right)$ are diffeomorphic,

(2) $\left(\mathscr{L}_{H_{1}}\left(\Sigma_{*}\left(H_{1}\right)\right),\left(u^{1}, \lambda_{1}\right)\right)$ and $\left(\mathscr{L}_{H_{2}}\left(\Sigma_{*}\left(H_{2}\right)\right),\left(u^{2}, \lambda_{2}\right)\right)$ are Legendrian equivalent,

(3) $H_{1}$ and $H_{2}$ are $P$ - $\mathcal{K}$-equivalent,

(4) $h_{1, \lambda_{1}}$ and $h_{2, \lambda_{2}}$ are $\mathcal{K}$-equivalent,

(5) $K\left(M_{1}, T L C_{\lambda_{1}}\left(M_{1}\right)_{p_{1}}, p_{1}\right)=K\left(M_{2}, T L C_{\lambda_{2}}\left(M_{2}\right)_{p_{2}}, p_{2}\right)$,

(6) $\quad Q_{n+1}\left(\boldsymbol{X}_{1}, u^{1}\right)$ and $Q_{n+1}\left(\boldsymbol{X}_{2}, u^{2}\right)$ are isomorphic as $\mathbb{R}$-algebras.

Proof. By Proposition 5.6, conditions (1), (2), and (6) are equivalent. This condition is also equivalent to that two generating families $H_{1}$ and $H_{2}$ are $P-\mathcal{K}$-equivalent by Theorem 5.3. If we denote $h_{i, \lambda_{i}}(u)=H_{i}\left(u, \lambda_{i}\right)$, then we have $h_{i, \lambda_{i}}(u)=\mathfrak{h}_{\lambda_{i}} \circ \boldsymbol{X}_{i}(u)$. By Theorem 5.2, $K\left(\boldsymbol{X}_{1}(U), L C_{\lambda_{1}}, p_{1}\right)$ $=K\left(\boldsymbol{x}_{2}(U), L C \lambda_{2}, p_{2}\right)$ if and only if $\widetilde{h}_{1, \lambda_{1}}$ and $\widetilde{h}_{2, \lambda_{2}}$ are $\mathcal{K}$-equivalent. This means that (4) and (5) are equivalent. By definition, (3) implies (4). The uniqueness of the infinitesimally $\mathcal{K}$-versal deformation of $h_{i, \lambda_{i}}$ (cf. Ref. 23) leads that (4) implies (3). This completes the proof.

For a spacelike embedding germ $\boldsymbol{X}:\left(U, u_{0}\right) \longrightarrow\left(A d S^{n+1}, p_{0}\right)$, we consider a set germ $\left(\boldsymbol{X}^{-1}\right.$ $\left.\left(T L C_{\lambda_{0}}(M)_{p_{0}}\right), u_{0}\right)$, which is called the AdS-tangent lightcone indicatrix germ of $\boldsymbol{X}$, where $\lambda_{0}=$ $\mathbb{L} \mathbb{H}_{M}\left(p_{0}, \boldsymbol{\xi}_{0}, \mu_{0}\right)$ and $\mu_{0}=-1 / \kappa_{N}\left(\boldsymbol{n}^{T}\right)_{i}\left(p_{0}, \boldsymbol{\xi}_{0}\right)(i=1, \ldots, s)$. We have the following corollary of Theorem 5.7 .

Corollary 5.8. With the assumptions of Theorem 5.7, if the lightlike hypersurface germs

$$
\left(\mathbb{L} \mathbb{H}_{M_{1}}\left(N_{1}\left(M_{1}\right)\left[\boldsymbol{n}_{1}^{T}\right] \times \mathbb{R}\right), \lambda_{1}\right) \text { and }\left(\mathbb{L H}_{M_{2}}\left(N_{1}\left(M_{2}\right)\left[\boldsymbol{n}_{2}^{T}\right] \times \mathbb{R}\right), \lambda_{2}\right)
$$

are diffeomorphic as set germs, then AdS-tangent lightcone indicatrix germs

$$
\left(\boldsymbol{X}_{1}^{-1}\left(T L C_{\lambda_{1}}\left(M_{1}\right)\right)_{p_{1}}, u^{1}\right) \text { and }\left(\boldsymbol{X}_{2}^{-1}\left(T L C_{\lambda_{2}}\left(M_{2}\right)_{p_{2}}\right), u^{2}\right)
$$

are diffeomorphic as set germs.

Proof. We remark that the tangent lightcone indicatrix germ of $\boldsymbol{X}_{i}$ is the zero level set of $h_{i, \lambda_{i}}$. Since $\mathcal{K}$-equivalence among function germs preserves the zero-level sets of function germs, the assertion follows from Theorem 5.7. 
On the other hand, we consider generic properties of lightlike hypersurfaces along spacelike submanifolds. Let $\operatorname{Emb}_{\mathrm{sp}}\left(U, A d S^{n+1}\right)$ be the space of spacelike embeddings with the $C^{\infty}$-topology for an open set $U \subset \mathbb{R}^{s}$. We consider the function $\mathcal{H}: A d S^{n+1} \times A d S^{n+1} \longrightarrow \mathbb{R}$ again. We claim that $\mathfrak{h}_{\lambda}$ is a submersion at $\boldsymbol{x} \neq \lambda$ for any $\lambda \in A d S^{n+1}$. For any $\boldsymbol{X} \in \operatorname{Emb}_{\mathrm{sp}}\left(U, A d S^{n+1}\right)$, we have $H=\mathcal{H} \circ\left(X \times 1_{A d S^{n+1}}\right)$. We have the $r$-jet extension $j_{1}^{r} H: U \times A d S^{n+1} \longrightarrow J^{r}(U, \mathbb{R})$ defined by $j_{1}^{r} H(u, \lambda)=j^{r} h_{\lambda}(u)$, where $J^{k}(U, \mathbb{R})$ is the $k$-jet space of functions on $U$. We consider the trivialization $J^{r}(U, \mathbb{R}) \equiv U \times \mathbb{R} \times J^{r}(s, 1)$. For any submanifold $Q \subset J^{r}(s, 1)$, we denote that $\widetilde{Q}=U \times \mathbb{R} \times Q$. As an application of Ref. 30 [Lemma 6], the set

$$
T_{Q}=\left\{\boldsymbol{X} \in \operatorname{Emb}_{\mathrm{sp}}\left(U, A d S^{n+1}\right) \mid j_{1}^{r} G \text { is transversal to } \widetilde{Q}\right\}
$$

is a residual set of $\operatorname{Emb}_{\mathrm{sp}}\left(U, A d S^{n+1}\right)$. Moreover, if $Q$ is a closet subset, then $T_{Q}$ is open. It is known ${ }^{6}$ that there exists a semi-algebraic set $W^{r}(s, 1) \subset J^{k}(s, 1)$ and a stratification $\mathcal{A}^{r}(s, 1)$ of $J^{k}(s, 1) \backslash W^{r}(s, 1)$ such that $\lim _{k \mapsto \infty} \operatorname{cod} W^{r}(s, 1)=+\infty$. The stratification $\mathcal{A}^{r}(s, 1)$ is called the canonical stratification. We define a stratification $\mathcal{A}^{r}(U, \mathbb{R})$ of $J^{r}(U, \mathbb{R}) \backslash W^{r}(U, \mathbb{R})$ by

$$
U \times(\mathbb{R} \backslash\{0\}) \times\left(J^{r}(s, 1) \backslash W^{r}(s, 1)\right), U \times\{0\} \times \mathcal{A}^{r}(s, 1),
$$

where $W^{r}(U, \mathbb{R})=U \times \mathbb{R} \times W^{r}(s, 1)$. In Ref. 29, it was shown that if $j_{1}^{r} H\left(U \times A d S^{n+1}\right) \cap W^{r}(U, \mathbb{R})=$ $\emptyset$ and $j_{1}^{r} H$ is transversal to $\mathcal{A}^{r}(U, \mathbb{R})$, then the map $\pi \mid H^{-1}(0): H^{-1}(0) \longrightarrow A d S^{n+1}$ is MT-stable mapgerm at each point, where $\pi: U \times \mathbb{R}_{1}^{n+1} \longrightarrow A d S^{n+1}$ is the canonical projection. Here, a map germ is said to be MT-stable if the jet extension is transversal to the canonical stratification of the jet space of sufficiently higher order (cf. Refs. 6 and 24). The main result of the theory of topological stability of Mather is that MT-stability implies topological stability. By Proposition 4.1, the lightlike hypersurface $\mathbb{L H}_{M}\left(\boldsymbol{n}^{T}\right)\left(N_{1}(M)\left[\boldsymbol{n}^{T}\right] \times \mathbb{R}\right)$ is the discriminant set of $H$, which is equal to the critical value set of $\pi \mid H^{-1}(0)$. Since $\operatorname{cod} W^{r}(U, \mathbb{R})>s+n+1$ for sufficiently large $k$, the set

$$
O_{1}=\left\{\boldsymbol{X} \in \operatorname{Emb}_{\mathrm{sp}}\left(U, A d S_{1}^{n+1}\right) \mid j_{1}^{r} H\left(U \times A d S^{n+1}\right) \cap W^{r}(U, \mathbb{R})=\emptyset\right\}
$$

is a residual set. It follows that the set

$$
O=\left\{\boldsymbol{X} \in O_{1} \mid j_{1}^{r} H \text { is transversal to } \mathcal{A}^{r}(U, \mathbb{R})\right\}
$$

is a residual set. Therefore, we have the following theorem.

Theorem 5.9. There exists a residual set $O \subset \operatorname{Emb}_{\mathrm{sp}}\left(U, A d S^{n+1}\right)$ such that for any $\boldsymbol{X} \in O$, the germ of the lightlike hypersurface $\mathbb{L} \mathbb{H} M_{M}\left(\boldsymbol{n}^{T}\right)\left(N_{1}(M)\left[\boldsymbol{n}^{T}\right] \times \mathbb{R}\right)$ at any point is a germ of the critical value set of a MT-stable map germ.

In the case when $n \leq 5$, by the classification results of the $\mathcal{K}$-equivalence among function germs, the canonical stratification $\mathcal{A}^{k}(s, 1)$ is given by the finite collection of the $\mathcal{K}$-orbits. Moreover, if $j_{1}^{r} H$ is transversal to the $\mathcal{K}$-orbit of $j^{r} h_{\lambda_{0}}\left(u_{0}\right)$ for sufficiently large $r$, then $H$ is an infinitesimally $\mathcal{K}$-versal deformation of $h_{\lambda}$ at $\left(u_{0}, \lambda_{0}\right) .{ }^{23}$ By Theorem 5.5, we have the following theorem.

Theorem 5.10. Suppose that $n \leq 5$. Then, there exists a residual set $O \subset \operatorname{Emb}_{\mathrm{sp}}\left(U, A d S^{n+1}\right)$ such that for any $\boldsymbol{X} \in O$, the germ of the lightlike hypersurface $\mathbb{L} \mathbb{H}_{M}\left(\boldsymbol{n}^{T}\right)\left(N_{1}(M)\left[\boldsymbol{n}^{T}\right] \times \mathbb{R}\right)$ at any point is the germ of the wave front set of a stable Legendrian submanifold germ $\mathscr{L}_{H}\left(\Sigma_{*}(H)\right)$.

\section{SPACELIKE SUBMANIFOLDS WITH CODIMENSION TWO}

In the case when $s=n-1, N_{1}(M)\left[\boldsymbol{n}^{T}\right]$ is a double covering of $M=\boldsymbol{X}(U)$. We can construct a spacelike unit normal section $\boldsymbol{n}^{S}(u) \in N_{p}(M)$ by

$$
\boldsymbol{n}^{S}(u)=\frac{\boldsymbol{X}(u) \wedge \boldsymbol{n}^{T}(u) \wedge \boldsymbol{X}_{u_{1}}(u) \wedge \cdots \wedge \boldsymbol{X}_{u_{n-1}}(u)}{\left\|\boldsymbol{X}(u) \wedge \boldsymbol{n}^{T}(u) \wedge \boldsymbol{X}_{u_{1}}(u) \wedge \cdots \wedge \boldsymbol{X}_{u_{n-1}}(u)\right\|} .
$$

Then, $\boldsymbol{\sigma}^{ \pm}(u)=\left(\boldsymbol{X}(u), \pm \boldsymbol{n}^{S}(u)\right)$ are sections of $N_{1}(M)\left[\boldsymbol{n}^{T}\right]$. We call $\left(\boldsymbol{n}^{T}, \boldsymbol{n}^{S}\right)$ an adopted normal frame along $M=\boldsymbol{X}(U)$. The vectors $\boldsymbol{n}^{T}(u) \pm \boldsymbol{n}^{S}(u)$ are null. Since $\left\{\boldsymbol{X}_{u_{1}}(u), \ldots, \boldsymbol{X}_{u_{n-1}}(u)\right\}$ is a basis of $T_{p} M$, the system $\left\{\boldsymbol{X}(u), \boldsymbol{n}^{T}(u), \boldsymbol{n}^{S}(u), \boldsymbol{X}_{u_{1}}(u), \ldots, \boldsymbol{X}_{u_{n-1}}(u)\right\}$ provides a basis for $T_{p} \mathbb{R}_{2}^{n+2}$ such that $\left\{\boldsymbol{n}^{T}(u), \boldsymbol{n}^{S}(u)\right\}$ is a pseudo-orthonormal frame of the normal timelike plane $N_{p}(M) \cap T_{p} A d S^{n+1}$ in $A d S^{n+1}$. 
Lemma 6.1. Given two adopted unit timelike normal sections $\boldsymbol{n}^{T}(u), \overline{\boldsymbol{n}}^{T}(u) \in N_{p}(M)$, the corresponding nullcone normal sections $\boldsymbol{n}^{T}(u) \pm \boldsymbol{n}^{S}(u), \overline{\boldsymbol{n}}^{T}(u) \pm \overline{\boldsymbol{n}}^{S}(u)$ are parallel.

Proof. We consider the orientation and the timelike orientation on the normal space $N_{p}(M)$ induced by the orientation and the timelike orientation of $\mathbb{R}_{1}^{n+1}$ and $\left\{\boldsymbol{X}_{u_{1}}(u), \ldots, \boldsymbol{X}_{u_{n-1}}(u)\right\}$. By the construction, both the pseudo-orthogonal bases $\left\{\boldsymbol{n}^{T}(u), \boldsymbol{n}^{S}(u)\right\}$ and $\left\{\overline{\boldsymbol{n}}^{T}(u), \overline{\boldsymbol{n}}^{S}(u)\right\}$ of $N_{p}(M) \cap$ $T_{p} A d S^{n}$ correspond to the same orientation and the same timelike orientation on $N_{p}(M) \cap T_{p} A d S^{n}$. Since both $\boldsymbol{n}^{T}(u)$ and $\overline{\boldsymbol{n}}^{T}(u)$ are adopted and $\boldsymbol{n}^{T}(u) \pm \boldsymbol{n}^{S}(u), \overline{\boldsymbol{n}}^{T}(u) \pm \overline{\boldsymbol{n}}^{S}(u)$ are null in the Lorentz plane $N_{p}(M) \cap T_{p} A d S^{n+1}, \boldsymbol{n}^{T}(u) \pm \boldsymbol{n}^{S}(u)$ and $\overline{\boldsymbol{n}}^{T}(u) \pm \overline{\boldsymbol{n}}^{S}(u)$ are parallel. This completes the proof.

Therefore, the null cone Gauss images of $M=X(U)$ with respect to $\left(\boldsymbol{n}^{T}, n^{S}\right)$ are given by $\mathbb{N} G\left(\boldsymbol{n}^{T}, \pm \boldsymbol{n}^{S}\right)(u)=\boldsymbol{n}^{T}(u) \pm \boldsymbol{n}^{S}(u)$. Since $N_{p}(M)\left[\boldsymbol{n}^{T}\right]$ is a spacelike line in $N_{p}(M)$, we have $\boldsymbol{\xi}=$ $\boldsymbol{n}^{S}(u)$ or $\boldsymbol{\xi}=-\boldsymbol{n}^{S}(u)$ for any $(\boldsymbol{X}(u), \boldsymbol{\xi}) \in N_{1}(M)\left[\boldsymbol{n}^{T}\right]$. We denote that $\kappa_{i}\left(\boldsymbol{n}^{T}, \pm \boldsymbol{n}^{S}\right)(u), i=1, \ldots, n-1$ instead of $\kappa_{N}\left(\boldsymbol{n}^{T}\right)_{i}(p, \boldsymbol{\xi}), i=1, \ldots, n-1$ for $\boldsymbol{\xi}= \pm \boldsymbol{n}^{S}(u)$ and $p=\boldsymbol{X}(u)$. Then, we have the following decomposition of the lightlike hypersurface along $M=\boldsymbol{X}(U)$ :

$$
\mathbb{L} \mathbb{H}_{M}\left(N_{1}(M)\left[\boldsymbol{n}^{T}\right] \times \mathbb{R}\right)=\mathbb{L} \mathbb{H}_{M}^{+}(U \times \mathbb{R}) \cup \mathbb{L} \mathbb{H}_{M}^{-}(U \times \mathbb{R}),
$$

where

$$
\mathbb{L} \mathbb{H}_{M}^{ \pm}(u, \mu)=\boldsymbol{X}(u)+\mu\left(\boldsymbol{n}^{T} \pm \boldsymbol{n}^{S}\right)(u) .
$$

In this case, the critical value of $\mathbb{L} \mathbb{H}_{M}^{ \pm}$is the point where $\kappa_{i}\left(\boldsymbol{n}^{T}, \pm \boldsymbol{n}^{S}\right)(p) \neq 0$ and

$$
\lambda^{ \pm}=\boldsymbol{X}(u)+\frac{1}{\kappa_{i}\left(\boldsymbol{n}^{T}, \pm \boldsymbol{n}^{S}\right)(u)}\left(\boldsymbol{n}^{T} \pm \boldsymbol{n}^{S}\right)(u) .
$$

For each $i=1, \ldots, n-1$, we have a mapping $\mathbb{L E}_{\kappa_{i}\left(n^{T}, \pm n^{S}\right)}: O_{i} \longrightarrow A d S^{n+1}$ defined by

$$
\mathbb{L} \mathbb{E}_{\kappa_{i}\left(\boldsymbol{n}^{T}, \pm \boldsymbol{n}^{S}\right)}(u)=\boldsymbol{X}(u)+\frac{1}{\kappa_{i}\left(\boldsymbol{n}^{T}, \pm \boldsymbol{n}^{S}\right)(u)}\left(\boldsymbol{n}^{T} \pm \boldsymbol{n}^{S}\right)(u),
$$

where $O_{i}=\left\{u \in U \mid \kappa_{i}\left(\boldsymbol{n}^{T}, \pm \boldsymbol{n}^{S}\right)(u) \neq 0\right\}$. Then, we define

$$
\mathbb{L} \mathbb{E}_{M}^{ \pm}=\bigcup\left\{\mathbb{L E}_{\kappa_{i}\left(\boldsymbol{n}^{T}, \pm \boldsymbol{n}^{S}\right)}(u) \mid u \in U \text { such that } \kappa_{i}\left(\boldsymbol{n}^{T}, \pm \boldsymbol{n}^{S}\right)(u) \neq 0, i=1, \ldots, n-1 .\right\} .
$$

By the above arguments, we know that $\mathbb{L} \mathbb{E}_{M}^{ \pm}$is nothing but the AdS-lightlike focal set of $M=X(U)$ relative to $\boldsymbol{n}^{T}$. However, we call it the AdS-lightlike evolute of $M=X(U)$ in the case when $\operatorname{codim} M=2$. For any $p_{0}=\boldsymbol{X}\left(u_{0}\right)$, we have the tangent AdS-lightcones $T L C_{\boldsymbol{\lambda}_{0}}^{ \pm}(M)_{p_{0}}$, where $\boldsymbol{\lambda}_{0}^{ \pm}=$ $\mathbb{L E}_{\kappa_{i}\left(\boldsymbol{n}^{T}, \pm \boldsymbol{n}^{S}\right)}\left(u_{0}\right)$. In the general codimension case, it depends on the choice of $\boldsymbol{n}^{S}$, so that there are infinitely many tangent AdS-lightcones of $M$ at $p_{0}=\boldsymbol{X}\left(u_{0}\right)$. However, the codimension two case, $\boldsymbol{n}^{S}$ is uniquely determined by $\boldsymbol{n}^{T}$. Therefore, we have two tangent AdS-lightcones of $M$ at $p_{0}=\boldsymbol{X}\left(u_{0}\right)$. In this case, each one of the tangent AdS-lightcones $T L C_{\lambda_{0}}^{ \pm}(M)_{p_{0}}$ is called an osculating AdS-lightcone of $M=\boldsymbol{x}(U)$. The AdS-lightlike evolutes are the loci of the vertices of osculating AdS-lightcones of $M$. Analogous to the case of hypersurfaces in Euclidean space, we call $\mathbb{L} \mathbb{E}_{M}^{ \pm}$the AdS-lightlike evolute of $M=X(U)$.

We now consider the low dimension cases in Subsections VI A and VI B.

\section{A. Spacelike curves in $A d S^{3}$}

We consider spacelike curves in $A d S^{3}$ as a simplest case of the codimension two case. Let $\gamma: I \longrightarrow A d S^{3}$ be a unit speed spacelike curve with $\left\langle\gamma^{\prime \prime}(s), \gamma^{\prime \prime}(s)\right\rangle \neq-1$, where $I$ is an open interval. We denote that $C=\gamma(I)$. Then, we define $\boldsymbol{t}(s)=\gamma^{\prime}(s)$ and call $\boldsymbol{t}(s)$ a unit tangent vector of $\gamma$ at $s$. The curvature of $\gamma$ at $s$ is defined to be $\kappa_{g}(s)=\sqrt{\left|\left\langle\gamma(s)-\gamma^{\prime \prime}(s), \gamma(s)-\gamma^{\prime \prime}(s)\right\rangle\right|}$. Since $\kappa_{g}(s) \neq 0$, the unit principal normal vector $\boldsymbol{n}(s)$ of the curve $\gamma$ at $s$ is defined by $\gamma^{\prime \prime}(s)-\gamma(s)=$ $\kappa_{g}(s) \boldsymbol{n}(s)$. We denote that $\delta(\boldsymbol{\gamma}(s))=\operatorname{sign}(\boldsymbol{n}(s))$. The unit vector $\boldsymbol{b}(s)=\boldsymbol{\gamma}(s) \wedge \boldsymbol{t}(s) \wedge \boldsymbol{n}(s)$ is called a unit binormal vector of the curve $\gamma$ at $s$. Since $\gamma(s)$ is timelike and $t(s)$ is spacelike, we have 
$\langle\boldsymbol{b}(s), \boldsymbol{b}(s)\rangle=-\delta(\boldsymbol{\gamma}(s))$ and $\operatorname{sign}\left(\boldsymbol{\gamma}^{\prime}(s)\right)=1$. Then, the following Frenet-Serret type formulae hold

$$
\left\{\begin{array}{l}
\boldsymbol{\gamma}^{\prime}(s)=\boldsymbol{t}(s), \\
\boldsymbol{t}^{\prime}(s)=\kappa_{g}(s) \boldsymbol{n}(s)+\gamma(s), \\
\boldsymbol{n}^{\prime}(s)=\delta(\boldsymbol{\gamma}(s))\left(-\kappa_{g}(s) \boldsymbol{t}(s)+\tau_{g}(s) \boldsymbol{b}(s)\right), \\
\boldsymbol{b}^{\prime}(s)=\delta(\gamma(s)) \tau_{g}(s) \boldsymbol{n}(s),
\end{array}\right.
$$

where $\tau_{g}(s)=\left\langle\boldsymbol{b}^{\prime}(s), \boldsymbol{n}(s)\right\rangle$ is the torsion of the curve $\boldsymbol{\gamma}$ at $s$. In Ref. 5, the singularities of lightlike hypersurfaces along spacelike curves in $A d S^{3}$ are classified. We define an invariant $\sigma^{ \pm}(s)=$ $\kappa_{g}^{\prime}(s) \mp \kappa_{g}(s) \tau_{g}(s)$. Then, we have the following theorem.

Theorem 6.2 (Ref. 5). Let $\gamma: I \longrightarrow A d S^{3}$ be a unit speed spacelike curve with $\left\langle\gamma^{\prime \prime}(s), \gamma^{\prime \prime}(s)\right\rangle$ $\neq-1$. Then, we have the following:

(1) The AdS-lightlike evolute of $\gamma$ is

$$
\mathbb{L E}_{C}=\left\{\lambda=\boldsymbol{\gamma}(s)+\frac{1}{\delta(\boldsymbol{\gamma}(s)) \kappa_{g}(s)}(\boldsymbol{n}(s) \pm \boldsymbol{b}(s))\right\} .
$$

(2) The germ of $\mathbb{L}_{\mathbb{H}_{M}}$ at $\lambda_{0}=\mathbb{L} \mathbb{E}_{\kappa_{g}}\left(s_{0}\right)$ is diffeomorphic to the cuspidal edge $C(2,3) \times \mathbb{R}$ if and only if $\sigma^{ \pm}\left(s_{0}\right) \neq 0$.

(3) The germ of $\mathbb{L} \mathbb{H} \mathbb{H}_{M}$ at $\lambda_{0}=\mathbb{L} \mathbb{E}_{\kappa_{g}}\left(s_{0}\right)$ is diffeomorphic to the swallowtail $S W$ if and only if $\sigma^{ \pm}\left(s_{0}\right)=0$ and $\left(\sigma^{ \pm}\right)^{\prime}\left(s_{0}\right) \neq 0$.

Here, $C(2,3) \times \mathbb{R}=\left\{\left(x_{1}, x_{2}\right) \mid x_{1}^{2}-x_{2}^{3}=0\right\} \times \mathbb{R}$ is the cuspidal edge and $S W=\left\{\left(x_{1}, x_{2}, x_{3}\right) \mid x_{1}\right.$ $\left.=3 u^{2}+u^{2} v, x_{2}=4 u^{3}+2 u v, x_{3}=v,(u, v) \in\left(\mathbb{R}^{2}, 0\right)\right\}$ is the swallowtail.

Also shown have been the following geometric characterizations of the singularities of AdSlightlike hypersurfaces in Ref. 5.

Proposition 6.3. Let $\gamma: I \longrightarrow A d S^{3}$ be a unit speed spacelike curve with $\left\langle\gamma^{\prime \prime}(s), \gamma^{\prime \prime}(s)\right\rangle \neq-1$. Then, we have the following:

(1) $\kappa_{g}\left(s_{0}\right) \neq 0$ and $\sigma^{ \pm}\left(s_{0}\right) \neq 0$ if and only if $C=\gamma(I)$ and the osculating AdS-lightcone TLC $C_{\lambda_{0}}^{ \pm}$ $(C)_{p_{0}}$ have contact of order 2 .

(2) $\kappa_{g}\left(s_{0}\right) \neq 0, \sigma^{ \pm}\left(s_{0}\right)=0$, and $\left(\sigma^{ \pm}\right)^{\prime}\left(s_{0}\right) \neq 0$ if and only if $C=\gamma(I)$ and the osculating AdSlightcone $T L C_{\lambda_{0}}^{ \pm}(C)_{p_{0}}$ have contact of order 3 .

\section{B. Spacelike surfaces in $A d S^{4}$}

We consider spacelike surfaces in $A d S^{4}$ here. Let $\boldsymbol{X}: U \longrightarrow A d S^{4}$ be a spacelike embedding from an open subset $U \subset \mathbb{R}^{2}$. As a corollary of Theorem 5.10, we have the following generic classification theorem. We say that two map germs $f, g:\left(\mathbb{R}^{n}, 0\right) \longrightarrow\left(\mathbb{R}^{p}, 0\right)$ are $\mathcal{A}$-equivalent if there exist diffeomorphism germs $\phi:\left(\mathbb{R}^{n}, 0\right) \longrightarrow\left(\mathbb{R}^{n}, 0\right)$ and $\psi:\left(\mathbb{R}^{p}, 0\right) \longrightarrow\left(\mathbb{R}^{p}, 0\right)$ such that $f \circ \phi=\psi \circ g$.

Theorem 6.4. There exists an open dense subset $O \subset \operatorname{Emb}_{\mathrm{sp}}\left(U, A d S^{4}\right)$ such that for any $\boldsymbol{X} \in$ $O$, the germ of the corresponding lightlike hypersurfaces $\mathbb{L} \mathbb{H}_{M}^{ \pm}$at any point $\left(u_{0}, \mu_{0}\right) \in U \times \mathbb{R}$ is $\mathcal{A}$-equivalent to one of the map germs $A_{k}(1 \leq k \leq 4)$ or $D_{4}^{ \pm}$: where, $A_{k}, D_{4}^{ \pm}$-map germ $f$ : $\left(\mathbb{R}^{3}, 0\right) \longrightarrow\left(\mathbb{R}^{4}, 0\right)$ are given by

$$
\begin{aligned}
& A_{1}: f\left(u_{1}, u_{2}, u_{3}\right)=\left(u_{1}, u_{2}, u_{3}, 0\right), \\
& A_{2}: f\left(u_{1}, u_{2}, u_{3}\right)=\left(3 u_{1}^{2}, 2 u_{1}^{3}, u_{2}, u_{3}\right), \\
& A_{3}: f\left(u_{1}, u_{2}, u_{3}\right)=\left(4 u_{1}^{3}+2 u_{1} u_{2}, 3 u_{1}^{4}+u_{2} u_{1}^{2}, u_{2}, u_{3}\right), \\
& A_{4}: f\left(u_{1}, u_{2}, u_{3}\right)=\left(5 u_{1}^{4}+3 u_{2} u_{1}^{2}+2 u_{1} u_{3}, 4 u_{1}^{5}+2 u_{2} u_{1}^{3}+u_{3} u_{1}^{2}, u_{2}, u_{3}\right), \\
& D_{4}^{+}: f\left(u_{1}, u_{2}, u_{3}\right)=\left(2\left(u_{1}^{3}+u_{2}^{3}\right)+u_{1} u_{2} u_{3}, 3 u_{1}^{2}+u_{2} u_{3}, 3 u_{2}^{2}+u_{1} u_{3}, u_{3}\right), \\
& D_{4}^{-}: f\left(u_{1}, u_{2}, u_{3}\right)=\left(\left(\frac{u_{1}^{3}}{3}-u_{1} u_{2}^{2}\right)+\left(u_{1}^{2}+u_{2}^{2}\right) u_{3}, u_{2}^{2}-u_{1}^{2}-2 u_{1} u_{3}, 2\left(u_{1} u_{2}-u_{2} u_{3}\right), u_{3}\right) .
\end{aligned}
$$


Proof. By Theorems 5.5 and 5.10, the AdS-height function $H$ on $M$ is a $\mathcal{K}$-versal deformation of $h_{\lambda_{0}}$ at each $\left(u_{0}, \lambda_{0}\right) \in U \times A d S^{4}$. Therefore, we can apply the classification of $\mathcal{K}$-versal deformations $F(x, y, \lambda)$ of function germs up to 4-parameters. ${ }^{1}$ For any $F(x, y, \lambda)$, we have

$$
\Sigma_{*}(F)=\left\{(x, y, \lambda) \in\left(\mathbb{R}^{2} \times \mathbb{R}^{4}, 0\right) \mid F(x, y, \lambda)=\frac{\partial F}{\partial x}(x, y, \lambda)=\frac{\partial F}{\partial y}(x, y, \lambda)=0\right\} .
$$

The normal forms are given by

$$
\begin{aligned}
& A_{k} ; \quad F(x, y, \lambda)=x^{k+1} \pm y^{2}+\lambda_{1}+\lambda_{2} x+\cdots+\lambda_{k-1} x^{k-1}, 1 \leq k \leq 4, \\
& D_{4}^{+} ; \quad F(x, y, \lambda)=x^{3}+y^{3}+\lambda_{1}+\lambda_{2} x+\lambda_{3} y+\lambda_{4} x y, \\
& D_{4}^{-} ; \quad F(x, y, \lambda)=\frac{x^{3}}{3}-x y^{2}+\lambda_{1}+\lambda_{2} x+\lambda_{3} y+\lambda_{4}\left(x^{2}+y^{2}\right) .
\end{aligned}
$$

For example, if we consider the germ given by

$$
F(x, y, \lambda)=x^{3}+y^{3}+\lambda_{1}+\lambda_{2} x+\lambda_{3} y+\lambda_{4} x y .
$$

Then, we get

$$
\Sigma_{*}(F)=\left\{\left(x, y, 2\left(x^{3}+y^{3}\right)+\lambda_{4} x y,-3 x^{2}-\lambda_{4} y,-3 y^{2}-\lambda_{4} x, \lambda_{4}\right) \mid\left(x, y, \lambda_{4}\right) \in \mathbb{R}^{3}\right\} .
$$

Therefore, the corresponding Legendrian map germ is

$$
f\left(u_{1}, u_{2}, u_{3}\right)=\left(2\left(u_{1}^{2}+u_{2}^{2}\right)+u_{1} u_{2} u_{3}, 3 u_{1}^{2}+u_{2} u_{3}, 3 u_{2}^{2}+u_{1} u_{3}, u_{3}\right) \quad\left(D_{4}^{+}\right) .
$$

The other cases follow from similar arguments, so that we omit the details.

As a corollary of the above theorem, we have the following generic local classification of AdSlightlike evolutes along spacelike surfaces. We define that $C(2,3,4)=\left\{\left(u_{1}^{2}, u_{1}^{3}, u_{1}^{4}\right) \mid u_{1} \in \mathbb{R}\right\}$ which is called a $(2,3,4)$-cusp. We also define that $C(B F)=\left\{\left(10 u_{1}^{3}+3 u_{2} u_{1}, 5 u_{1}^{4}+u_{2} u_{1}^{2}, 6 u_{1}^{5}+u_{2} u_{1}^{3}, u_{2}\right) \mid\left(u_{1}\right.\right.$, $\left.\left.u_{2}\right) \in \mathbb{R}^{2}\right\}$. We call $C(B F)$ a $C$-butterfly (i.e., the critical value set of the butterfly). Finally, we define that where $C(2,3,4,5)=\left\{\left(u_{1}^{2}, u_{1}^{3}, u_{1}^{4}, u_{1}^{5}\right) \mid u_{1} \in \mathbb{R}\right\}$ which is called a $(2,3,4,5)$-cusp.

Corollary 6.5. There exists an open dense subset $O \subset \operatorname{Emb}_{\mathrm{sp}}\left(U, A d S^{4}\right)$ such that for any $\boldsymbol{X} \in$ $O$, the germ of the corresponding AdS-lightlike evolute $\mathbb{L}_{\mathbb{E}_{M}^{ \pm}}$at any point $\left(u_{0}, \mu_{0}\right) \in U \times \mathbb{R}$ is diffeomorphic to one of the following set germs at the origin in $\mathbb{R}^{4}$ :

$A_{2}:\{(0,0)\} \times \mathbb{R}^{2}$,

$A_{3}: C(2,3,4) \times \mathbb{R}$

$A_{4}: C(B F)$,

$D_{4}^{+}:\left\{\left(2\left(u_{1}^{3}+u_{2}^{3}\right)+u_{1} u_{2} u_{3}, 3 u_{1}^{2}+u_{2} u_{3}, 3 u_{2}^{2}+u_{1} u_{3}, u_{3}\right) \mid u_{3}^{2}=36 u_{1} u_{2}\right\}$,

$D_{4}^{-} ;\left\{\left(\left(\frac{u_{1}^{3}}{3}-u_{1} u_{2}^{2}\right)+\left(u_{1}^{2}+u_{2}^{2}\right) u_{3}, u_{2}^{2}-u_{1}^{2}-2 u_{1} u_{3}, 2\left(u_{1} u_{2}-u_{2} u_{3}\right), u_{3}\right) \mid u_{3}^{2}=u_{1}^{2}+u_{2}^{2}\right\}$.

Proof. For $A_{3}$, we can calculate the Jacobi matrix of the normal form $f$ in Theorem 6.4,

$$
J_{f}=\left(\begin{array}{ccc}
12 u_{1}^{2}+2 u_{1} & 2 u_{1} & 0 \\
12 u_{1}^{3}+2 u_{1} u_{2} & u_{1}^{2} & 0 \\
0 & 1 & 0 \\
0 & 0 & 1
\end{array}\right),
$$

so that rank $J_{f}<3$ if and only if $6 u_{1}^{2}+u_{2}=0$. Thus, the critical value set of $f$ is

$$
C(f)=\left\{\left(-8 u_{1}^{3},-3 u_{1}^{4},-6 u_{1}^{2}, u_{3}\right) \mid\left(u_{1}, u_{3}\right) \in \mathbb{R}^{2}\right\} .
$$

It is $C(2,3,4) \times \mathbb{R}$. By a similar calculation, we can show that the germ of $A_{4}$ is diffeomorphic to $C(B F)$. For $D_{4}^{+}$, we can calculate the Jacobi matrix o the normal form $f$,

$$
J_{f}=\left(\begin{array}{ccc}
6 u_{1}^{2}+u_{2} u_{3} & 6 u_{2}^{2}+u_{1} u_{3}, u_{1} u_{2} & 0 \\
6 u_{1} & u_{3} & u_{2} \\
u_{3} & 6 u_{2} & u_{1} \\
0 & 0 & 1
\end{array}\right) .
$$


Therefore, rank $J_{f}<3$ if and only if

$$
\left|\begin{array}{cc}
6 u_{1}^{2}+u_{2} u_{3} & 6 u_{2}^{2}+u_{1} u_{3}, u_{1} u_{2} \\
6 u_{1} & u_{3}
\end{array}\right|=\left|\begin{array}{cc}
6 u_{1}^{2}+u_{2} u_{3} & 6 u_{2}^{2}+u_{1} u_{3}, u_{1} u_{2} \\
u_{3} & 6 u_{2}
\end{array}\right|=\left|\begin{array}{cc}
6 u_{1} & u_{3} \\
u_{3} & 6 u_{2}
\end{array}\right|=0,
$$

which is equivalent to the condition that $u_{3}^{2}=36 u_{1} u_{2}$. For $D_{4}^{-}$, by a similar calculation to the above, we have the condition that $u_{3}^{2}=u_{1}^{2}+u_{2}^{2}$. This completes the proof.

In the list of the above corollary, one of the projections of the image of $D_{4}^{+}$into $\mathbb{R}^{3}$ is $P U=\left\{\left(3 u_{1}^{2}+u_{2} u_{3}, 3 u_{2}^{2}+u_{1} u_{3}, u_{3}\right) \mid u_{3}^{2}=36 u_{1} u_{2}\right\}$ which is called a purse and one of the projections of the image of $D_{4}^{-}$is called a pyramid given by

$$
P Y=\left\{\left(u_{2}^{2}-u_{1}^{2}-2 u_{1} u_{3}, 2\left(u_{1} u_{2}-u_{2} u_{3}\right), u_{3}\right) \mid u_{3}^{2}=u_{1}^{2}+u_{2}^{2}\right\} .
$$

We can draw these pictures as follows. We denote $\widetilde{P U}$ as the set of $D_{4}^{+}$and $\widetilde{P Y}$ as the set of $D_{4}^{-}$in the list of Corollary 6.5, respectively. We also have a classification of the singularities of $\mathbb{L} \mathbb{E}_{M}^{ \pm}$as a corollary of Theorem 6.4 and Corollary 6.5. The set of singularities of $\mathbb{L} \mathbb{E}_{M}^{ \pm}$is denoted by $\Sigma\left(\mathbb{L} \mathbb{E}_{M}^{ \pm}\right)$.

Corollary 6.6. There exists an open dense subset $O \subset \operatorname{Emb}_{\mathrm{sp}}\left(U, A d S^{4}\right)$ such that for any $\boldsymbol{X} \in$ $O$, the germ of the pair $\left(\mathbb{L E}_{M}^{ \pm}, \Sigma\left(\mathbb{L} \mathbb{E}_{M}^{ \pm}\right)\right)$at any point $\lambda_{0} \in \Sigma\left(\mathbb{L} \mathbb{E}_{M}^{ \pm}\right)$is diffeomorphic to one of the following pairs of set germs at the origin in $\mathbb{R}^{4}$ :

$A_{3}:(C(2,3,4) \times \mathbb{R},\{(0,0,0)\} \times \mathbb{R})$,

$A_{4}:(C(B F), C(2,3,4,5))$,

$D_{4}^{+}:(\widetilde{P U}, \Sigma(\widetilde{P U}))$, where $\Sigma(\widetilde{P U})=\left\{\left(5 u_{3}^{3} / 108, u_{3}^{2} / 4, u_{3}^{2} / 4, u_{3}\right) \mid u_{3} \in \mathbb{R}\right\}$,

$D_{4}^{-}:(\widetilde{P Y}, \Sigma(\widetilde{P Y}))$, where

$\Sigma(\widetilde{P Y}))=\left\{\left(4 u_{3}^{3} / 3,-3 u_{3}^{2}, 0, u_{3}\right) \mid u_{3} \in \mathbb{R}\right\} \cup$

$\left\{\left(4 u_{3}^{3} / 3,3 u_{3}^{2} / 2,-3 \sqrt{3} u_{3}^{2} / 2, u_{3}\right) \mid u_{3} \in \mathbb{R}\right\} \cup\left\{\left(4 u_{3}^{3} / 3,3 u_{3}^{2} / 2,3 \sqrt{3} u_{3}^{2} / 2, u_{3}\right) \mid u_{3} \in \mathbb{R}\right\}$.

Proof. For $A_{3}$, in order to detect the singularities, we calculate the Jacobi matrix of $f\left(u_{1}, u_{2}\right)=$ $\left(u_{1}^{2}, u_{1}^{3}, u_{1}^{4}, u_{2}\right)$. Then, we have

$$
J_{f}=\left(\begin{array}{cc}
2 u_{1} & 0 \\
3 u_{1}^{2} & 0 \\
4 u_{1}^{3} & 0 \\
0 & 1
\end{array}\right),
$$

so that rank $J_{f}<2$ if and only if $u_{1}=0$. This means that $\Sigma(C(2,3,4) \times \mathbb{R})=\{(0,0,0)\} \times \mathbb{R}$. Similar to the case $A_{3}$, we calculate the Jacobi matrix of $f\left(u_{1}, u_{2}\right)=\left(10 u_{1}^{3}+3 u_{2} u_{1}, 5 u_{1}^{4}+u_{2} u_{1}^{2}, 6 u_{1}^{5}+u_{2} u_{1}^{3}, u_{2}\right)$. Then, we have

$$
J_{f}=\left(\begin{array}{cc}
30 u_{1}^{2}+3 u_{2} & 3 u_{1} \\
20 u_{1}^{3}+2 u_{2} u_{2} & u_{1}^{2} \\
30 u_{1}^{4}+3 u_{2} u_{1}^{2} & u_{1}^{3} \\
0 & 1
\end{array}\right),
$$

so that rank $J_{f}<2$ if and only if $u_{2}=-10 u_{1}^{2}$. Therefore, we have

$$
f\left(u_{1},-10 u_{1}^{2}\right)=\left(-20 u_{1}^{3},-5 u_{1}^{4},-4 u_{1}^{5},-10 u_{1}^{2}\right) .
$$

This means that $\Sigma(C(B F))=C(2,3,4,5)$. For $D_{4}^{+}$, we consider the following parameter transformation:

$$
u_{1}+u_{2}=\frac{u_{3}}{3} \cosh \phi, u_{1}-u_{2}=\frac{u_{3}}{3} \sinh \phi .
$$

Then, $\widetilde{P U}$ is parametrized by

$$
f\left(\phi, u_{3}\right)=\left(\frac{4}{6^{3}} u_{3}^{3}\left(\cosh 3 \phi+\frac{1}{4}\right), \frac{u_{3}^{2}}{6^{2}}\left(3 e^{2 \phi}+6 e^{-\phi}\right), \frac{u_{3}^{2}}{6^{2}}\left(3 e^{-2 \phi}+e^{\phi}\right), u_{3}\right) .
$$


Thus, the Jacobi matrix is

$$
J_{f}=\left(\begin{array}{cc}
\frac{1}{18} u_{3}^{3} \sinh 3 \phi & \frac{1}{18} u_{3}^{2}\left(\cosh 3 \phi+\frac{1}{4}\right) \\
\frac{u_{3}^{2}}{6}\left(e^{2 \phi}-e^{-\phi}\right) & \frac{1}{6}\left(e^{2 \phi}+2 e^{-\phi}\right) \\
\frac{u_{3}^{2}}{6}\left(-e^{-2 \phi}+e^{\phi}\right) & \frac{1}{6}\left(e^{-2 \phi}+2 e^{\phi}\right) \\
0 & 1
\end{array}\right),
$$

so that rank $J_{f}<2$ if and only if $\phi=0$ or $u_{3}=0$. This means that $u_{1}=u_{3} / 6, u_{2}=u_{3} / 6$. Therefore, we have

$$
\Sigma(\widetilde{P U})=\left\{\left(\frac{5}{108} u_{3}^{3}, \frac{1}{4} u_{3}^{2}, \frac{1}{4} u_{3}^{2}, u_{3}\right) \mid u_{3} \in \mathbb{R}\right\} .
$$

For $D_{4}^{-}$, we also consider the following parameter transformation:

$$
u_{1}=u_{3} \cos \theta, u_{2}=u_{3} \sin \theta \text {. }
$$

Then, $\widetilde{P Y}$ is parametrized by

$$
f\left(\theta, u_{3}\right)=\left(\frac{1}{3} u_{3}^{3}(\cos 3 \theta+1),-u_{3}^{2}(\cos 2 \theta+2 \cos \theta), 2 u_{3}^{2}(\sin 2 \theta-2 \sin \theta), u_{3}\right) .
$$

Thus, the Jacobi matrix is

$$
J_{f}=\left(\begin{array}{cc}
-u_{3}^{3} \sin 3 \theta & u_{3}^{2}(\cos 3 \theta+1) \\
2 u_{3}^{2}(\sin 2 \theta+\sin \theta) & -2 u_{3}(\cos 2 \theta+2 \cos \theta) \\
2 u_{3}^{2}(\cos 2 \theta-\cos \theta) & 2 u_{3}(\sin 2 \theta-2 \sin \theta) \\
0 & 1
\end{array}\right),
$$

so that $\operatorname{rank} J_{f}<2$ if and only if $\theta=0,2 \pi / 3,5 \pi / 3$ or $u_{3}=0$. If $\theta=0$, then we have $u_{1}=u_{3}, u_{2}=0$, so that we have $\left\{\left(4 u_{3}^{3} / 3,-3 u_{3}^{2}, 0, u_{3}\right) \mid u_{3} \in \mathbb{R}\right\}$. If $\theta=2 \pi / 3$, then we have $u_{1}=-u_{3} / 2, u_{2}=\sqrt{3} u_{3} / 2$, so that we have $\left\{\left(4 u_{3}^{3} / 3,3 u_{3}^{2} / 2,-3 \sqrt{3} u_{3}^{2} / 2, u_{3} \mid u_{3} \in \mathbb{R}\right\}\right.$. Finally, if $\theta=5 \pi / 3$, then we have $u=$ $-u_{3} / 2, u_{2}=-\sqrt{3} u_{3} / 2$, so that we have $\left\{\left(4 u_{3}^{3} / 3,3 u_{3}^{2} / 2,3 \sqrt{3} u_{3}^{2} / 2, u_{3}\right) \mid u_{3} \in \mathbb{R}\right\}$. It follows that $\left.\Sigma(\widetilde{P Y})\right)$ is the union of these three set germs. This completes the proof.

We can interpret the geometric meaning of the above normal forms of the singularities of lightlike hypersurfaces as the following theorem shows.

Theorem 6.7. Let $O \subset \operatorname{Emb}_{\mathrm{sp}}\left(U, A d S^{4}\right)$ be the open dense subset given in the above theorem. For any $\boldsymbol{X} \in O$, the germ of the corresponding lightlike hypersurfaces $\left(\mathbb{L} \mathbb{H}_{M}^{ \pm}, \lambda_{0}\right)$ at a point $\left(u_{0}, \mu_{0}\right) \in U \times \mathbb{R}$ is characterized as follows :

(1) $\quad\left(A_{1}\right)$ if and only if $\lambda_{0} \in \mathbb{L} \mathbb{H}_{M}^{+}(U \times \mathbb{R}) \cup \mathbb{L} \mathbb{H}_{M}^{-}(U \times \mathbb{R})$ is a non-singular point.

(2) $\quad\left(A_{2}\right)$ if and only if $p_{0}=X\left(u_{0}\right)$ is not an $A d S$-lightlike ridge point and $\lambda_{0} \in \mathbb{L E}_{M}^{+} \cup \mathbb{L} \mathbb{E}_{M}^{-}$.

(3) $\left(A_{3}\right)$ if and only if $p_{0}=\boldsymbol{X}\left(u_{0}\right)$ is an AdS-lightlike 1-ridge point and $\lambda_{0} \in \mathbb{L}_{M}^{+} \cup \mathbb{L} \mathbb{E}_{M}^{-}$.

(4) $\left(A_{4}\right)$ if and only if $p_{0}=X\left(u_{0}\right)$ is an AdS-lightlike 2-ridge point, $\lambda_{0} \in \mathbb{L}_{M}^{+} \cup \mathbb{L} \mathbb{E}_{M}^{-}$and $u_{0} \in$ $U$ satisfies the following condition: For sufficiently small $\varepsilon>0$, there exist two different AdS-lightlike 1-ridge points $u^{1}, u^{2} \in U$ such that $\left|u_{0}-u^{i}\right|<\varepsilon(i=1,2)$.

(5) ( $\left.D_{4}^{+}\right)$if and only if $\lambda_{0} \in \mathbb{L E}_{M}^{+} \cup \mathbb{L} \mathbb{E}_{M}^{-}, p_{0}=X\left(u_{0}\right)$ has corank two contact with the osculating lightcone and $u_{0} \in U$ satisfies the following condition :

(a) $\kappa_{1}\left(\boldsymbol{n}^{T}, \pm \boldsymbol{n}^{S}\right)\left(u^{0}\right)=\kappa_{2}\left(\boldsymbol{n}^{T}, \pm \boldsymbol{n}^{S}\right)\left(u^{0}\right)$.

(b) For sufficiently small $\varepsilon>0$, there exist two different AdS-lightlike 1-ridge points $u^{1}, u^{2}$ $\in U$ such that $\left|u_{0}-u^{i}\right|<\varepsilon,(i=1,2)$ and $\kappa_{1}\left(\boldsymbol{n}^{T}, \pm \boldsymbol{n}^{S}\right)\left(u^{i}\right) \neq \kappa_{2}\left(\boldsymbol{n}^{T}, \pm \boldsymbol{n}^{S}\right)\left(u^{i}\right)$.

(6) $\left(D_{4}^{-}\right)$if and only if $\lambda_{0} \in \mathbb{L}_{M}, p_{0}=X\left(u_{0}\right)$ has corank two contact with the osculating lightcone and $u_{0} \in U$ satisfies the following condition:

(a) $\quad \kappa_{1}\left(\boldsymbol{n}^{T}, \pm \boldsymbol{n}^{S}\right)\left(u^{0}\right)=\kappa_{2}\left(\boldsymbol{n}^{T}, \pm \boldsymbol{n}^{S}\right)\left(u^{0}\right)$. 
(b) For sufficiently small $\varepsilon>0$, there exist three different AdS-lightlike 1-ridge points $u^{i} \in U,(i=1,2,3)$ such that $\left|u_{0}-u^{i}\right|<\varepsilon$ and $\kappa_{1}\left(\boldsymbol{n}^{T}, \pm \boldsymbol{n}^{S}\right)\left(u^{i}\right) \neq \kappa_{2}\left(\boldsymbol{n}^{T}, \pm \boldsymbol{n}^{S}\right)\left(u^{i}\right)$.

Proof. By normal forms $\left(A_{1}\right)$ and $\left(A_{2}\right)$ in Theorem 6.4, assertions (1) and (2) are trivial. For the normal form $\left(A_{3}\right)$, the AdS-height function has the $A_{3}$ singularity at $p_{0}=\boldsymbol{X}\left(u_{0}\right)$, so that it is an AdS-lightlike 1-ridge point.

Here, we give a remark on the classification of $\mathcal{K}$-simple singularities of function germs. In the list of the classification, we say that a class of singularities $L$ is adjacent to a class $K$ (notation: $K \leftarrow L$ ) if every function germ $f \in L$ can be deformed into a function of $K$ by an arbitrarily small perturbation. For the class of $A_{k}, D_{k}^{ \pm}$of the $\mathcal{K}$-classification are adjacent to each other as follows (Ref. 1, p. 243):

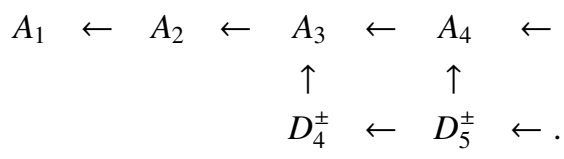

By the normal form $\left(A_{4}\right)$, the singularities of the AdS-lightcone evolute are a $(2,3,4,5)$-cusp. Therefore, two singular loci approach to the $(2,3,4,5)$-cusp point. Since $A_{4}$ is adjacent to $A_{3}$, the singular loci consist of AdS-lightlike 1-ridge points except the origin. Thus, assertion (4) holds. For $\left(D_{4}^{+}\right)$, the singularities of the AdS-lightcone evolute are $\Sigma(\widetilde{P U})$. By the normal form of the generating family, the corank of the AdS-height function at $u^{0} \in U$ is two, so that it is an $\left(\boldsymbol{n}^{T}\left(u_{0}\right), \pm \boldsymbol{n}^{S}\left(u_{0}\right)\right)$-umbilical point. Therefore, we have $\kappa_{1}\left(\boldsymbol{n}^{T}, \pm \boldsymbol{n}^{S}\right)\left(u^{0}\right)=\kappa_{2}\left(\boldsymbol{n}^{T}, \pm \boldsymbol{n}^{S}\right)\left(u^{0}\right)$. By the normal form of $\left(D_{4}^{+}\right)$in Corollary 6.6, two singular loci approach to the origin from both the positive and the negative sides of the parameter $u_{3}$. Since $D_{4}^{+}$is adjacent to $A_{3}$, the singular loci consist of AdS-lightlike 1-ridge points except at the origin. Of course, an AdS-lightlike 1-ridge point is not an $\left(\boldsymbol{n}^{T}, \pm \boldsymbol{n}^{S}\right)$-umbilical point. So that two nullcone principal curvatures are different at an AdS-lightlike 1-ridge point. For $\left(D_{4}^{-}\right)$, we have the assertion by the similar arguments to the case $\left(D_{4}^{+}\right)$. This completes the proof.

\section{SPACELIKE CURVES IN $A d S^{4}$}

In this section, we consider lightlike hypersurfaces along spacelike curves in $A d S^{4}$ as the simplest case of higher codimension. Let $\gamma: I \longrightarrow A d S^{4}$ be a spacelike curve with $\left\|\gamma^{\prime \prime}(s)\right\| \neq-1$. In this case, we write $C=\gamma(I)$ instead of $M=\gamma(I)$. Since $\left\|\gamma^{\prime}(s)\right\|>0$, we can reparameterize it by the arc-length s. So, we have the unit tangent vector $\boldsymbol{t}(s)=\boldsymbol{\gamma}^{\prime}(s)$ of $\boldsymbol{\gamma}(s)$. Moreover, we have two unit normal vectors $\boldsymbol{n}_{1}(s)=\frac{\boldsymbol{\gamma}^{\prime \prime}(s)-\boldsymbol{\gamma}(s)}{\left\|\boldsymbol{\gamma}^{\prime \prime}(s)-\boldsymbol{\gamma}(s)\right\|}, \boldsymbol{n}_{2}(s)=\frac{\boldsymbol{n}_{1}^{\prime}(s)+\delta \kappa_{1}(s) \boldsymbol{t}(s)}{\left\|\boldsymbol{n}_{1}^{\prime}(s)+\delta \kappa_{1}(s) \boldsymbol{t}(s)\right\|}$ under the conditions that $\kappa_{1}(s)=\left\|\boldsymbol{\gamma}^{\prime \prime}(s)-\boldsymbol{\gamma}(s)\right\| \neq 0, \kappa_{2}(s)=\left\|\boldsymbol{n}_{1}^{\prime}(s)+\delta k_{1}(s) \boldsymbol{t}(s)\right\| \neq 0$, where $\delta_{i}=\operatorname{sign}\left(\boldsymbol{n}_{i}(s)\right)$ and $\operatorname{sign}\left(\boldsymbol{n}_{i}(s)\right)$ is the signature of $\boldsymbol{n}_{i}(s)(i=1,2,3)$. Then, we have another unit normal vector field $\boldsymbol{n}_{3}(s)$ defined by $\boldsymbol{n}_{3}(s)=\boldsymbol{\gamma}(s) \wedge \boldsymbol{t}(s) \wedge \boldsymbol{n}_{1}(s) \wedge \boldsymbol{n}_{2}(s)$. Therefore, we can construct a pseudo-orthogonal frame $\left\{\boldsymbol{\gamma}(s), \boldsymbol{t}(s), \boldsymbol{n}_{1}(s), \boldsymbol{n}_{2}(s), \boldsymbol{n}_{3}(s)\right\}$, which satisfies the Frenet-Serret type formulae,

$$
\left\{\begin{array}{l}
\boldsymbol{\gamma}^{\prime}(s)=\boldsymbol{t}(s), \\
\boldsymbol{t}^{\prime}(s)=\gamma(s)+\kappa_{1}(s) \boldsymbol{n}_{1}(s), \\
\boldsymbol{n}_{1}^{\prime}(s)=-\delta_{1} \kappa_{1}(s) \boldsymbol{t}(s)+\kappa_{2}(s) \boldsymbol{n}_{2}(s), \\
\boldsymbol{n}_{2}^{\prime}(s)=\delta_{3} \kappa_{2}(s) \boldsymbol{n}_{1}(s)+\kappa_{3}(s) \boldsymbol{n}_{3}(s), \\
\boldsymbol{n}_{3}^{\prime}(s)=\delta_{1} \kappa_{3}(s) n_{2}(s),
\end{array}\right.
$$

where $\kappa_{2}(s)=\delta_{2}\left\langle\boldsymbol{n}_{1}^{\prime}(s), \boldsymbol{n}_{2}(s)\right\rangle$ and $\kappa_{3}(s)=\delta_{3}\left\langle\boldsymbol{n}_{2}^{\prime}(s), \boldsymbol{n}_{3}(s)\right\rangle$. Since $\boldsymbol{\gamma}(s)$ is timelike and $\boldsymbol{t}(s)$ is spacelike, we distinguish the following three cases:

Case1: $\boldsymbol{n}_{1}(s)$ is timelike, that is, $\delta_{1}=-1$ and $\delta_{2}=\delta_{3}=1$.

Case2: $\boldsymbol{n}_{2}(s)$ is timelike, that is, $\delta_{2}=-1$ and $\delta_{1}=\delta_{3}=1$.

Case3: $\boldsymbol{n}_{3}(s)$ is timelike, that is, $\delta_{3}=-1$ and $\delta_{1}=\delta_{2}=1$. 
We consider the lightlike hypersurface along $C$ and calculate the anti-de Sitter height function on $C$ which is useful for the study the singularities of lightlike hypersurfaces in the each case.

\section{A. Case 1}

Suppose that $\boldsymbol{n}_{1}(s)$ is timelike. In this case we adopt $\boldsymbol{n}^{T}(s)=\boldsymbol{n}_{1}(s)$ and denote that $\boldsymbol{b}_{1}(s)=$ $\boldsymbol{n}_{2}(s), \boldsymbol{b}_{2}(s)=\boldsymbol{n}_{3}(s)$. Then, we have the pseudo-orthogonal frame

$$
\left\{\gamma(s), \boldsymbol{t}(s), \boldsymbol{n}^{T}(s), \boldsymbol{b}_{1}(s), \boldsymbol{b}_{2}(s)\right\},
$$

$\delta_{1}=-1$ and $\delta_{2}=\delta_{3}=1$, which satisfies the following Frenet-Serret type formulae:

$$
\left\{\begin{array}{l}
\gamma^{\prime}(s)=\boldsymbol{t}(s), \\
\boldsymbol{t}^{\prime}(s)=\gamma(s)+\kappa_{1}(s) \boldsymbol{n}^{T}(s), \\
\boldsymbol{n}^{T^{\prime}}(s)=\kappa_{1}(s) \boldsymbol{t}(s)+\kappa_{2}(s) \boldsymbol{b}_{1}(s), \\
\boldsymbol{b}_{1}^{\prime}(s)=\kappa_{2}(s) \boldsymbol{n}^{T}(s)+\kappa_{3}(s) \boldsymbol{b}_{2}(s), \\
\boldsymbol{b}_{2}^{\prime}(s)=-\kappa_{3}(s) \boldsymbol{b}_{1}(s) .
\end{array}\right.
$$

Since $N_{1}(C)\left[\boldsymbol{n}^{T}\right]$ is parametrized by

$$
N_{1}(C)\left[\boldsymbol{n}^{T}\right]=\left\{(\boldsymbol{\gamma}(s), \boldsymbol{\xi}) \in \boldsymbol{\gamma}^{*} T \mathbb{R}_{1}^{5} \mid \boldsymbol{\xi}=\cos \theta \boldsymbol{b}_{1}(s)+\sin \theta \boldsymbol{b}_{2}(s) \in N_{\boldsymbol{\gamma}(s)}(C), s \in I\right\},
$$

the nullcone Gauss image of $N_{1}(C)_{p}\left[\boldsymbol{n}^{T}\right]$ is given by

$$
\mathbb{N G}\left(\boldsymbol{n}^{T}\right)(s, \theta)=\boldsymbol{n}^{T}(s)+\cos \theta \boldsymbol{b}_{1}(s)+\sin \theta \boldsymbol{b}_{2}(s) .
$$

Then, we have the lightlike hypersurface along $C$,

$$
\mathbb{L} \mathbb{H}_{C}((s, \theta), \mu)=\gamma(s)+\mu\left(\boldsymbol{n}^{T}(s)+\cos \theta \boldsymbol{b}_{1}(s)+\sin \theta \boldsymbol{b}_{2}(s)\right)=\gamma(s)+\mu \mathbb{N} G\left(n^{T}\right)(s, \theta) .
$$

We remark that the image of this lightlike hypersurface along $C$ is independent of the choice of the future directed timelike normal vector field $\boldsymbol{n}^{T}$ by Corollary 4.3.

Now we investigate the anti-de Sitter height functions $H: I \times A d S^{4} \longrightarrow \mathbb{R}$ on a spacelike curve $C=\gamma(I)$ defined by

$$
H(p, \lambda)=H(s, \lambda)=\langle\gamma(s), \lambda\rangle+1,
$$

where $p=\gamma(s)$. For any fixed $\lambda_{0} \in A d S^{4}$, we write $h(p)=H_{\lambda_{0}}(p)=H\left(p, \lambda_{0}\right)$.

By Proposition 4.1, the discriminant set of the anti-de Sitter height function $H$ is given by

$$
\mathcal{D}_{H}=\mathbb{L H}_{C}\left(N_{1}(C)\left[\boldsymbol{n}^{T}\right] \times \mathbb{R}\right)=\{\lambda=\gamma(s)+\mu \mathbb{N} G(s, \theta) \mid \theta \in[0,2 \pi), s \in I, \mu \in \mathbb{R}\},
$$

which is the image of the lightlike hypersurface along $C$. We also calculate that $h^{\prime \prime}(p)=\left\langle\gamma^{\prime \prime}(s), \lambda_{0}\right\rangle$ $=-\mu \kappa_{1}-1$. Then, $h^{\prime \prime}(p)=0$ if and only if $\mu=-1 / \kappa_{1}(s)$. It means that a singular point of the lightlike hypersurface is a point $\lambda_{0}=\gamma\left(s_{0}\right)+\mu_{0} \mathbb{N} G\left(\theta_{0}, s_{0}\right)$ for $\mu_{0}=-1 / \kappa_{1}\left(s_{0}\right)$. Therefore, the lightlike focal surface is

$$
\mathbb{L F}_{C}=\left\{\lambda=\gamma(s)-\frac{1}{\kappa_{1}(s)} \mathbb{N G}\left(\boldsymbol{n}^{T}\right)(s, \theta) \mid s \in I, \theta \in[0,2 \pi)\right\} .
$$

Moreover, if we calculate the 3rd, 4th, and 5th derivatives of $h(s)$, we have the following proposition.

Proposition 7.1. Let $C$ be a spacelike curve and $H: C \times\left(A d S^{4} \backslash C\right) \rightarrow \mathbb{R}$ the anti-de Sitter height function on $C$. Suppose that $p_{0}=\gamma\left(s_{0}\right) \neq \lambda_{0}$. Then, we have the following:

$$
h\left(p_{0}\right)=h^{\prime}\left(p_{0}\right)=0 \text { if and only if there exist } \theta_{0} \in[0,2 \pi) \text { and } \mu \in \mathbb{R} \backslash\{0\} \text { such that }
$$

$$
\gamma\left(s_{0}\right)-\lambda_{0}=\mu \mathbb{N} G\left(\boldsymbol{n}^{T}\right)\left(s_{0}, \theta_{0}\right) .
$$

(2) $\quad h\left(p_{0}\right)=h^{\prime}\left(p_{0}\right)=h^{\prime \prime}\left(p_{0}\right)=0$ if and only if there exists $\theta_{0} \in[0,2 \pi)$ such that

$$
\gamma\left(s_{0}\right)-\lambda_{0}=-\frac{1}{\kappa_{1}\left(s_{0}\right)} \mathbb{N} \mathbb{G}\left(\boldsymbol{n}^{T}\right)\left(s_{0}, \theta_{0}\right) .
$$


(3) $\quad h\left(p_{0}\right)=h^{\prime}\left(p_{0}\right)=h^{\prime \prime}\left(p_{0}\right)=h^{\prime \prime \prime}\left(p_{0}\right)=0$ if and only if there exists $\theta_{0} \in[0,2 \pi)$ such that

$$
\gamma\left(s_{0}\right)-\lambda_{0}=-\frac{1}{\kappa_{1}\left(s_{0}\right)} \mathbb{N} \mathbb{G}\left(\boldsymbol{n}^{T}\right)\left(s_{0}, \theta_{0}\right)
$$

and $\kappa_{1}^{\prime}\left(s_{0}\right)-\cos \theta_{0} \kappa_{1}\left(s_{0}\right) \kappa_{2}\left(s_{0}\right)=0$, so that we can write $\theta_{0}=\theta\left(s_{0}\right)$.

(4) $h\left(p_{0}\right)=h^{\prime}\left(p_{0}\right)=h^{\prime \prime}\left(p_{0}\right)=h^{\prime \prime \prime}\left(p_{0}\right)=h^{(4)}\left(p_{0}\right)=0$ if and only if there exists $\theta_{0}=\theta\left(s_{0}\right) \in[0,2 \pi)$ such that

$$
\gamma\left(s_{0}\right)-\lambda_{0}=-\frac{1}{\kappa_{1}\left(s_{0}\right)} \mathbb{N} \mathbb{G}\left(\boldsymbol{n}^{T}\right)\left(s_{0}, \theta\left(s_{0}\right)\right),
$$

$\kappa_{1}^{\prime}\left(s_{0}\right)-\cos \theta\left(s_{0}\right) \kappa_{1}\left(s_{0}\right) \kappa_{2}\left(s_{0}\right)=0 \quad$ and $\quad\left(2 \kappa_{1}^{\prime}\left(s_{0}\right) \kappa_{2}\left(s_{0}\right)+\kappa_{1}\left(s_{0}\right) \kappa_{2}^{\prime}\left(s_{0}\right)\right) \cos \theta\left(s_{0}\right)-\kappa_{1}^{\prime \prime}\left(s_{0}\right)-\kappa_{1}$ $\left(s_{0}\right) \kappa_{2}^{2}\left(s_{0}\right)+\kappa_{1}\left(s_{0}\right) \kappa_{2}\left(s_{0}\right) \kappa_{3}\left(s_{0}\right) \sin \theta\left(s_{0}\right)=0$.

(5) $h\left(p_{0}\right)=h^{\prime}\left(p_{0}\right)=h^{\prime \prime}\left(p_{0}\right)=h^{\prime \prime \prime}\left(p_{0}\right)=h^{(4)}\left(p_{0}\right)=h^{(5)}\left(p_{0}\right)=0$ if and only if there exists $\theta_{0}=$ $\theta\left(s_{0}\right) \in[0,2 \pi)$ such that

$$
\begin{gathered}
\gamma\left(s_{0}\right)-\lambda_{0}=-\frac{1}{\kappa_{1}\left(s_{0}\right)} \mathbb{N} G\left(\boldsymbol{n}^{T}\right)\left(s_{0}, \theta\left(s_{0}\right)\right), \\
\kappa_{1}^{\prime}\left(s_{0}\right)-\cos \theta\left(s_{0}\right) \kappa_{1}\left(s_{0}\right) \kappa_{2}\left(s_{0}\right)=0, \quad\left(2 \kappa_{1}^{\prime}\left(s_{0}\right) \kappa_{2}\left(s_{0}\right)+\kappa_{1}\left(s_{0}\right) \kappa_{2}^{\prime}\left(s_{0}\right)\right) \cos \theta\left(s_{0}\right)-\kappa_{1}^{\prime \prime}\left(s_{0}\right)-\kappa_{1}\left(s_{0}\right) \\
\kappa_{2}^{2}\left(s_{0}\right)+\kappa_{1}\left(s_{0}\right) \kappa_{2}\left(s_{0}\right) \kappa_{3}\left(s_{0}\right) \sin \theta\left(s_{0}\right)=0 \quad 0 \quad \text { and }\left(\left(2 \kappa_{1}^{\prime}\left(s_{0}\right) \kappa_{2}\left(s_{0}\right)+\kappa_{1}\left(s_{0}\right) \kappa_{2}^{\prime}\left(s_{0}\right)\right) \cos \theta\left(s_{0}\right)\right. \\
\left.-\kappa_{1}^{\prime \prime}\left(s_{0}\right)-\kappa_{1}\left(s_{0}\right) \kappa_{2}^{2}\left(s_{0}\right)+\kappa_{1}\left(s_{0}\right) \kappa_{2}\left(s_{0}\right) \kappa_{3}\left(s_{0}\right) \sin \theta\left(s_{0}\right)\right)^{\prime}=0 .
\end{gathered}
$$

Taking account of the above proposition, we denote that $\rho_{1}(s, \theta)=\kappa_{1}^{\prime}(s)-\cos \theta \kappa_{1}(s) \kappa_{2}(s)$ and $\eta_{1}(s, \theta)=\left(2 \kappa_{1}^{\prime}(s) \kappa_{2}(s)+\kappa_{1}(s) \kappa_{2}^{\prime}(s)\right) \cos \theta-\kappa_{1}^{\prime \prime}(s)-\kappa_{1}(s) \kappa_{2}^{2}(s)+\kappa_{1}(s) \kappa_{2}(s) \kappa_{3}(s) \sin \theta$, which might be important invariants of $C=\gamma(I)$. Then, we can show that $\rho_{1}(s, \theta)=\eta_{1}(s, \theta)=0$ if and only if $\rho_{1}(s, \theta)=\sigma_{1}(s)=0$, where

$$
\sigma_{1}(s)=\left[\kappa_{1} \kappa_{2}\left(\kappa_{1}^{\prime \prime}+\kappa_{1} \kappa_{2}^{2}\right)-\kappa_{1}^{\prime}\left(2 \kappa_{1}^{\prime} \kappa_{2}+\kappa_{1} \kappa_{2}^{\prime}\right) \mp \kappa_{1} \kappa_{2} \kappa_{3} \sqrt{\left(\kappa_{1} \kappa_{2}\right)^{2}-\left(\kappa_{1}^{\prime}\right)^{2}}\right](s) .
$$

\section{B. Case 2}

Suppose that $\boldsymbol{n}_{2}(s)$ is timelike. Then, we adopt $\boldsymbol{n}^{T}(s)=\boldsymbol{n}_{2}(s)$ and denote that $\boldsymbol{b}_{1}(s)=\boldsymbol{n}_{1}(s), \boldsymbol{b}_{2}(s)$ $=\boldsymbol{n}_{3}(s)$. We have a pseudo-orthogonal frame $\left\{\boldsymbol{\gamma}(s), \boldsymbol{t}(s), \boldsymbol{n}^{T}(s), \boldsymbol{b}_{1}(s), \boldsymbol{b}_{2}(s)\right\}, \delta_{2}=-1$ and $\delta_{1}=\delta_{3}=$ 1, which satisfies the following Frenet-Serret type formulae:

$$
\left\{\begin{array}{l}
\boldsymbol{\gamma}^{\prime}(s)=\boldsymbol{t}(s), \\
\boldsymbol{t}^{\prime}(s)=\gamma(s)+\kappa_{1}(s) \boldsymbol{b}_{1}(s), \\
\boldsymbol{b}_{1}^{\prime}(s)=-\kappa_{1}(s) \boldsymbol{t}(s)+\kappa_{2}(s) \boldsymbol{n}^{T}(s), \\
\boldsymbol{n}^{T^{\prime}}(s)=\kappa_{2}(s) \boldsymbol{b}_{1}(s)+\kappa_{3}(s) \boldsymbol{b}_{2}(s), \\
\boldsymbol{b}_{2}^{\prime}(s)=\kappa_{3}(s) \boldsymbol{n}^{T}(s),
\end{array}\right.
$$

Here, $N_{1}(C)\left[\boldsymbol{n}^{T}\right]$ is parametrized by

$$
N_{1}(C)\left[\boldsymbol{n}^{T}\right]=\left\{(\boldsymbol{\gamma}(s), \boldsymbol{\xi}) \in \boldsymbol{\gamma}^{*} T \mathbb{R}_{1}^{5} \mid \boldsymbol{\xi}=\cos \theta \boldsymbol{b}_{1}(s)+\sin \theta \boldsymbol{b}_{2}(s) \in N_{\boldsymbol{\gamma}(s)}(C), s \in I\right\},
$$

so that we have the lightlike hypersurface along $C=\gamma(I)$,

$$
\mathbb{L} \mathbb{H}_{C}((s, \theta), t)=\gamma(s)+\mu \mathbb{N} \mathbb{G}\left(\boldsymbol{n}^{T}\right)(s, \theta) .
$$

We consider the anti-de Sitter height function $H: I \times A d S^{4} \longrightarrow \mathbb{R}$ on a spacelike curve $C=$ $\gamma(I)$. Under the similar notations to case (1), we have the following proposition.

Proposition 7.2. Let $C$ be a spacelike curve and $H: C \times\left(A d S^{4} \backslash C\right) \rightarrow \mathbb{R}$ the anti-de Sitter height function on $C$. Suppose that $p_{0} \neq \lambda_{0}$. Then, we have the following:

(1) $h\left(p_{0}\right)=h^{\prime}\left(p_{0}\right)=0$ if and only if there exist $\theta_{0} \in[0,2 \pi)$ and $\mu \in \mathbb{R} \backslash\{0\}$ such that

$$
\gamma\left(s_{0}\right)-\lambda_{0}=\mu \mathbb{N} \mathbb{G}\left(\boldsymbol{n}^{T}\right)\left(s_{0}, \theta_{0}\right) .
$$


(2) $\quad h\left(p_{0}\right)=h^{\prime}\left(p_{0}\right)=h^{\prime \prime}\left(p_{0}\right)=0$ if and only if there exists $\theta_{0} \in[0,2 \pi)$ such that

$$
\boldsymbol{\gamma}\left(s_{0}\right)-\lambda_{0}=\frac{1}{\kappa_{1}\left(s_{0}\right) \cos \theta_{0}} \mathbb{N G}\left(\boldsymbol{n}^{T}\right)\left(s_{0}, \theta_{0}\right)
$$

(3) $h\left(p_{0}\right)=h^{\prime}\left(p_{0}\right)=h^{\prime \prime}\left(p_{0}\right)=h^{\prime \prime \prime}\left(p_{0}\right)=0$ if and only if there exists $\theta_{0} \in[0,2 \pi)$ such that

$$
\boldsymbol{\gamma}\left(s_{0}\right)-\lambda_{0}=\frac{1}{\kappa_{1}\left(s_{0}\right) \cos \theta_{0}} \mathbb{N} \mathbb{G}\left(\boldsymbol{n}^{T}\right)\left(s_{0}, \theta_{0}\right)
$$

and $\kappa_{1}^{\prime}\left(s_{0}\right) \cos \theta_{0}-\kappa_{1}\left(s_{0}\right) \kappa_{2}\left(s_{0}\right)=0$, so that we can write $\theta_{0}=\theta\left(s_{0}\right)$.

(4) $\quad h\left(p_{0}\right)=h^{\prime}\left(p_{0}\right)=h^{\prime \prime}\left(p_{0}\right)=h^{\prime \prime \prime}\left(p_{0}\right)=h^{(4)}\left(p_{0}\right)=0$ if and only if there exists $\theta_{0}=\theta\left(s_{0}\right) \in[0,2 \pi)$ such that

$$
\boldsymbol{\gamma}\left(s_{0}\right)-\lambda_{0}=\frac{1}{\kappa_{1}\left(s_{0}\right) \cos \theta\left(s_{0}\right)} \mathbb{N} G\left(\boldsymbol{n}^{T}\right)\left(s_{0}, \theta\left(s_{0}\right)\right),
$$

$\kappa_{1}^{\prime}\left(s_{0}\right) \cos \theta\left(s_{0}\right)-\kappa_{1}\left(s_{0}\right) \kappa_{2}\left(s_{0}\right)=0 \quad$ and $\quad\left(\kappa_{1}^{\prime \prime}\left(s_{0}\right)+\kappa_{1}\left(s_{0}\right) \kappa_{2}^{2}\left(s_{0}\right)\right) \cos \theta\left(s_{0}\right)-2 \kappa_{1}^{\prime}\left(s_{0}\right) \kappa_{2}\left(s_{0}\right)-\kappa_{1}$ $\left(s_{0}\right) \kappa_{2}^{\prime}\left(s_{0}\right)+\kappa_{1}\left(s_{0}\right) \kappa_{2}\left(s_{0}\right) \kappa_{3}\left(s_{0}\right) \sin \theta\left(s_{0}\right)=0$.

(5) $h\left(p_{0}\right)=h^{\prime}\left(p_{0}\right)=h^{\prime \prime}\left(p_{0}\right)=h^{\prime \prime \prime}\left(p_{0}\right)=h^{(4)}\left(p_{0}\right)=h^{(5)}\left(p_{0}\right)=0$ if and only if there exists $\theta_{0}=$ $\theta\left(s_{0}\right) \in[0,2 \pi)$ such that

$$
\boldsymbol{\gamma}\left(s_{0}\right)-\lambda_{0}=\frac{1}{\kappa_{1}\left(s_{0}\right) \cos \theta\left(s_{0}\right)} \mathbb{N} G\left(\boldsymbol{n}^{T}\right)\left(s_{0}, \theta\left(s_{0}\right)\right),
$$

$\kappa_{1}^{\prime}\left(s_{0}\right) \cos \theta\left(s_{0}\right)-\kappa_{1}\left(s_{0}\right) \kappa_{2}\left(s_{0}\right)=0, \quad\left(\kappa_{1}^{\prime \prime}\left(s_{0}\right)+\kappa_{1}\left(s_{0}\right) \kappa_{2}^{2}\left(s_{0}\right)\right) \cos \theta\left(s_{0}\right)-2 \kappa_{1}^{\prime}\left(s_{0}\right) \kappa_{2}\left(s_{0}\right)-\kappa_{1}\left(s_{0}\right)$ $\kappa_{2}^{\prime}\left(s_{0}\right)+\kappa_{1}\left(s_{0}\right) \kappa_{2}\left(s_{0}\right) \kappa_{3}\left(s_{0}\right) \sin \theta\left(s_{0}\right)=0, \quad$ and $\quad\left(\left(\kappa_{1}^{\prime \prime}\left(s_{0}\right)+\kappa_{1}\left(s_{0}\right) \kappa_{2}^{2}\left(s_{0}\right)\right) \cos \theta\left(s_{0}\right)\right.$ $\left.-2 \kappa_{1}^{\prime}\left(s_{0}\right) \kappa_{2}\left(s_{0}\right)-\kappa_{1}\left(s_{0}\right) \kappa_{2}^{\prime}\left(s_{0}\right)+\kappa_{1}\left(s_{0}\right) \kappa_{2}\left(s_{0}\right) \kappa_{3}\left(s_{0}\right) \sin \theta\left(s_{0}\right)\right)^{\prime}=0$.

The above proposition asserts that the discriminant set of the Lorentzian distance-squared function $G$ is given by

$$
\mathcal{D}_{H}=\mathbb{L}_{\mathbb{C}}\left(N_{1}(C)\left[\boldsymbol{n}^{T}\right] \times \mathbb{R}\right)=\left\{\lambda=\boldsymbol{\gamma}(s)-\mu \mathbb{N} G\left(\boldsymbol{n}^{T}\right)(s, \theta) \mid s \in I, \theta \in[0,2 \pi), \mu \in \mathbb{R}\right\} .
$$

Moreover, the lightlike focal surface is

$$
\mathbb{L} \mathbb{F}_{C}=\left\{\lambda=\gamma(s)-\frac{1}{\kappa_{1}(s) \cos \theta} \mathbb{N G}\left(\boldsymbol{n}^{T}\right)(s, \theta) \mid s \in I, \theta \in[0,2 \pi)\right\} .
$$

Here, we also denote that $\rho_{2}(s, \theta)=\kappa_{1}^{\prime}(s) \cos \theta-\kappa_{1}(s) \kappa_{2}(s)$ and

$$
\eta_{2}(s, \theta)=\left(\kappa_{1}^{\prime \prime}(s)+\kappa_{1}(s) \kappa_{2}^{2}(s)\right) \cos \theta-2 \kappa_{1}^{\prime}(s) \kappa_{2}(s)-\kappa_{1}(s) \kappa_{2}^{\prime}(s)+\kappa_{1}(s) \kappa_{2}(s) \kappa_{3}(s) \sin \theta .
$$

We can also show that $\rho_{2}(s, \theta)=\eta_{2}(s, \theta)=0$ if and only if $\rho_{2}(s, \theta)=\sigma_{2}(s)=0$, where

$$
\sigma_{2}(s)=\left[\kappa_{1} \kappa_{2}\left(\kappa_{1}^{\prime \prime}+\kappa_{1} \kappa_{2}^{2}\right)-\kappa_{1}^{\prime}\left(2 \kappa_{1}^{\prime} \kappa_{2}+\kappa_{1} \kappa_{2}^{\prime}\right) \pm \kappa_{1} \kappa_{2} \kappa_{3} \sqrt{-\left(\kappa_{1} \kappa_{2}\right)^{2}+\left(\kappa_{1}^{\prime}\right)^{2}}\right](s) .
$$

\section{Case 3}

Suppose that $\boldsymbol{n}_{3}(s)$ is timelike. Then, we adopt $\boldsymbol{n}^{T}(s)=\boldsymbol{n}_{3}(s)$ and denote that $\boldsymbol{b}_{1}(s)=\boldsymbol{n}_{1}(s), \boldsymbol{b}_{2}(s)$ $=\boldsymbol{n}_{2}(s)$. We have a pseudo-orthogonal frame $\left\{\boldsymbol{\gamma}(s), \boldsymbol{t}(s), \boldsymbol{n}^{T}(s), \boldsymbol{b}_{1}(s), \boldsymbol{b}_{2}(s)\right\}$ and $\delta_{3}=-1$ and $\delta_{1}=$ $\delta_{2}=1$, which satisfies the following Frenet-Serret type formulae:

$$
\left\{\begin{array}{l}
\boldsymbol{\gamma}^{\prime}(s)=\boldsymbol{t}(s), \\
\boldsymbol{t}^{\prime}(s)=\boldsymbol{\gamma}(s)+\kappa_{1}(s) \boldsymbol{b}_{1}(s), \\
\boldsymbol{b}_{1}^{\prime}(s)=-\kappa_{1}(s) \boldsymbol{t}(s)+\kappa_{2}(s) \boldsymbol{b}_{2}(s), \\
\boldsymbol{b}_{2}^{\prime}(s)=-\kappa_{2}(s) \boldsymbol{b}_{1}(s)+\kappa_{3}(s) \boldsymbol{n}^{T}(s), \\
\boldsymbol{n}^{T^{\prime}}(s)=\kappa_{3}(s) \boldsymbol{b}_{2}(s),
\end{array}\right.
$$

here, $N_{1}(C)\left[\boldsymbol{n}^{T}\right]$ is parametrized by

$$
N_{1}(C)\left[\boldsymbol{n}^{T}\right]=\left\{(\boldsymbol{\gamma}(s), \boldsymbol{\xi}) \in \boldsymbol{\gamma}^{*} T \mathbb{R}_{1}^{5} \mid \boldsymbol{\xi}=\cos \theta \boldsymbol{b}_{1}(s)+\sin \theta \boldsymbol{b}_{2}(s) \in N_{\gamma(s)}(C), s \in I\right\},
$$


so that we have the lightlike hypersurface along $C$,

$$
\mathbb{L} \mathbb{H}_{C}((s, \theta), t)=\gamma(s)+t \mathbb{N} \mathbb{G}\left(\boldsymbol{n}^{T}\right)(s, \theta) .
$$

We investigate the anti-de Sitter function on a spacelike curve $C=\gamma(I)$ By the calculations similar to the cases 1 and 2, we have the following proposition.

Proposition 7.3. Let $C$ be a spacelike curve and $H: C \times\left(A d S^{4} \backslash C\right) \rightarrow \mathbb{R}$ the anti-de Sitter function on $C=\gamma(I)$. Suppose that $p_{0} \neq \lambda_{0}$. Then, we have the following:

(1) $h\left(p_{0}\right)=h^{\prime}\left(p_{0}\right)=0$ if and only if there exist $\theta_{0} \in[0,2 \pi)$ and $\mu \in \mathbb{R} \backslash\{0\}$ such that

$$
\gamma\left(s_{0}\right)-\lambda_{0}=\mu \mathbb{N} \mathbb{G}\left(\boldsymbol{n}^{T}\right)\left(s_{0}, \theta_{0}\right) .
$$

(2) $\quad h\left(p_{0}\right)=h^{\prime}\left(p_{0}\right)=h^{\prime \prime}\left(p_{0}\right)=0$ if and only if there exists $\theta_{0} \in[0, s \pi)$ such that

$$
\boldsymbol{\gamma}\left(s_{0}\right)-\lambda_{0}=\frac{1}{\kappa_{1}\left(s_{0}\right) \cos \theta_{0}} \mathbb{N G}\left(\boldsymbol{n}^{T}\right)\left(s_{0}, \theta_{0}\right) .
$$

(3) $\quad h\left(p_{0}\right)=h^{\prime}\left(p_{0}\right)=h^{\prime \prime}\left(p_{0}\right)=h^{\prime \prime \prime}\left(p_{0}\right)=0$ if and only if there exists $\theta_{0} \in[0, s \pi)$ such that

$$
\boldsymbol{\gamma}\left(s_{0}\right)-\lambda_{0}=\frac{1}{\kappa_{1}\left(s_{0}\right) \cos \theta_{0}} \mathbb{N} \mathbb{G}\left(\boldsymbol{n}^{T}\right)\left(s_{0}, \theta_{0}\right)
$$

and $\kappa_{1}^{\prime}\left(s_{0}\right) \cos \theta_{0}+\kappa_{1}\left(s_{0}\right) \kappa_{2}\left(s_{0}\right) \sin \theta_{0}=0$, so that we can write $\theta_{0}=\theta\left(s_{0}\right)$.

(4) $\quad h\left(p_{0}\right)=h^{\prime}\left(p_{0}\right)=h^{\prime \prime}\left(p_{0}\right)=h^{\prime \prime \prime}\left(p_{0}\right)=h^{(4)}\left(p_{0}\right)=0$ if and only if there exists $\theta_{0}=\theta\left(s_{0}\right) \in[0,2 \pi)$ such that

$$
\boldsymbol{\gamma}\left(s_{0}\right)-\lambda_{0}=\frac{1}{\kappa_{1}\left(s_{0}\right) \cos \theta\left(s_{0}\right)} \mathbb{N G}\left(\boldsymbol{n}^{T}\right)\left(s_{0}, \theta\left(s_{0}\right)\right),
$$

$\kappa_{1}^{\prime}\left(s_{0}\right) \cos \theta\left(s_{0}\right)+\kappa_{1}\left(s_{0}\right) \kappa_{2}\left(s_{0}\right) \sin \theta\left(s_{0}\right)=0$ and $\left(2 \kappa_{1}^{\prime}\left(s_{0}\right) \kappa_{2}\left(s_{0}\right)+\kappa_{1}\left(s_{0}\right) \kappa_{2}^{\prime}\left(s_{0}\right)\right) \sin \theta\left(s_{0}\right)+\left(\kappa_{1}^{\prime \prime}\right.$ $\left.\left(s_{0}\right)-\kappa_{1}\left(s_{0}\right) \kappa_{2}^{2}\left(s_{0}\right)\right) \cos \theta\left(s_{0}\right)-\kappa_{1}\left(s_{0}\right) \kappa_{2}\left(s_{0}\right) \kappa_{3}\left(s_{0}\right)=0$.

(5) $\quad h\left(p_{0}\right)=h^{\prime}\left(p_{0}\right)=h^{\prime \prime}\left(p_{0}\right)=h^{\prime \prime \prime}\left(p_{0}\right)=h^{(4)}\left(p_{0}\right)=h^{(5)}\left(p_{0}\right)=0$ if and only if there exists $\theta_{0}=$ $\theta\left(s_{0}\right) \in[0,2 \pi)$ such that

$$
\boldsymbol{\gamma}\left(s_{0}\right)-\lambda_{0}=\frac{1}{\kappa_{1}\left(s_{0}\right) \cos \theta\left(s_{0}\right)} \mathbb{N} \mathbb{G}\left(\boldsymbol{n}^{T}\right)\left(s_{0}, \theta\left(s_{0}\right)\right),
$$

$\kappa_{1}^{\prime}\left(s_{0}\right) \cos \theta\left(s_{0}\right)+\kappa_{1}\left(s_{0}\right) \kappa_{2}\left(s_{0}\right) \sin \theta\left(s_{0}\right)=0$ and $\left(\left(2 \kappa_{1}^{\prime}\left(s_{0}\right) \kappa_{2}\left(s_{0}\right)+\kappa_{1}\left(s_{0}\right) \kappa_{2}^{\prime}\left(s_{0}\right) \sin \theta\left(s_{0}\right)\right)+\left(\kappa_{1}^{\prime \prime}\right.\right.$ $\left.\left.\left(s_{0}\right)-\kappa_{1}\left(s_{0}\right) \kappa_{2}^{2}\left(s_{0}\right)\right) \cos \theta\left(s_{0}\right)-\kappa_{1}\left(s_{0}\right) \kappa_{2}\left(s_{0}\right) \kappa_{3}\left(s_{0}\right)\right)^{\prime}=0$.

The above proposition asserts that the discriminant set of the anti-de Sitter function $H$ is given by

$$
\mathcal{D}_{H}=\mathbb{L} \mathbb{H}_{C}\left(N_{1}(C)\left[\boldsymbol{n}^{T}\right] \times \mathbb{R}\right)=\left\{\lambda=\boldsymbol{\gamma}(s)+t \mathbb{N} G\left(\boldsymbol{n}^{T}\right)(s, \theta) \mid s \in I, \theta \in[0,2 \pi), t \in \mathbb{R}\right\} .
$$

Moreover, the lightlike focal surface is

$$
\mathbb{L F}_{C}=\left\{\lambda=\gamma(s)-\frac{1}{\kappa_{1}(s) \cos \theta} \mathbb{N G}\left(\boldsymbol{n}^{T}\right)(s, \theta) \mid s \in I, \theta \in[0,2 \pi)\right\} .
$$

Here, we also denote that $\rho_{3}(s, \theta)=\kappa_{1}^{\prime}(s) \cos \theta+\kappa_{1}(s) \kappa_{2}(s) \sin \theta$ and

$$
\eta_{3}(s, \theta)=\left(2 \kappa_{1}^{\prime}(s) \kappa_{2}(s)+\kappa_{1}(s) \kappa_{2}^{\prime}(s)\right) \sin \theta+\left(\kappa_{1}^{\prime \prime}(s)-\kappa_{1}(s) \kappa_{2}^{2}(s)\right) \cos \theta-\kappa_{1}(s) \kappa_{2}(s) \kappa_{3}(s) .
$$

We can also show that $\rho_{3}(s, \theta)=\eta_{3}(s, \theta)=0$ if and only if $\rho_{3}(s, \theta)=\sigma_{3}(s)=0$, where

$$
\sigma_{3}(s)=\left[\kappa_{1} \kappa_{2}\left(\kappa_{1}^{\prime \prime}-\kappa_{1} \kappa_{2}^{2}\right)-\kappa_{1}^{\prime}\left(2 \kappa_{1}^{\prime} \kappa_{2}+\kappa_{1} \kappa_{2}^{\prime}\right) \mp \kappa_{1} \kappa_{2} \kappa_{3} \sqrt{\left(\kappa_{1} \kappa_{2}\right)^{2}+\left(\kappa_{1}^{\prime}\right)^{2}}\right](s) .
$$

We can unify the invariants $\sigma_{i}(s),(i=1,2,3)$ as follows:

$$
\sigma(s)=\left[\kappa_{1} \kappa_{2}\left(\kappa_{1}^{\prime \prime}-\kappa_{1} \kappa_{2}^{2}\right)-\kappa_{1}^{\prime}\left(2 \kappa_{1}^{\prime} \kappa_{2}+\kappa_{1} \kappa_{2}^{\prime}\right) \mp \delta_{2} \kappa_{1} \kappa_{2} \kappa_{3} \sqrt{\delta_{1}\left(\kappa_{1} \kappa_{2}\right)^{2}+\delta_{2}\left(\kappa_{1}^{\prime}\right)^{2}}\right](s) .
$$

\section{Classifications of singularities}

By using the results of the three cases, we classify the singularities of the lightlike hypersurface along $\gamma$ as an application of the unfolding theory of functions. For a function $f(s)$, we say that $f$ 
has $A_{k}$-singularity at $s_{0}$ if $f^{(p)}\left(s_{0}\right)=0$ for all $1 \leq p \leq k$ and $f^{(k+1)}\left(s_{0}\right) \neq 0$. Let $F$ be an $r$-parameter unfolding of $f$ and $f$ has $A_{k}$-singularity $(k \geq 1)$ at $s_{0}$. We denote the $(k-1)$-jet of the partial derivative $\partial F / \partial x_{i}$ at $s_{0}$ as

$$
j^{(k-1)}\left(\frac{\partial F}{\partial x_{i}}\left(s, x_{0}\right)\right)\left(s_{0}\right)=\sum_{j=1}^{k-1} \alpha_{j i}\left(s-s_{0}\right)^{j},(i=1, \ldots, r) .
$$

If the rank of $k \times r$ matrix $\left(\alpha_{0 i}, \alpha_{j i}\right)$ is $k(k \leq r)$, then $F$ is called a versal unfolding of $f$, where $\alpha_{0 i}=\partial F / \partial x_{i}\left(s_{0}, \boldsymbol{x}_{0}\right)$.

Inspired by the propositions in Subsections VII A-VII C, we define the following set:

$$
D_{F}^{\ell}=\left\{\boldsymbol{x} \in \mathbb{R}^{r} \mid \exists s \in \mathbb{R}, F(s, \boldsymbol{x})=\frac{\partial F}{\partial s}(s, \boldsymbol{x})=\cdots=\frac{\partial^{\ell} F}{\partial s^{\ell}}(s, \boldsymbol{x})=0\right\},
$$

which is called a discriminant set of order $\ell$. Of course, $D_{F}^{1}=D_{F}$ and $D_{F}^{2}$ is the set of singular points of $D_{F}$. Therefore, we have the following proposition.

\section{Proposition 7.4. For all the cases, we have}

$$
D_{G}=D_{G}^{1}=\mathbb{L}_{C}\left(N_{1}(C)\left[\boldsymbol{n}^{T}\right] \times \mathbb{R}\right), D_{G}^{2}=\mathbb{L F}_{C} \text { and } D_{G}^{3} \text { is the critical value set of } \mathbb{L F}_{C} \text {. }
$$

In order to understand the geometric properties of the discriminant set of order $\ell$, we introduce an equivalence relation among the unfoldings of functions. Let $F$ and $G$ be $r$-parameter unfoldings of $f(s)$ and $g(s)$, respectively. We say that $F$ and $G$ are $P$-R-equivalent if there exists a diffeomorphism germ $\Phi:\left(\mathbb{R} \times \mathbb{R}^{r},\left(s_{0}, \boldsymbol{x}_{0}\right)\right) \longrightarrow\left(\mathbb{R} \times \mathbb{R}^{r},\left(s_{0}^{\prime}, \boldsymbol{x}_{0}^{\prime}\right)\right)$ of the form $\Phi(s, \boldsymbol{x})=\left(\Phi_{1}(s, \boldsymbol{x}), \phi(\boldsymbol{x})\right)$ such that $G \circ \Phi=F$. By straightforward calculations, we have the following proposition.

Proposition 7.5. Let $F$ and $G$ be r-parameter unfoldings of $f(s)$ and $g(s)$, respectively. If $F$ and $G$ are $P$-R-equivalent by a diffeomorphism germ $\Phi:\left(\mathbb{R} \times \mathbb{R}^{r},\left(s_{0}, \boldsymbol{x}_{0}\right)\right) \longrightarrow\left(\mathbb{R} \times \mathbb{R}^{r},\left(s_{0}^{\prime}, \boldsymbol{x}_{0}^{\prime}\right)\right)$ of the form $\Phi(s, \boldsymbol{x})=\left(\Phi_{1}(s, \boldsymbol{x}), \phi(\boldsymbol{x})\right)$, then $\phi\left(D_{F}^{\ell}\right)=D_{G}^{\ell}$ as set germs.

We have the following classification theorem of versal unfoldings [Ref. 3, p. 149, 6.6].

Theorem 7.6. Let $F:\left(\mathbb{R} \times \mathbb{R}^{r},\left(s_{0}, \boldsymbol{x}_{0}\right)\right) \longrightarrow \mathbb{R}$ be an r-parameter unfolding of $f$ which has $A_{k}$-singularity at $s_{0}$. Suppose $F$ is a versal unfolding of $f$, then $F$ is $P$-R-equivalent to one of the following unfoldings:

(a) $k=1 ; \pm s^{2}+x_{1}$

(b) $k=2 ; s^{3}+x_{1}+s x_{2}$,

(c) $k=3 ; \pm s^{4}+x_{1}+s x_{2}+s^{2} x_{3}$,

(d) $k=4 ; s^{5}+x_{1}+s x_{2}+s^{2} x_{3}+s^{3} x_{4}$.

We have the following classification result as a corollary of the above theorem.

Corollary 7.7. Let $F:\left(\mathbb{R} \times \mathbb{R}^{r},\left(s_{0}, \boldsymbol{x}_{0}\right)\right) \longrightarrow \mathbb{R}$ be an r-parameter unfolding of $f$ which has $A_{k}$-singularity at $s_{0}$. Suppose $F$ is a versal unfolding of $f$, then we have the following assertions:

(a) If $k=1$, then $D_{F}$ is diffeomorphic to $\{0\} \times \mathbb{R}^{r-1}$ and $D_{F}^{2}=\emptyset$.

(b) If $k=2$, then $D_{F}$ is diffeomorphic to $C(2,3) \times \mathbb{R}^{r-2}, D_{F}^{2}$ is diffeomorphic to $\{\mathbf{0}\} \times \mathbb{R}^{r-2}$, and $D_{F}^{3}=\emptyset$.

(c) If $k=3$, then $D_{F}$ is diffeomorphic to $S W \times \mathbb{R}^{r-3}, D_{F}^{2}$ is diffeomorphic to $C(2,3,4) \times \mathbb{R}^{r-3}, D_{F}^{3}$ is diffeomorphic to $\{\mathbf{0}\} \times \mathbb{R}^{r-3}$, and $D_{F}^{4}=\emptyset$.

(d) If $k=4$, then $D_{F}$ is locally diffeomorphic to $B F \times \mathbb{R}^{r-4}, D_{F}^{2}$ is diffeomorphic to $C(B F) \times$ $\mathbb{R}^{r-4}, D_{F}^{3}$ is diffeomorphic to $C(2,3,4,5) \times \mathbb{R}^{r-4}, D_{F}^{4}$ is diffeomorphic to $\{\boldsymbol{0}\} \times \mathbb{R}^{r-4}$, and $D_{F}^{5}=\emptyset$.

We remark that all of diffeomorphisms in the above assertions are diffeomorphism germs.

Here, we call $B F=\left\{\left(x_{1}, x_{2}, x_{3} . x_{4}\right) \mid x_{1}=5 u^{4}+3 v u^{2}+2 w u, x_{2}=4 u^{5}+2 v u^{3}+w u^{2}, x_{3}=u, x_{4}\right.$ $=v\}$ a butterfly. We have the following key proposition on $H$. 
Proposition 7.8. If $h(s)$ has $A_{k}$-singularity $(k=1,2,3,4)$ at $p_{0}$, then $H$ is a versal unfolding of $h$.

Proof. We denote that $\gamma(s)=\left(X_{-1}(s), X_{0}(s), X_{1}(s), X_{2}(s), X_{3}(s)\right)$ and $\lambda=\left(\lambda_{-1}, \lambda_{0}, \lambda_{1}, \lambda_{2}, \lambda_{3}\right)$.

By definition, we have

$$
H(s, \lambda)=-\lambda_{-1} X_{-1}(s)-\lambda_{0} X_{0}(s)+\lambda_{1} X_{1}(s)+\lambda_{2} X_{2}(s)+\lambda_{3} X_{3}(s)+1 .
$$

Thus, we have

$$
\begin{gathered}
\frac{\partial H}{\partial \lambda_{i}}(s, \lambda)=-X_{i}(s) \text { and } \frac{\partial^{2} H}{\partial s \partial \lambda_{i}}(s, \lambda)=-X_{i}^{\prime}(s), \text { for }(i=-1,0), \\
\frac{\partial H}{\partial \lambda_{i}}(s, \lambda)=X_{i}(s) \text { and } \frac{\partial^{2} H}{\partial s \partial \lambda_{i}}(s, \lambda)=X_{i}^{\prime}(s), \text { for }(i=1,2,3) .
\end{gathered}
$$

For a fixed $\lambda_{0}=\left(\lambda_{0-1} \lambda_{00}, \lambda_{01}, \lambda_{02}, \lambda_{03}\right)$, the 3-jet of $\partial H / \partial \lambda_{i}\left(s, \lambda_{0}\right)(i=-1,0,1,2,3)$ at $s_{0}$ is

$$
\begin{aligned}
& j^{(3)} \frac{\partial H}{\partial \lambda_{i}}\left(s, \lambda_{0}\right)\left(s_{0}\right)=-X_{i}^{\prime}\left(s_{0}\right)\left(s-s_{0}\right)-\frac{1}{2} X_{i}^{\prime \prime}\left(s_{0}\right)\left(s-s_{0}\right)^{2}-\frac{1}{3} X_{i}^{\prime \prime \prime}\left(s_{0}\right)\left(s-s_{0}\right)^{3}(i=-1,0), \\
& j^{(3)} \frac{\partial H}{\partial \lambda_{i}}\left(s, \lambda_{0}\right)\left(s_{0}\right)=X_{i}^{\prime}\left(s_{0}\right)\left(s-s_{0}\right)+\frac{1}{2} X_{i}^{\prime \prime}\left(s_{0}\right)\left(s-s_{0}\right)^{2}+\frac{1}{3} X_{i}^{\prime \prime \prime}\left(s_{0}\right)\left(s-s_{0}\right)^{3}(i=1,2,3) .
\end{aligned}
$$

It is enough to show that the rank of the following matrix $\mathrm{A}$ is four:

$$
A=\left(\begin{array}{lllll}
-X_{-1}\left(s_{0}\right) & -X_{0}\left(s_{0}\right) & X_{1}\left(s_{0}\right) & X_{2}\left(s_{0}\right) & X_{3}\left(s_{0}\right) \\
-X_{-1}^{\prime}\left(s_{0}\right) & -X_{0}^{\prime}\left(s_{0}\right) & X_{1}^{\prime}\left(s_{0}\right) & X_{2}^{\prime}\left(s_{0}\right) & X_{3}^{\prime}\left(s_{0}\right) \\
-X_{-1}^{\prime \prime}\left(s_{0}\right) & -X_{0}^{\prime \prime}\left(s_{0}\right) & X_{1}^{\prime \prime}\left(s_{0}\right) & X_{2}^{\prime \prime}\left(s_{0}\right) & X_{3}^{\prime \prime}\left(s_{0}\right) \\
-X_{-1}^{\prime \prime \prime}\left(s_{0}\right) & -X_{0}^{\prime \prime \prime}\left(s_{0}\right) & X_{1}^{\prime \prime \prime}\left(s_{0}\right) & X_{2}^{\prime \prime \prime}\left(s_{0}\right) & X_{3}^{\prime \prime \prime}\left(s_{0}\right)
\end{array}\right) .
$$

Here, we consider the following matrix B:

$$
B=\left(\begin{array}{ccccc}
-X_{-1}\left(s_{0}\right) & -X_{0}\left(s_{0}\right) & X_{1}\left(s_{0}\right) & X_{2}\left(s_{0}\right) & X_{3}\left(s_{0}\right) \\
-X_{-1}^{\prime}\left(s_{0}\right) & -X_{0}^{\prime}\left(s_{0}\right) & X_{1}^{\prime}\left(s_{0}\right) & X_{2}^{\prime}\left(s_{0}\right) & X_{3}^{\prime}\left(s_{0}\right) \\
-X_{-1}^{\prime \prime}\left(s_{0}\right) & -X_{0}^{\prime \prime}\left(s_{0}\right) & X_{1}^{\prime \prime}\left(s_{0}\right) & X_{2}^{\prime \prime}\left(s_{0}\right) & X_{3}^{\prime \prime}\left(s_{0}\right) \\
-X_{-1}^{\prime \prime}\left(s_{0}\right) & -X_{0}^{\prime \prime \prime}\left(s_{0}\right) & X_{1}^{\prime \prime \prime}\left(s_{0}\right) & X_{2}^{\prime \prime \prime}\left(s_{0}\right) & X_{3}^{\prime \prime \prime}\left(s_{0}\right) \\
-n_{-1}\left(s_{0}\right) & -n_{0}\left(s_{0}\right) & n_{1}\left(s_{0}\right) & n_{2}\left(s_{0}\right) & n_{3}\left(s_{0}\right)
\end{array}\right),
$$

where we denote $\boldsymbol{n}_{1}\left(s_{0}\right)=\left(n_{-1}\left(s_{0}\right), n_{0}\left(s_{0}\right), n_{1}\left(s_{0}\right), n_{2}\left(s_{0}\right), n_{3}\left(s_{0}\right)\right)$. In fact,

$$
\begin{aligned}
\operatorname{det} B & =\operatorname{det}^{t}\left(\boldsymbol{\gamma}(s), \boldsymbol{\gamma}^{\prime}(s), \boldsymbol{\gamma}^{\prime \prime}(s), \boldsymbol{\gamma}^{\prime \prime \prime}(s), \boldsymbol{n}_{1}\right) \\
& =\operatorname{det}^{t}\left(\boldsymbol{\gamma}(s), \boldsymbol{\gamma}^{\prime}(s), \boldsymbol{\gamma}^{\prime \prime}(s)-\boldsymbol{\gamma}(s), \boldsymbol{b}, \boldsymbol{n}_{1}\right) \\
& =\operatorname{det}^{t}\left(\boldsymbol{\gamma}(s), \boldsymbol{\gamma}^{\prime}(s), \boldsymbol{\gamma}^{\prime \prime}(s)-\boldsymbol{\gamma}(s)-\kappa_{1} \boldsymbol{n}_{1}, \boldsymbol{b}-\kappa_{1} \boldsymbol{n}_{1}, \boldsymbol{n}_{1}\right),
\end{aligned}
$$

where $\boldsymbol{b}=\boldsymbol{\gamma}^{\prime \prime \prime}(s)+\frac{\kappa_{1}^{\prime}(s)}{\kappa_{1}(s)} \gamma(s)+\left(\delta_{1} \kappa_{1}^{2}(s)-1\right) \boldsymbol{\gamma}^{\prime}(s)+\left(1-\frac{\kappa_{1}^{\prime}(s)}{\kappa_{1}}\right) \boldsymbol{\gamma}^{\prime \prime}(s)$, and $\boldsymbol{\gamma}(s), \boldsymbol{\gamma}^{\prime}(s), \boldsymbol{\gamma}^{\prime \prime}(s)-\gamma(s)-$ $\kappa_{1} \boldsymbol{n}_{1}, \boldsymbol{b}-\kappa_{1} \boldsymbol{n}_{1}$, and $\boldsymbol{n}_{1}$ are linearly independent of each other in all cases 1,2,3, respectively. Therefore, we have $\operatorname{det} \mathrm{B} \neq 0$. This means that rankA $=4$. This completes the proof.

Finally, we can apply Corollary 8.5 to our condition. Then, we have the following theorem.

Theorem 7.9. Let $\gamma: I \longrightarrow$ AdS $S^{4}$ be a spacelike curves with $\kappa_{1}(s) \neq 0$.

(A) For the lightlike hypersurfaces $\mathbb{L}_{\mathbb{C}}((s, \theta), t)$ of $C=\gamma(I)$ in case 1 , we have the following assertions:

(1) The lightlike hypersurface $\mathbb{L H}_{C}\left(N_{1}(C)\left[\boldsymbol{n}^{T}\right] \times \mathbb{R}\right)$ is locally diffeomorphic to $C(2,3) \times$ $\mathbb{R}^{2}$ at $\lambda_{0}$ if and only if there exist $\theta_{0} \in[0,2 \pi)$ such that

$$
p_{0}-\lambda_{0}=-\frac{1}{\kappa_{1}\left(s_{0}\right)} \mathbb{N G}\left(\boldsymbol{n}^{T}\right)\left(s_{0}, \theta_{0}\right),
$$

and $\rho_{1}\left(s_{0}, \theta_{0}\right) \neq 0$. In this case, the lightlike focal set $\mathbb{L} \mathbb{F}_{C}$ is non-singular. 
(2) The lightlike hypersurface $\mathbb{L}_{C}\left(N_{1}(C)\left[\boldsymbol{n}^{T}\right] \times \mathbb{R}\right)$ is locally diffeomorphic to $S W \times \mathbb{R}$ at $\lambda_{0}$ if and only if there exist $\theta_{0} \in[0,2 \pi)$ such that

$$
p_{0}-\lambda_{0}=-\frac{1}{\kappa_{1}\left(s_{0}\right)} \mathbb{N} G\left(\boldsymbol{n}^{T}\right)\left(s_{0}, \theta_{0}\right),
$$

$\rho_{1}\left(s_{0}, \theta_{0}\right)=0$ and $\sigma_{1}\left(s_{0}\right) \neq 0$. In this case, the lightlike focal set $\mathbb{L F}_{C}$ is locally diffeomorphic to $C(2,3,4) \times \mathbb{R}$ and the critical value set of $\mathbb{L} \mathbb{F}_{C}$ is a regular curve.

(3) The lightlike hypersurface $\mathbb{L} \mathbb{H}_{C}\left(N_{1}(C)\left[\boldsymbol{n}^{T}\right] \times \mathbb{R}\right)$ is locally diffeomorphic to $B F$ at $\lambda_{0}$ if and only if there exist $\theta_{0} \in[0,2 \pi)$ such that

$$
p_{0}-\lambda_{0}=-\frac{1}{\kappa_{1}\left(s_{0}\right)} \mathbb{N G}\left(\boldsymbol{n}^{T}\right)\left(s_{0}, \theta_{0}\right),
$$

$\rho_{1}\left(s_{0}, \theta_{0}\right)=0, \sigma_{1}\left(s_{0}\right)=0$, and $\sigma_{1}^{\prime}\left(s_{0}\right) \neq 0$. In this case, the lightlike focal set $\mathbb{L}_{C}$ is locally diffeomorphic to $C(B F) \times \mathbb{R}$ and the critical value set is locally diffeomorphic to the $C(2,3,4,5)$-cusp.

(B) For the lightlike hypersurfaces $\mathbb{L} \mathbb{H}_{C}\left(N_{1}(C)\left[\boldsymbol{n}^{T}\right] \times \mathbb{R}\right)$ of $C=\gamma(I)$ in case 2 , we have the following assertions:

(1) The lightlike hypersurface $\mathbb{L}_{\mathbb{H}}\left(N_{1}(C)\left[\boldsymbol{n}^{T}\right] \times \mathbb{R}\right)$ is locally diffeomorphic to $C(2,3) \times$ $\mathbb{R}^{2}$ at $\lambda_{0}$ if and only if there exist $\theta_{0} \in[0,2 \pi)$ such that

$$
p_{0}-\lambda_{0}=\frac{1}{\kappa_{1}\left(s_{0}\right) \cos \theta_{0}} \mathbb{N G}\left(\boldsymbol{n}^{T}\right)\left(s_{0}, \theta_{0}\right)
$$

and $\rho_{2}\left(s_{0}, \theta_{0}\right) \neq 0$. In this case, the lightlike focal set $\mathbb{L} \mathbb{F}_{C}$ is non-singular.

(2) The lightlike hypersurface $\mathbb{L}_{\mathbb{C}}\left(N_{1}(C)\left[\boldsymbol{n}^{T}\right] \times \mathbb{R}\right)$ is locally diffeomorphic to $S W \times \mathbb{R}$ at $\lambda_{0}$ if and only if there exist $\theta_{0} \in[0,2 \pi)$ such that

$$
p_{0}-\lambda_{0}=\frac{1}{\kappa_{1}\left(s_{0}\right) \cos \theta_{0}} \mathbb{N} G\left(\boldsymbol{n}^{T}\right)\left(s_{0}, \theta_{0}\right),
$$

$\rho_{2}\left(s_{0}, \theta_{0}\right)=0$ and $\sigma\left(s_{0}\right) \neq 0$. In this case, the lightlike focal set $\mathbb{L F}_{C}$ is locally diffeomorphic to $C(2,3,4) \times \mathbb{R}$ and the critical value set of $\mathbb{L}_{C}$ is a regular curve.

(3) The lightlike hypersurface $\mathbb{L} \mathbb{H} C\left(N_{1}(C)\left[\boldsymbol{n}^{T}\right] \times \mathbb{R}\right)$ is locally diffeomorphic to $B F$ at $\lambda_{0}$ if and only if there exist $\theta_{0} \in[0,2 \pi)$ such that

$$
p_{0}-\lambda_{0}=\frac{1}{\kappa_{1}\left(s_{0}\right) \cos \theta_{0}} \mathbb{N} G\left(\boldsymbol{n}^{T}\right)\left(s_{0}, \theta_{0}\right),
$$

$\rho_{2}\left(s_{0}, \theta_{0}\right)=0, \sigma_{2}\left(s_{0}\right)=0$, and $\sigma_{2}^{\prime}\left(s_{0}\right) \neq 0$. In this case, the lightlike focal set $\mathbb{L F}_{C}$ is locally diffeomorphic to $C(B F) \times \mathbb{R}$ and the critical value set of $\mathbb{L F}_{C}$ is locally diffeomorphic to the $C(2,3,4,5)$-cusp.

(C) For the lightlike hypersurfaces $\mathbb{L} \mathbb{H}_{C}\left(N_{1}(C)\left[n^{T}\right] \times \mathbb{R}\right)$ of $C=\gamma(I)$ in case 3 , we have the following assertions:

(1) The lightlike hypersurface $\mathbb{L} \mathbb{H}_{C}\left(N_{1}(C)\left[\boldsymbol{n}^{T}\right] \times \mathbb{R}\right)$ is locally diffeomorphic to $C(2,3) \times$ $\mathbb{R}^{2}$ at $\lambda_{0}$ if and only if there exist $\theta_{0} \in[0,2 \pi)$ such that

$$
p_{0}-\lambda_{0}=\frac{1}{\kappa_{1}\left(s_{0}\right) \cos \theta_{0}} \mathbb{N} G\left(\boldsymbol{n}^{T}\right)\left(s_{0}, \theta_{0}\right),
$$

and $\rho_{3}\left(s_{0}, \theta_{0}\right) \neq 0$. In this case, the lightlike focal set $\mathbb{L F}_{C}$ is non-singular.

(2) The lightlike hypersurface $\mathbb{L}_{\mathbb{H}}\left(N_{1}(C)\left[\boldsymbol{n}^{T}\right] \times \mathbb{R}\right)$ is locally diffeomorphic to $S W \times \mathbb{R}$ at $\lambda_{0}$ if and only if there exist $\theta_{0} \in[0,2 \pi)$ such that

$$
p_{0}-\lambda_{0}=\frac{1}{\kappa_{1}\left(s_{0}\right) \cos \theta_{0}} \mathbb{N G}\left(\boldsymbol{n}^{T}\right)\left(s_{0}, \theta_{0}\right),
$$

$\rho_{3}\left(s_{0}, \theta_{0}\right)=0$ and $\sigma_{3}\left(s_{0}\right) \neq 0$. In this case, the lightlike focal set $\mathbb{L F}_{C}$ is locally diffeomorphic to $C(2,3,4) \times \mathbb{R}$ and the critical value set of $\mathbb{L F}_{C}$ is a regular curve.

(3) The lightlike hypersurface $\mathbb{L} \mathbb{H} C\left(N_{1}(C)\left[\boldsymbol{n}^{T}\right] \times \mathbb{R}\right)$ is locally diffeomorphic to $B F$ at $\lambda_{0}$ if and only if there exist $\theta_{0} \in[0,2 \pi)$ such that

$$
p_{0}-\lambda_{0}=\frac{1}{\kappa_{1}\left(s_{0}\right) \cos \theta_{0}} \mathbb{N G}\left(\boldsymbol{n}^{T}\right)\left(s_{0}, \theta_{0}\right),
$$


$\rho_{3}\left(s_{0}, \theta_{0}\right)=0, \sigma_{3}\left(s_{0}\right)=0$, and $\sigma_{3}^{\prime}\left(s_{0}\right) \neq 0$. In this case, the lightlike focal set $\mathbb{L F}_{C}$ is locally diffeomorphic to $C(B F) \times \mathbb{R}$ and the critical value set of $\mathbb{L F}_{C}$ is locally diffeomorphic to the $C(2,3,4,5)$-cusp.

${ }^{1}$ Arnol'd, V. I., Gusein-Zade, S. M., and Varchenko, A. N., Singularities of Differentiable Maps (Birkhäuser, 1986), Vol. I.

${ }^{2}$ Bouuso, R. and Randall, L., "Holographic domains of anti-de Sitter space," J. High Energy Phys. 04, 057 (2002).

${ }^{3}$ Bruce, J. W. and Giblin, P. J., Curves and Singularities, 2nd ed. (Cambridge University Press, 1992).

${ }^{4}$ Chandrasekhar, S., The Mathematical Theory of Black Holes, International Series of Monographs on Physics Vol. 69 (Oxford University Press, 1983).

${ }^{5}$ Chen, L., Han, Q., Pei, D.-H., and Sun, W., "The singularities of null surfaces in Anti de Sitter 3-space," J. Math. Anal. Appl. 366, 256-265 (2010).

${ }^{6}$ Gibson, C. G., Wirthmüller, K., du Plessis, A. A., and Looijenga, E. J., Topological Stability of Smooth Mappings, Lecture Notes in Mathematics Vol. 552 (Springer, Berlin, 1976).

${ }^{7}$ Izumiya, S., in The Second Japanese-Australian Workshop on Real and Complex Singularities ["Lightlike hypersurfaces in Lorentz-Minkowski space,” RIMS Kokyuroku 1610, 51-65 (2008)].

${ }^{8}$ Izumiya, S., Kossowski, M., Pei, D.-H., and Romero Fuster, M. C., "Singularities of lightlike hypersurfaces in Minkowski 4-space," Tohoku Math. J. 58, 71-88 (2006).

${ }^{9}$ Izumiya, S., Nunño Ballesteros, J. J., and Romero Fuster, M. C., "Global properties of codimension two spacelike submanifolds in Minkowski space," Adv. Geom. 10, 51-75 (2010).

${ }^{10}$ Izumiya, S., Pei, D.-H., and Romero Fuster, M. C., "The lightcone Gauss map of a spacelike surfaces in Minkowski 4-space," Asian J. Math. 8, 511-530 (2004)

${ }^{11}$ Izumiya, S., Pei, D.-H., and Romero Fuster, M. C., "Spacelike surfaces in Anti de Sitter four-space from a contact viewpoint," Proc. Steklov Math. Inst. 267, 156-173 (2009).

12 Izumiya, S., Pei, D.-H., Romero Fuster, M. C., and Takahashi, M., "The horospherical geometry of submanifolds in hyperbolic space," J. London Math. Soc. 71, 779-800 (2005).

${ }^{13}$ Izumiya, S., Pei, D.-H., and Sano, T., "Singularities of hyperbolic Gauss maps," Proc. London Math. Soc., 3 86, 485-512 (2003).

${ }^{14}$ Izumiya, S., Pei, D.-H., and Takahashi, M., "Singularities of evolutes of hypersurfaces in Hyperbolic space,” Proc. Edinburgh Math. Soc. 47, 131-153 (2004).

${ }^{15}$ Izumiya, S. and Romero-Fuster, M. C., “The horospherical Gauss-Bonnet type theorem in hyperbolic space,” J. Math. Soc. Jpn. 58, 965-984 (2006).

${ }^{16}$ Izumiya, S. and Romero Fuster, M. C., "The lightlike flat geometry on spacelike submanifolds of codimension two in Minkowski space," Selecta Math. 13(1), 23-55 (2007).

${ }^{17}$ Karch, A. and Randall, L., "Locally localized gravity," J. High Energy Phys. 05, 008 (2001)

${ }^{18}$ Kasedou, M., "Spacelike submanifolds of codimension two in de Sitter space," J. Geom. Phys. 60, 31-42 (2010).

${ }^{19}$ Kossowski, M., "The $S^{2}$-valued Gauss maps and split total curvature of space-like codimension-2 surface in Minkowski space,” J. London Math. Soc, 2 40, 179-192 (1989).

${ }^{20}$ Kossowski, M., "The intrinsic conformal structure and Gauss map of a light-like hypersurface in Minkowski space," Trans Am. Math. Soc. 316(1), 369-383 (1989).

${ }^{21}$ Little, J. A., "On singularities of submanifolds of high dimensional Euclidean space," Ann. Mat. Pura Appl, 4 83, 261-335 (1969).

22 Maldacena, M., "The large N limit of superconformal field theories and supergravity," Adv. Theor. Math. Phys. 2, 231-252 (1998).

${ }^{23}$ Martinet, J., Singularities of Smooth Functions and Maps, London Math. Soc. Lecture Note Series Vol. 58 (Cambridge University Press, 1982).

${ }^{24}$ Mather, J. N., "How to stratify mappings and jet spaces," in Singularités d'applications différentiables, (Sém., Plans-sur-Bex 1975), Lecture Notes in Mathematics Vol. 535 (Springer, Berlin, 1976), pp. 128-176.

${ }^{25}$ Misner, C. W., Thorne, K. S., and Wheeler, J. W., Gravitation (W. H. Freeman and Co., San Francisco, CA, 1973).

${ }^{26}$ Montaldi, J. A., "On contact between submanifolds,” Michigan Math. J. 33, 195-199 (1986).

27 O’Neill, B., Semi-Riemannian Geometry (Academic Press, New York, 1983).

${ }^{28}$ Randall, L. and Sundrum, R., "An alternative to compactification,” Phys. Rev. Lett. 83, 4690-4693 (1999).

${ }^{29}$ Wan, Y.-H., "Generic deformation of varieties," Trans. Am. Mat. Soc. 259, 107-119 (1980).

${ }^{30}$ Wassermann, G., "Stability of Caustics," Math. Ann. 216, 43-50 (1975).

${ }^{31}$ Witten, E., "Anti de Sitter space and holography," Adv. Theor. Math. Phys. 2, 253-291 (1998).

32 Zakalyukin, V. M., "Lagrangian and Legendrian singularities," Funct. Anal. Appl. 10, 23-31 (1976). 